\title{
Tandem Photoisomerization \& Transannular Cyclizations of Zerumbone Epoxide: A Model for Diversity-Oriented Synthesis using Abundant Natural Products
}

Mohanan Biji ${ }^{\S, \omega}$ Kokkuvayil Vasu Radhakrishnan, ${ }^{\S, \omega, *}$ and Ravi S. Lankalapalli, ${ }^{\S, \omega, *}$

${ }^{\S}$ Chemical Sciences and Technology Division, CSIR-National Institute for Interdisciplinary Science and Technology, Thiruvananthapuram, 695 019, India; and ${ }^{\omega}$ Academy of Scientific \& Innovative Research (AcSIR), Ghaziabad-201002, India.

*Corresponding Authors: radhu2005@gmail.com, ravishankar@niist.res.in

\section{Supporting Information}

\section{Table of contents}

\begin{tabular}{|c|c|c|}
\hline 1 & General experimental conditions & S3 \\
\hline 2 & $\begin{array}{l}\text { Scheme S1. Geometrical isomers of zerumbone epoxide } 1 \text { that afford } \\
\text { compound } 3 \text { with cis-ring junction }\end{array}$ & S6 \\
\hline 3 & $\begin{array}{l}\text { Scheme S2. Photoirradiation attempt made on 10a, a functionalized } \\
\text { C13(OAc) of zerumbone epoxide } \mathbf{1}\end{array}$ & S7 \\
\hline 4 & $\begin{array}{l}\text { Table S1. }{ }^{1} \mathrm{H}(500 \mathrm{MHz}),{ }^{13} \mathrm{C}(125 \mathrm{MHz}) ; \mathrm{COSY} \text { and HMBC NMR data of } \\
\text { compound } 2 \text { in acetone-d6. }\end{array}$ & S8 \\
\hline 5 & Figure S1. 1D NOE spectra of compound 2 & S10 \\
\hline 6 & Figure S2. HRMS of compound 2 & S10 \\
\hline 7 & $\begin{array}{l}\text { Table S2. }{ }^{1} \mathrm{H}(500 \mathrm{MHz}),{ }^{13} \mathrm{C}(125 \mathrm{MHz}) \text {; COSY and HMBC NMR data of } \\
\text { compound } 3 \text { in } \mathrm{CDCl}_{3} \text {. }\end{array}$ & S11 \\
\hline 8 & Figure S3. HRMS of compound $\mathbf{3}$ & S12 \\
\hline 9 & $\begin{array}{l}\text { Table S3. }{ }^{1} \mathrm{H}(500 \mathrm{MHz}),{ }^{13} \mathrm{C}(125 \mathrm{MHz}) \text {; COSY and HMBC NMR data of } \\
\text { compound } 4 \text { in } \mathrm{CDCl}_{3 .} \text {. }\end{array}$ & S13 \\
\hline 10 & Figure S4. HRMS of compound 4 & S14 \\
\hline 11 & Figure S5. ${ }^{1} \mathrm{H}$ NMR spectra of different isomers of zerumbone-2,3-epoxide & S15 \\
\hline 12 & $\begin{array}{l}\text { Table S4. }{ }^{1} \mathrm{H}(500 \mathrm{MHz}),{ }^{13} \mathrm{C}(125 \mathrm{MHz}) ; \mathrm{COSY} \text { and HMBC NMR data of } \\
\text { compound } \mathbf{5 a} \text { in } \mathrm{CDCl}_{3} \text {. }\end{array}$ & S16 \\
\hline 13 & Figure S6. HRMS of compound $\mathbf{5 a}$ & S18 \\
\hline 14 & Figure S7. 1D NOE spectrum of compound $\mathbf{5 a}$ & S18 \\
\hline
\end{tabular}




\begin{tabular}{|c|c|c|}
\hline 15 & $\begin{array}{l}\text { Table S5. }{ }^{1} \mathrm{H}(500 \mathrm{MHz}),{ }^{13} \mathrm{C}(125 \mathrm{MHz}) ; \mathrm{COSY} \text { and } \mathrm{HMBC} \text { NMR data of } \\
\text { compound } \mathbf{5 b} \text { in } \mathrm{CDCl}_{3} \text {. }\end{array}$ & S19 \\
\hline 16 & Figure S8. HRMS of compound $\mathbf{5 b}$ & S20 \\
\hline 17 & $\begin{array}{l}\text { Table S6. }{ }^{1} \mathrm{H}(500 \mathrm{MHz}),{ }^{13} \mathrm{C}(125 \mathrm{MHz}) \text {; COSY and HMBC NMR data of } \\
\text { compound } \mathbf{5 c} \text { in } \mathrm{CDCl}_{3} \text {. }\end{array}$ & S21 \\
\hline 18 & Figure S9. HRMS of compound $\mathbf{5 c}$ & S22 \\
\hline 19 & $\begin{array}{l}\text { Table S7. }{ }^{1} \mathrm{H}(500 \mathrm{MHz}),{ }^{13} \mathrm{C}(125 \mathrm{MHz}) ; \mathrm{COSY} \text { and } \mathrm{HMBC} \text { NMR data of } \\
\text { compound } \mathbf{6 a} \text { in } \mathrm{CDCl}_{3} \text {. }\end{array}$ & S23 \\
\hline 20 & Figure S10. HRMS of compound $\mathbf{6 a}$ & S24 \\
\hline 21 & $\begin{array}{l}\text { Table S8. }{ }^{1} \mathrm{H}(500 \mathrm{MHz}),{ }^{13} \mathrm{C}(125 \mathrm{MHz}) ; \mathrm{COSY} \text { and } \mathrm{HMBC} \text { NMR data of } \\
\text { compound } \mathbf{6 b} \text { in } \mathrm{CDCl}_{3} \text {. }\end{array}$ & S25 \\
\hline 22 & Figure S11. HRMS of compound $\mathbf{6 b}$ & S26 \\
\hline 23 & Figure S12. NMR spectra of compound 2 & S27-S29 \\
\hline 24 & Figure S13. NMR spectra of compound 3 & S30-S32 \\
\hline 25 & Figure S14. NMR spectra of compound 4 & S33-S35 \\
\hline 26 & Figure S15. NMR spectra of compound $\mathbf{5 a}$ & S36-S38 \\
\hline 27 & Figure S16. NMR spectra of compound $\mathbf{5 b}$ & S39-S41 \\
\hline 28 & Figure S17. NMR spectra of compound $\mathbf{5 c}$ & S42-S44 \\
\hline 29 & Figure S18. NMR spectra of compound $\mathbf{6 a}$ & S45-S47 \\
\hline 30 & Figure S19. NMR spectra of compound $\mathbf{6 b}$ & S48-S50 \\
\hline
\end{tabular}




\section{General experimental conditions:}

Photochemical reactions were conducted on a photo reactor containing 16 lamps alternatively with PHILIPS (15W) UV-A and UV-C. Image of the photoreactor was provided in the Supporting Information of our previous publication [Org. Lett. 2020, 22, 6409-6413]. The ${ }^{1} \mathrm{H}(500 \mathrm{MHz})$ and ${ }^{13} \mathrm{C}(125 \mathrm{MHz}) \mathrm{NMR}$ spectra were recorded from Bruker ASCEND ${ }^{\mathrm{Tm}}-500 \mathrm{MHz}$, using $\mathrm{CDCl}_{3}$ and $\mathrm{CD}_{3} \mathrm{COCD}_{3}$ as solvents and the chemical shifts are expressed in $\delta(\mathrm{ppm})$, coupling constants in $\mathrm{Hz}$ with multiplicity $(\mathrm{s}=$ singlet, $\mathrm{d}=$ doublet, $\mathrm{t}=$ triplet, $\mathrm{bs}=$ broad singlet, $\mathrm{m}=$ multiplet, $\mathrm{brd}=$ broad doublet, dd = doublet of doublet). The HRMS (ESI) data were obtained from Thermo Scientific Exactive mass spectrometer. Column chromatography was performed by 100-200 and 230-400 mesh size silica gel (Merck, Darmstadt, Germany). For thin-layer chromatography (TLC) Merck precoated silica gel F254 plates were used. Spots were detected on TLC under UV light and by charring the TLC plate after spraying with $p$-anisaldehyde - sulphuric acid solution. Reagents were purchased at the highest commercial quality and used without further purification.

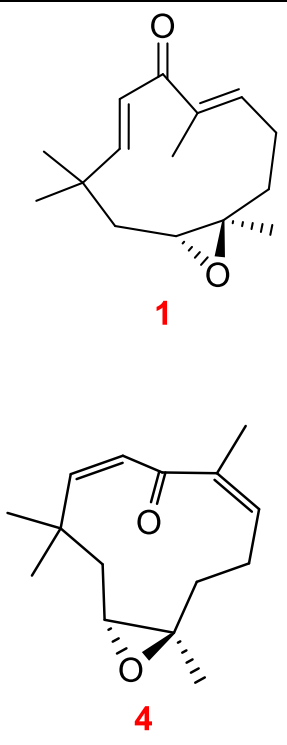

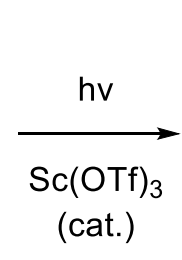

(cat.)

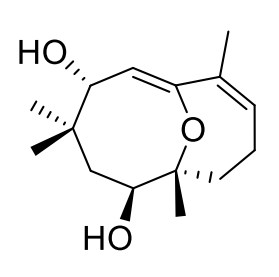

$5 a$<smiles>CC(C)C1=CC(=O)[C@@]2(C)[C@@H](O)CC[C@@H](O)[C@H]2C1</smiles>

2

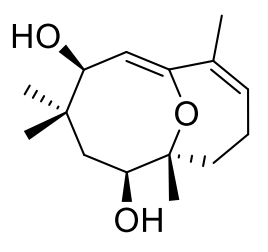

$5 b$

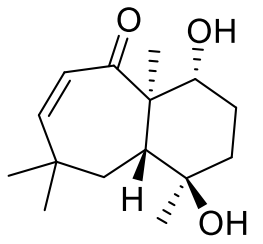

3

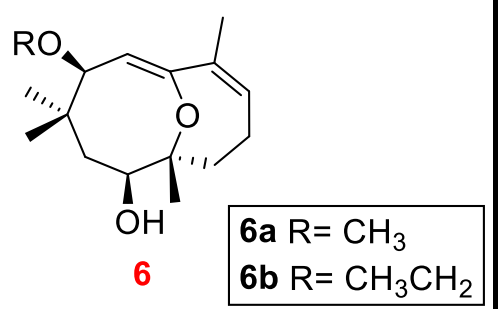

To a solution of zerumbone epoxide 1 (20 mg, solvent $10 \mathrm{~mL})$ in a quartz tube was added $\mathrm{Sc}(\mathrm{OTf})_{3}$ (0.1 equiv) and degassed with a stream of nitrogen prior to irradiation of the reaction mixture by UV-A (315-400 nm, PHILIPS (15W) lamp) in the photoreactor. After 2 hrs of irradiation with UVA, complete consumption of the starting material was observed over TLC. The reaction mixture was concentrated, and the residue was subjected to purification over silica gel column chromatography. 


\section{Compound 2:}

Compound 2 was obtained as a colorless liquid (30\% EtOAc/Hexane). ${ }^{1} \mathrm{H}$ NMR $\left(\mathrm{CD}_{3} \mathrm{COCD}_{3}, 500\right.$ MHz): $\delta 5.54(\mathrm{~s}, 1 \mathrm{H}), 3.92(\mathrm{~s}, 1 \mathrm{H}), 3.62(\mathrm{dd}, 1 \mathrm{H}, J=4.3,10.7 \mathrm{~Hz}), 3.38(\mathrm{~s}, 1 \mathrm{H}), 2.54(\mathrm{dd}, 1 \mathrm{H}, J=$ 3.5, 19.0 Hz), $2.37(\mathrm{~m}, 1 \mathrm{H}), 2.30(\mathrm{~m}, 1 \mathrm{H}), 1.73(\mathrm{dd}, 1 \mathrm{H}, J=3.8,11.7 \mathrm{~Hz}), 1.61(\mathrm{~m}, 1 \mathrm{H}), 1.41(\mathrm{~m}, 1 \mathrm{H})$, 1.51(m, 1H), $1.38(\mathrm{~m}, 1 \mathrm{H}), 1.12(\mathrm{~s}, 3 \mathrm{H}), 1.01(\mathrm{~d}, 3 \mathrm{H}, J=4.0 \mathrm{~Hz}), 0.99$ (d, 3H, $J=3.5 \mathrm{~Hz}), 0.90(\mathrm{~s}$, $3 \mathrm{H}) \mathrm{ppm} .{ }^{13} \mathrm{C}\left\{{ }^{1} \mathrm{H}\right\} \mathrm{NMR}\left(\mathrm{CD}_{3} \mathrm{COCD}_{3}, 125 \mathrm{MHz}\right): \delta 208.0,172.6,120.5,73.3,70.3,49.2,48.2,40.2$, 35.4, 26.7, 24.7, 22.9, 20.4, 19.9, 10.1 ppm. HRMS (ESI) m/z: $[\mathrm{M}+\mathrm{Na}]^{+} \mathrm{Calcd}$ for $\mathrm{C}_{15} \mathrm{H}_{24} \mathrm{NaO}_{3}$ 275.1623; Found 275.1625.

\section{Compound 3:}

Compound 3 was obtained as a colorless liquid (25\% EtOAc/Hexane). ${ }^{1} \mathrm{H} \mathrm{NMR}\left(\mathrm{CDCl}_{3}, 500 \mathrm{MHz}\right)$ : $\delta 5.99(\mathrm{~d}, 1 \mathrm{H}, J=12.7 \mathrm{~Hz}), 5.67(\mathrm{~d}, 1 \mathrm{H}, J=12.7 \mathrm{~Hz}), 3.52(\mathrm{dd}, 1 \mathrm{H}, J=4.0,11.0 \mathrm{~Hz}), 1.88(\mathrm{~d}, 1 \mathrm{H}$, $J=7.7 \mathrm{~Hz}), 1.81(\mathrm{~m}, 1 \mathrm{H}), 1.76(\mathrm{~m}, 1 \mathrm{H}), 1.73(\mathrm{~m}, 1 \mathrm{H}), 1.67(\mathrm{~m}, 1 \mathrm{H}), 1.53(\mathrm{~m}, 1 \mathrm{H}), 1.45(\mathrm{~m}, 1 \mathrm{H})$, 1.15 (s, 3H), 1.13 (s, 3H), 1.07 (s, 3H), 1.07 (s, 3H) ppm. ${ }^{13} \mathrm{C}\left\{{ }^{1} \mathrm{H}\right\}$ NMR (CDCl, $\left.125 \mathrm{MHz}\right): 211.4$, 155.3, 125.6, 72.5, 72.3, 56.0, 45.3, 39.8, 39.7, 36.2, 31.0, 27.2, 26.9, 23.7, 13.5 ppm. HRMS (ESI) $\mathrm{m} / \mathrm{z}:[\mathrm{M}+\mathrm{Na}]^{+} \mathrm{Calcd}$ for $\mathrm{C}_{15} \mathrm{H}_{24} \mathrm{NaO}_{3} 275.1623$; Found 275.1628.

\section{Compound 4:}

Compound 4 was obtained as a colorless viscous liquid (8\% EtOAc/Hexane). ${ }^{1} \mathrm{H}$ NMR ( $\mathrm{CDCl}_{3}, 500$ MHz): $\delta 6.68$ (brd, 1H, $J=9.5 \mathrm{~Hz}), 6.02(\mathrm{~d}, 1 \mathrm{H}, J=13.3 \mathrm{~Hz}), 5.77(\mathrm{~d}, 1 \mathrm{H}, J=13.3 \mathrm{~Hz}), 2.85(\mathrm{dd}$, $1 \mathrm{H}, J=11.0,2.0 \mathrm{~Hz}), 2.59(\mathrm{~m}, 1 \mathrm{H}), 2.43(\mathrm{~m}, 1 \mathrm{H}), 2.29$ (brd, $1 \mathrm{H}, J=13.2 \mathrm{~Hz}), 2.13(\mathrm{~d}, 1 \mathrm{H}, J=13.7$ $\mathrm{Hz}), 1.35(\mathrm{~m}, 1 \mathrm{H}), 1.27(\mathrm{~m}, 1 \mathrm{H}), 1.85(\mathrm{~s}, 3 \mathrm{H}), 1.19(\mathrm{~s}, 3 \mathrm{H}), 1.09(\mathrm{~s}, 3 \mathrm{H}), 0.99(\mathrm{~s}, 3 \mathrm{H}) \mathrm{ppm} .{ }^{13} \mathrm{C}\left\{{ }^{1} \mathrm{H}\right\}$ NMR ( $\left.\mathrm{CDCl}_{3}, 125 \mathrm{MHz}\right)$ : 200.7, 148.6, 143.2, 139.5, 127.5, 63.4, 60.8, 41.8, 38.7, 37.4, 33.3, 25.3, 25.3, 15.9, 11.1 ppm. HRMS (ESI) m/z: [M+Na] ${ }^{+}$Calcd for $\mathrm{C}_{15} \mathrm{H}_{22} \mathrm{NaO}_{2} 257.1517$; Found 257.1517.

\section{Compound 5a:}

Compound 5a was obtained as a colorless viscous liquid (15\% EtOAc/Hexane). ${ }^{1} \mathrm{H}$ NMR $\left(\mathrm{CDCl}_{3}\right.$, $500 \mathrm{MHz}$ ): $\delta 5.80$ (d, $1 \mathrm{H}, J=6.7 \mathrm{~Hz}), 5.57(\mathrm{~d}, 1 \mathrm{H}, J=7.5 \mathrm{~Hz}), 4.05(\mathrm{~d}, 1-\mathrm{OH}, J=10.7 \mathrm{~Hz}), 3.97$ (d, $J=9.1 \mathrm{~Hz}), 3.62(\mathrm{dd}, 1 \mathrm{H}, J=8.0,2.0 \mathrm{~Hz}), 2.49(\mathrm{~m}, 2 \mathrm{H}), 2.07(\mathrm{~m}, 2 \mathrm{H}), 1.76(\mathrm{~s}, 3 \mathrm{H}), 1.72(\mathrm{~m}, 1 \mathrm{H})$, $1.31(\mathrm{~s}, 3 \mathrm{H}), 1.11(\mathrm{~m}, 1 \mathrm{H}), 1.09(\mathrm{~s}, 3 \mathrm{H}), 0.98(\mathrm{~s}, 3 \mathrm{H}) \mathrm{ppm} .{ }^{13} \mathrm{C}\left\{{ }^{1} \mathrm{H}\right\} \mathrm{NMR}(\mathrm{CDCl} 3,125 \mathrm{MHz}): \delta$ 154.0, 133.3, 132.1, 118.8, 84.5, 76.0, 73.9, 42.2, 41.7, 38.7, 30.8, 23.9, 23.5, 21.1, 18.5 ppm. HRMS (ESI) $\mathrm{m} / \mathrm{z}:[\mathrm{M}+\mathrm{Na}]^{+}$Calcd for $\mathrm{C}_{15} \mathrm{H}_{24} \mathrm{NaO}_{3}$ 275.1623; Found 275.1618 . 


\section{Compound 5b:}

Compound $\mathbf{5 b}$ was obtained as a colorless viscous liquid (20\% EtOAc/Hexane). ${ }^{1} \mathrm{H} \mathrm{NMR}\left(\mathrm{CDCl}_{3}\right.$, $500 \mathrm{MHz}): \delta 5.79$ (brd, $1 \mathrm{H}, J=6.6 \mathrm{~Hz}), 5.11(\mathrm{~d}, 1 \mathrm{H}, J=5.4 \mathrm{~Hz}), 4.30(\mathrm{~d}, 1 \mathrm{H}, J=5.4 \mathrm{~Hz}), 3.90(\mathrm{~d}$, $1 \mathrm{H}, J=8.7 \mathrm{~Hz}), 2.50(\mathrm{t}, 1 \mathrm{H}, J=15.0 \mathrm{~Hz}), 2.20(\mathrm{~m}, 1 \mathrm{H}), 2.05(\mathrm{~m}, 1 \mathrm{H}), 2.02(\mathrm{~m}, 1 \mathrm{H}), 1.78(\mathrm{~s}, 3 \mathrm{H})$, $1.71(\mathrm{~m}, 1 \mathrm{H}), 1.31(\mathrm{dd}, 1 \mathrm{H}, J=19.0,4.1 \mathrm{~Hz}), 1.25(\mathrm{~s}, 3 \mathrm{H}), 1.08(\mathrm{~s}, 3 \mathrm{H}), 0.98(\mathrm{~s}, 3 \mathrm{H}) \mathrm{ppm} .{ }^{13} \mathrm{C}\{1 \mathrm{H}\}$ $\mathrm{NMR}\left(\mathrm{CDCl}_{3}, 125 \mathrm{MHz}\right): \delta 150.8,132.9,132.2,121.4,82.4,75.7,73.5,47.2,42.2,38.3,29.1,24.0$, 21.0, 18.2, 18.1 ppm. HRMS (ESI) m/z: [M+Na] $]^{+}$Calcd for $\mathrm{C}_{15} \mathrm{H}_{24} \mathrm{NaO}_{3}$ 275.1623; Found 275.1618.

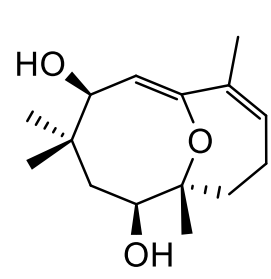

$5 b$
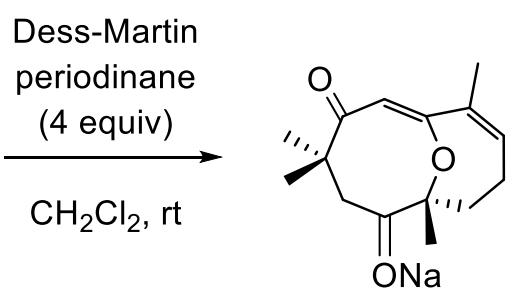

5c

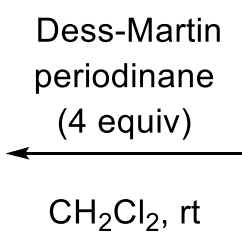

periodinane

$\mathrm{CH}_{2} \mathrm{Cl}_{2}, \mathrm{rt}$

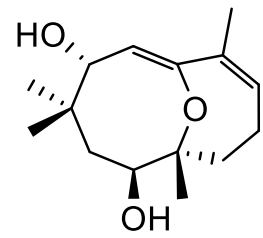

$5 a$

\section{Compound 5c:}

To a solution of compound 5a $(17 \mathrm{mg}, 0.067 \mathrm{mmol})$ in $\mathrm{CH}_{2} \mathrm{Cl}_{2}(5 \mathrm{~mL})$ was added Dess-Martin periodinane $(0.264 \mathrm{mmol}, 4$ equiv) and the reaction mixture was stirred at room temperature. After $6 \mathrm{hrs}$, the reaction mixture was diluted with $\mathrm{CH}_{2} \mathrm{Cl}_{2}(15 \mathrm{~mL})$ and added saturated aqueous $\mathrm{Na}_{2} \mathrm{~S}_{2} \mathrm{O}_{3}$ solution $(10 \mathrm{~mL})$. The organic layer was again washed with saturated aqueous $\mathrm{NaHCO}_{3}$ solution $(10$ $\mathrm{mL}$ ) and the organic layer was dried over anhydrous $\mathrm{Na}_{2} \mathrm{SO}_{4}$ and concentrated. The residue was subjected to purification over silica gel column chromatography (5\% EtOAc/hexane) to afford compound 5c (13 mg, 78\%) as a pale yellow viscous liquid. Same reaction was conducted with compound $\mathbf{5 b}(20 \mathrm{mg}, 0.79 \mathrm{mmol})$, which also afforded compound 5c $(16 \mathrm{mg}, 81 \%) .{ }^{1} \mathrm{H} \mathrm{NMR}$ $\left(\mathrm{CDCl}_{3}, 500 \mathrm{MHz}\right): \delta 5.82(\mathrm{~d}, 1 \mathrm{H}, J=8.0 \mathrm{~Hz}), 5.56(\mathrm{~s}, 1 \mathrm{H}), 2.91(\mathrm{~d}, 1 \mathrm{H}, J=12.3 \mathrm{~Hz}), 2.69(\mathrm{~d}, 1 \mathrm{H}$, $J=12.4 \mathrm{~Hz}), 2.44(\mathrm{~m}, 1 \mathrm{H}), 2.18(\mathrm{~m}, 1 \mathrm{H}), 2.07$ (m, 1H), $1.78(\mathrm{~s}, 3 \mathrm{H}), 1.74(\mathrm{~m}, 1 \mathrm{H}), 1.35$ (s, 3H), $1.20(\mathrm{~s}, 3 \mathrm{H}), 1.12(\mathrm{~s}, 3 \mathrm{H}) \mathrm{ppm} .{ }^{13} \mathrm{C}\left\{{ }^{1} \mathrm{H}\right\} \mathrm{NMR}\left(\mathrm{CDCl}_{3}, 125 \mathrm{MHz}\right): \delta 212.8,208.4,157.0,134.7$, 132.0, 116.6, 87.8, 47.8, 47.8, 33.8, 27.8, 25.4, 24.0, 22.5, 22.2 ppm. HRMS (ESI) m/z: [M+Na] ${ }^{+}$ Calcd for $\mathrm{C}_{15} \mathrm{H}_{20} \mathrm{NaO}_{3}$ 271.1310; Found 271.1304. 


\section{Compound 6a:}

Compound 6a was obtained as a colorless viscous liquid (5\% EtOAc/Hexane). ${ }^{1} \mathrm{H}$ NMR $\left(\mathrm{CDCl}_{3}\right.$, $500 \mathrm{MHz}$ ): $\delta 5.79$ (brd, $1 \mathrm{H}, J=6.6 \mathrm{~Hz}), 5.12(\mathrm{~d}, 1 \mathrm{H}, J=5.5 \mathrm{~Hz}), 3.92(\mathrm{~d}, 1 \mathrm{H}, J=8.5 \mathrm{~Hz}), 3.72(\mathrm{~d}$, $1 \mathrm{H}, J=5.5 \mathrm{~Hz}), 3.27(\mathrm{~s}, 3 \mathrm{H}), 2.51(\mathrm{t}, 1 \mathrm{H}, J=14.5 \mathrm{~Hz}), 2.19(\mathrm{~m}, 1 \mathrm{H}), 2.07(\mathrm{~m}, 1 \mathrm{H}), 2.03(\mathrm{~m}, 1 \mathrm{H})$, $1.79(\mathrm{~s}, 3 \mathrm{H}), 1.72(\mathrm{~m}, 1 \mathrm{H}), 1.29(\mathrm{~m}, 1 \mathrm{H}), 1.26(\mathrm{~s}, 3 \mathrm{H}), 1.06(\mathrm{~s}, 3 \mathrm{H}), 0.96(\mathrm{~s}, 3 \mathrm{H}) \mathrm{ppm},{ }^{13} \mathrm{C}\left\{{ }^{1} \mathrm{H}\right\} \mathrm{NMR}$ $\left(\mathrm{CDCl}_{3}, 125 \mathrm{MHz}\right): \delta 152.5,132.7,132.4,121.2,85.0,82.5,73.5,57.4,47.8,42.3,38.2,29.2,24.0$, 21.1, 19.1, 18.2 ppm. HRMS (ESI) m/z: [M+Na] ${ }^{+} \mathrm{Calcd}$ for $\mathrm{C}_{16} \mathrm{H}_{26} \mathrm{NaO}_{3} 289.1780$; Found 289.1778.

\section{Compound 6b:}

Compound $\mathbf{6 b}$ was obtained as a colorless viscous liquid (3\% EtOAc/Hexane). ${ }^{1} \mathrm{H}$ NMR $\left(\mathrm{CDCl}_{3}\right.$, $500 \mathrm{MHz}): 5.70(\mathrm{~d}, 1 \mathrm{H}, J=7.4 \mathrm{~Hz}), 5.07(\mathrm{~d}, 1 \mathrm{H}, J=5.3 \mathrm{~Hz}), 3.85(\mathrm{~d}, 1 \mathrm{H}, J=8.6 \mathrm{~Hz}), 3.74(\mathrm{~d}, J=$ $5.0 \mathrm{~Hz}), 3.49(\mathrm{~m}, 1 \mathrm{H}), 3.22(\mathrm{~m}, 1 \mathrm{H}), 2.43(\mathrm{~m}, 1 \mathrm{H}), 2.12(\mathrm{dd}, 1 \mathrm{H}, J=14.9,8.8 \mathrm{~Hz}), 1.99(\mathrm{~m}, 1 \mathrm{H})$, $1.97(\mathrm{~m}, 1 \mathrm{H}), 1.71(\mathrm{~s}, 3 \mathrm{H}), 1.64(\mathrm{~m}, 1 \mathrm{H}), 1.22(\mathrm{~m}, 1 \mathrm{H}), 1.18(\mathrm{~s}, 3 \mathrm{H}), 1.07$ (t, 1H, J = 7.6 Hz), 0.99 (s, 3H), 0.89 (s, 3H) ppm. ${ }^{13} \mathrm{C}\left\{{ }^{1} \mathrm{H}\right\}$ NMR $\left(\mathrm{CDCl}_{3}, 125 \mathrm{MHz}\right): \delta$ 151.8, 132.4, 128.9, 122.0, 82.9, 82.4, 73.5, 64.9, 47.8, 42.3, 38.2, 29.3, 24.0, 21.1, 19.2, 18.2, 15.3 ppm. HRMS (ESI) m/z: $[\mathrm{M}+\mathrm{Na}]^{+}$ Calcd for $\mathrm{C}_{17} \mathrm{H}_{28} \mathrm{NaO}_{3}$ 303.1936; Found 303.1940.

Scheme S1. Geometrical isomers of zerumbone epoxide $\mathbf{1}$ that afford compound $\mathbf{3}$ with cis-ring junction.

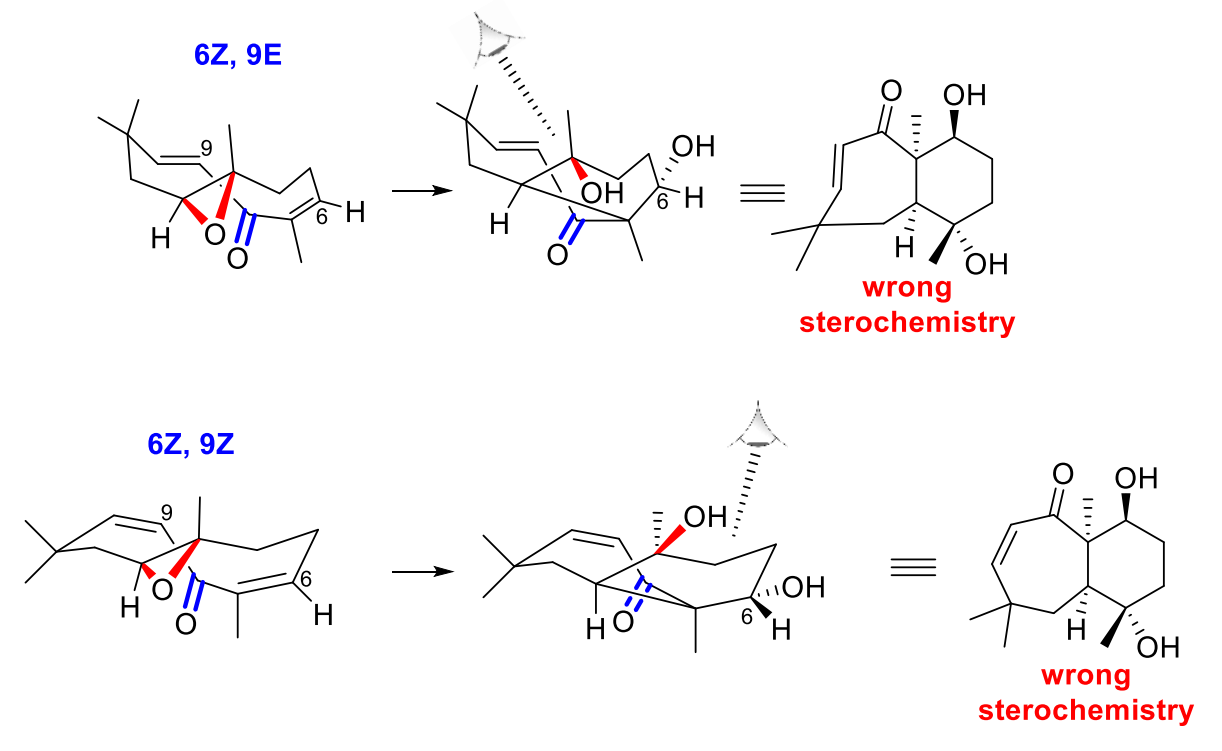


Scheme S2. Photoirradiation attempt made on 10a, a functionalized C13(OAc) of zerumbone epoxide 1<smiles>CC1=CCC(C)(C)/C=C/C(=O)C(C)=CCC1</smiles>

7

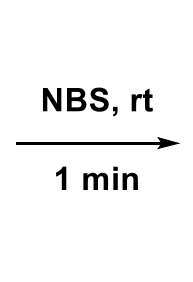<smiles>C=C1CC/C=C(\C)C(=O)/C=C/C(C)(C)CC1Br</smiles>

8
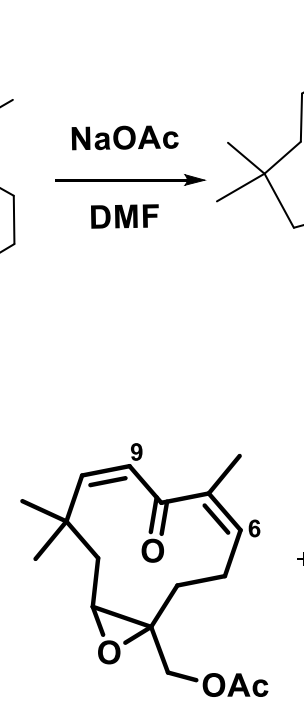

$6 Z, 9 Z$

$10 \mathrm{~b}$<smiles>CC(=O)OCC(=CCC(C)(C)C=CC(=O)C(C)=CCC(=O)OC(C)(C)C)CCC(C)(C)C</smiles>

9<smiles>CC(=O)OCC12CCC=C(C)C(=O)C=CC(C)(C)CC1O2</smiles>

$10 \mathrm{a}$

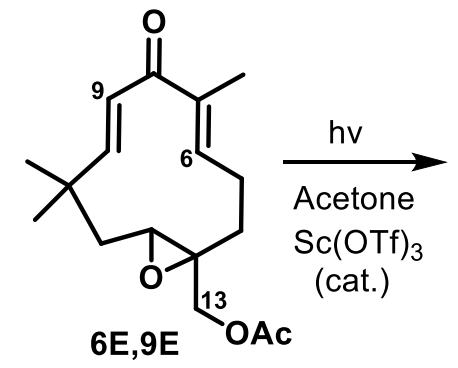

$10 \mathrm{a}$<smiles></smiles>

$6 \mathrm{E}, 9 \mathrm{Z}$

${ }^{1} \mathrm{H}(500 \mathrm{MHz})$ NMR data of compound 10a and inseparable mixture of $10 \mathbf{b} \& \mathbf{1 0} \mathbf{c}$ in $\mathrm{CDCl}_{3}$

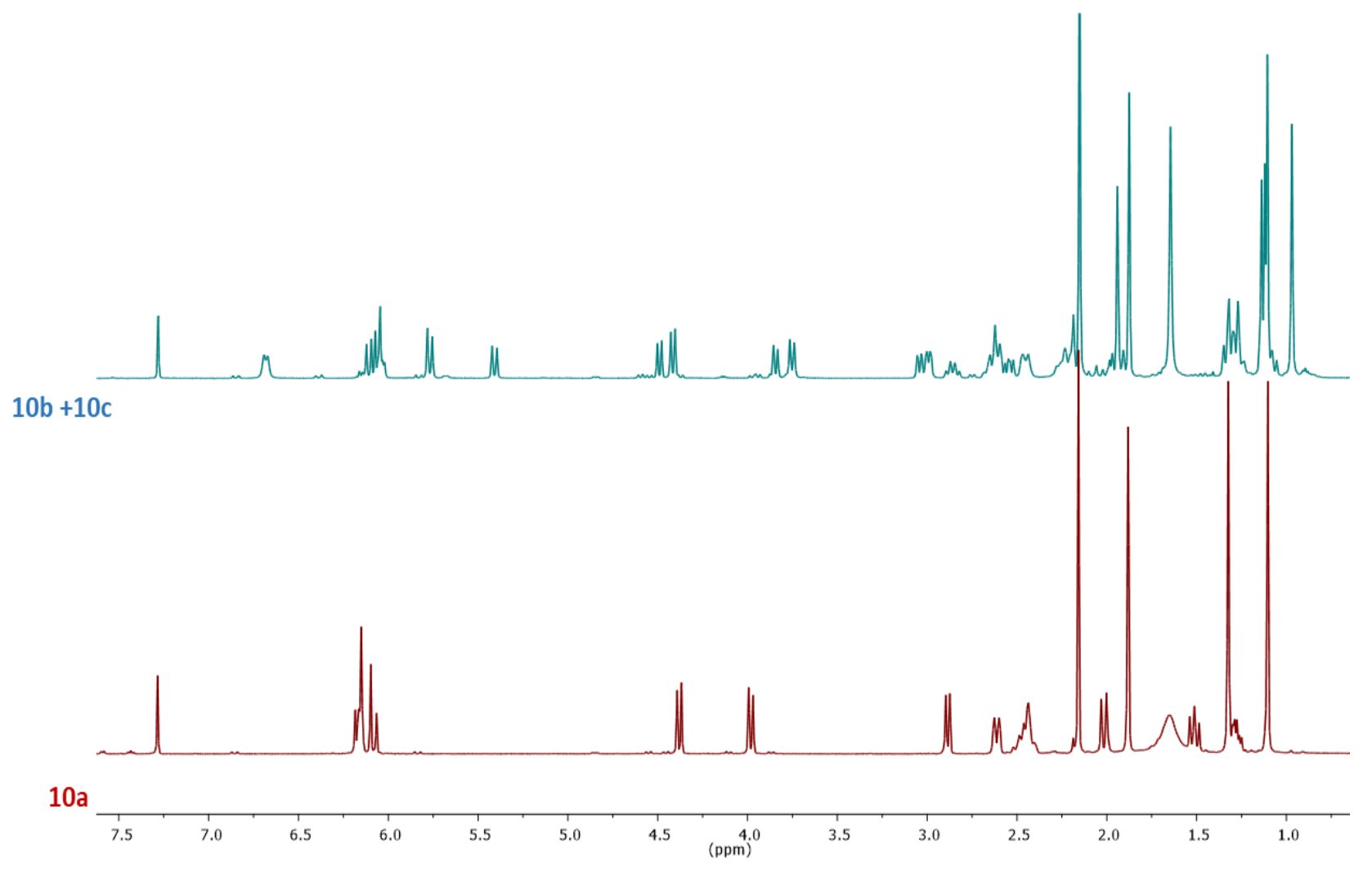


Table S1. ${ }^{1} \mathrm{H}(500 \mathrm{MHz}),{ }^{13} \mathrm{C}(125 \mathrm{MHz}), \mathrm{COSY}$ and HMBC NMR data of compound $\mathbf{2}$ in acetoned6.

\begin{tabular}{|c|c|c|c|c|c|}
\hline Position & $\delta_{\mathbf{H}}(J)$ & $\delta_{\mathrm{C}}$ & COSY & НMBC & \\
\hline 1 & $\begin{array}{c}2.54 \mathrm{dd}(3.5,19.0) \\
2.30 \mathrm{~m}\end{array}$ & 24.7 & & $7,9,10,11$ & \\
\hline 2 & $1.73 \mathrm{dd}(3.8,11.7)$ & 49.2 & 1 & $12,13,1,7,3$ & \\
\hline 3 & & 70.3 & & & \\
\hline 4 & $\begin{array}{l}1.61 \mathrm{~m} \\
1.41 \mathrm{~m}\end{array}$ & 40.2 & 5 & $2,6,3$ & 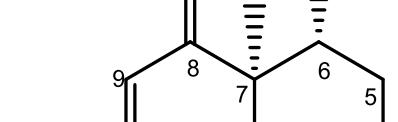 \\
\hline 5 & $\begin{array}{l}1.51 \mathrm{~m} \\
1.38 \mathrm{~m}\end{array}$ & 26.7 & & $7,6,3$ & $\overbrace{11}^{14}$ \\
\hline 6 & $3.62 \mathrm{dd}(4.3,10.7)$ & 73.3 & 5 & & 15 \\
\hline 7 & & 48.2 & & & \\
\hline 8 & & 208.0 & & & \\
\hline 9 & $5.54 \mathrm{~s}$ & 120.5 & & $1,11,7$ & \\
\hline 10 & & 172.6 & & & \\
\hline 11 & $2.37 \mathrm{~m}$ & 35.4 & 14,15 & $14,1,9,10$ & \\
\hline 12 & $0.90 \mathrm{~s}$ & 10.1 & & $2,6,8$ & \\
\hline 13 & $1.12 \mathrm{~s}$ & 22.9 & & $3,4,2$ & \\
\hline 14 & $1.01 \mathrm{~d}(4.0)$ & 20.4 & & $11,15,10$ & \\
\hline 15 & $0.99 \mathrm{~d}(3.5)$ & 19.9 & & $11,14,10$ & \\
\hline OH-C6 & $3.92 \mathrm{~s}$ & & & 5,6 & \\
\hline OH-C3 & $3.38 \mathrm{~s}$ & & & 13 & \\
\hline
\end{tabular}

\section{Structure elucidation of compound 2:}

The major product 2 exhibited only one olefinic proton $\mathrm{H}-9\left(\delta_{\mathrm{H}} 5.54 \mathrm{ppm}\right)$, two exchangeable protons $\left(\delta_{\mathrm{H}} 3.92\right.$ and $\left.3.38 \mathrm{ppm}\right)$, and a ketone carbonyl C8 $\left(\delta_{\mathrm{C}} 208.0 \mathrm{ppm}\right)$. HR-ESI-MS measured at $\mathrm{m} / \mathrm{z}$ 275.1625, which along with ${ }^{1} \mathrm{H}$ and ${ }^{13} \mathrm{C}$ NMR data indicates $[\mathrm{M}+\mathrm{Na}]^{+}$ion, suggesting compound 2 as a transannular cyclized product with molecular formula $\mathrm{C}_{15} \mathrm{H}_{24} \mathrm{O}_{3}$. The presence of two exchangeable protons indicates the incorporation of a hydroxyl group in the molecule at methine C6 $\left(\delta_{\mathrm{C}} 73.3 \mathrm{ppm}\right)$ from water due to the usage of undistilled acetone in the reaction. ${ }^{3} J$ COSY correlations were observed for H-6 $\left(\delta_{\mathrm{H}} 3.62 \mathrm{ppm}\right)$ with H-5 $\left(\mathrm{CH}_{2}\right)$; and H-5 with $\mathrm{H}-4\left(\mathrm{CH}_{2}\right)$. The presence of $\mathrm{HMBC}$ correlations for exchangeable proton $\left(\delta_{\mathrm{H}} 3.92 \mathrm{ppm}\right)$ of a hydroxyl group with 
C5, C6 confirms its presence on methine $\mathrm{C} 6$, leading to the first partial structure $\mathrm{C} 6(\mathrm{OH})-\mathrm{C} 5-\mathrm{C} 4$. The presence of $\mathrm{HMBC}$ correlations for $\mathrm{H}-5$ with $\mathrm{C} 3, \mathrm{C} 6$, and $\mathrm{C} 7$; $\mathrm{H}-4$ with $\mathrm{C} 3, \mathrm{C} 6$, and $\mathrm{C} 2$; methyl $\mathrm{H}-13$ with $\mathrm{C} 2, \mathrm{C} 3$, and $\mathrm{C} 4$ indicates the second partial structure $\mathrm{C} 7-\mathrm{C} 6-\mathrm{C} 5-\mathrm{C} 4-\mathrm{C} 3(\mathrm{C} 13)-\mathrm{C} 2$. A chemical shift at $\delta_{\mathrm{C}} 70.3 \mathrm{ppm}$ indicates the presence of the second hydroxyl group on quaternary carbon $\mathrm{C} 3$. The presence of $\mathrm{HMBC}$ correlations for methyl $\mathrm{H}-12$ with ketone $\mathrm{C} 8, \mathrm{C} 2$, and $\mathrm{C} 6$ indicate the presence of methyl $\mathrm{C} 12, \mathrm{C} 8$ on quaternary carbon $\mathrm{C} 7$; and $\mathrm{HMBC}$ correlations for $\mathrm{H}-2$ with $\mathrm{C} 7$, $\mathrm{C} 12$, and $\mathrm{C} 13$ confirms $\mathrm{C} 7-\mathrm{C} 2$ bond, thus, confirming the key cyclohexyl fragment of the compound. The presence of ${ }^{3} \mathrm{~J}$ COSY correlations for H-11 with H-14, H-15, and HMBC correlation of H-14 and $\mathrm{H}-15$ with $\mathrm{sp}^{2}$ hybridized quaternary carbon $\mathrm{C} 10$ indicates an isopropyl group on $\mathrm{C} 10$. The presence of ${ }^{3} J$ COSY correlations for $\mathrm{H}-1$ with $\mathrm{H}-2$, and $\mathrm{HMBC}$ correlations with $\mathrm{sp}^{2}$ hybridized methine $\mathrm{C} 9, \mathrm{C} 10$, and $\mathrm{C} 11$ confirm methylene $\mathrm{H}-1$ as an allylic carbon, in agreement with its chemical shift in ${ }^{1} \mathrm{H}$ NMR. Finally, the presence of HMBC correlations for $\mathrm{H}-11$ with $\mathrm{C} 9$ and $\mathrm{C} 1$, $\mathrm{H}-9$ with $\mathrm{C} 11, \mathrm{C} 1$ and $\mathrm{C} 7$, clearly indicates the presence of a cyclohexenone ring fused with cyclohexyl ring at $\mathrm{C} 7$ and $\mathrm{C} 2$. 
Figure S1. 1D NOE spectra of compound 2

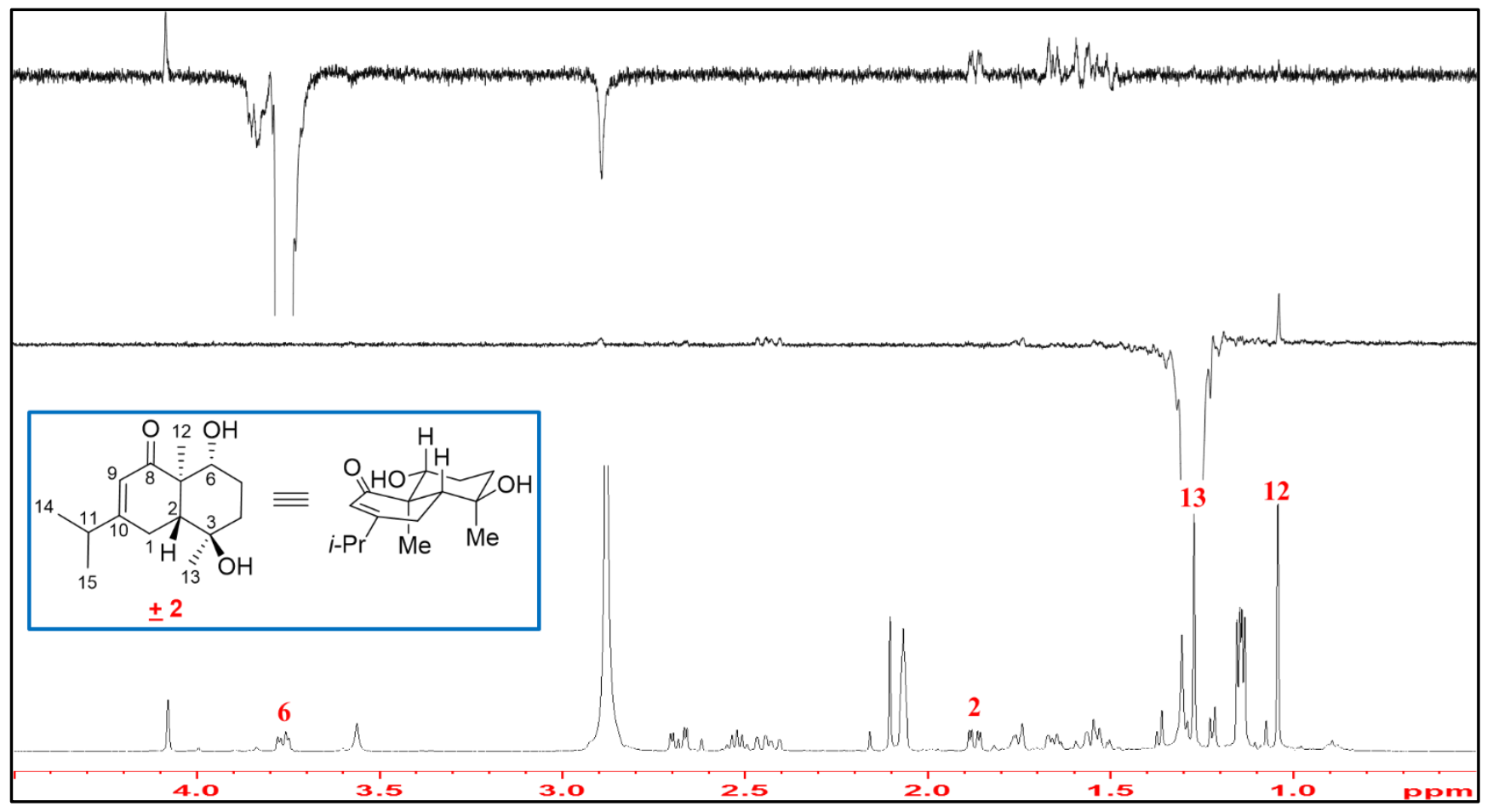

Figure S2. HRMS of compound 2

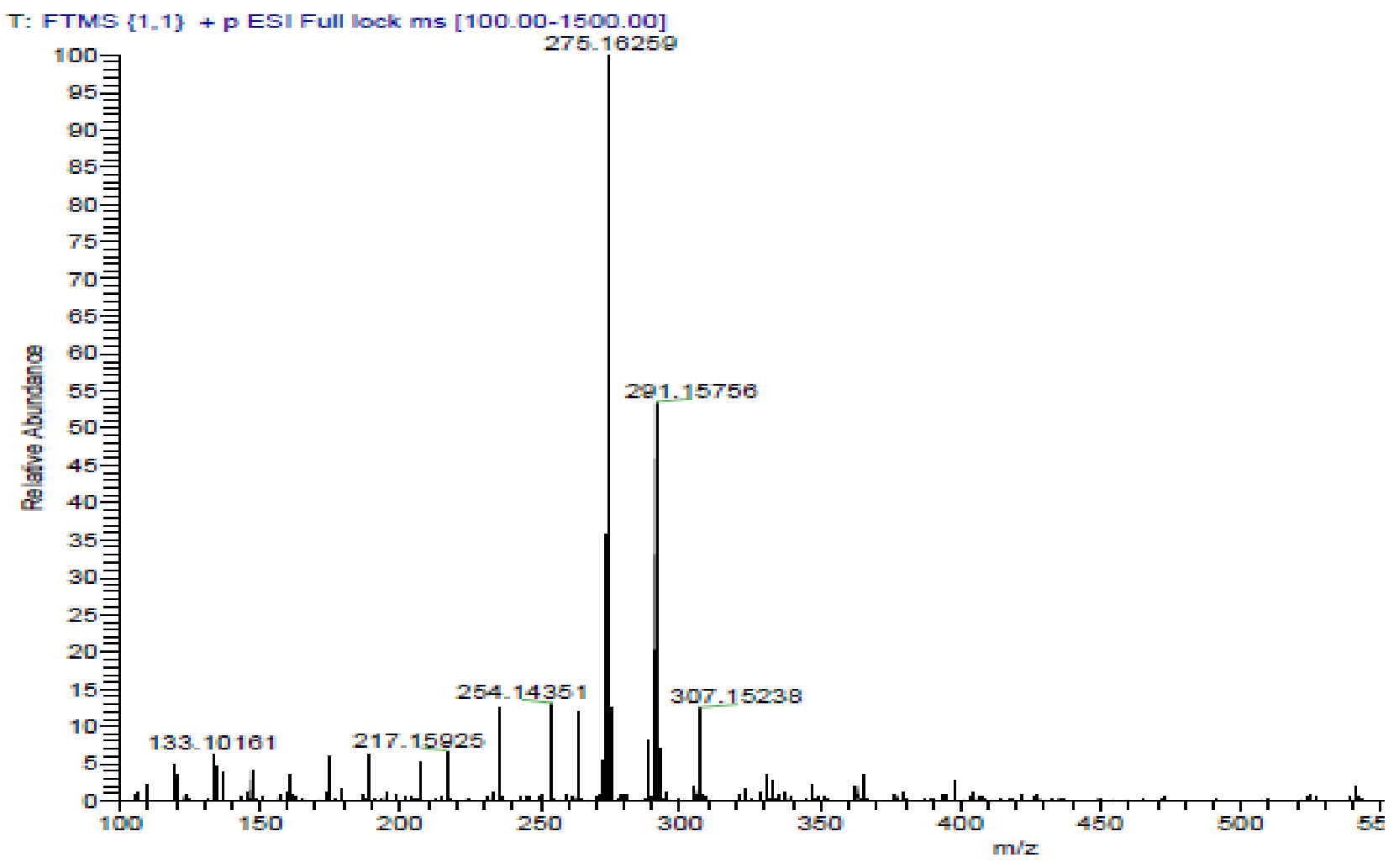


Table S2. ${ }^{1} \mathrm{H}(500 \mathrm{MHz}),{ }^{13} \mathrm{C}(125 \mathrm{MHz}), \mathrm{COSY}$ and $\mathrm{HMBC}$ NMR data of compound $\mathbf{3}$ in $\mathrm{CDCl}_{3}$

\begin{tabular}{|c|c|c|c|c|c|}
\hline Position & $\delta_{\mathbf{H}}(J)$ & $\delta \mathrm{c}$ & COSY & НMBC & \\
\hline 1 & $\begin{array}{c}1.76 \mathrm{~m}, 1.67 \\
\mathrm{~m}\end{array}$ & 36.2 & & $\begin{array}{c}15,14,11,2,7, \\
3,10\end{array}$ & \\
\hline 2 & $1.88 \mathrm{~d}(7.7)$ & 45.3 & 1 & $\begin{array}{c}7,12,13,1,6 \\
8,4\end{array}$ & \\
\hline 3 & & 72.3 & & & \\
\hline 4 & $\begin{array}{l}1.45 \mathrm{~m} \\
1.73 \mathrm{~m}\end{array}$ & 39.7 & & $5,13,3$ & \\
\hline 5 & $\begin{array}{l}1.53 \mathrm{~m} \\
1.81 \mathrm{~m}\end{array}$ & 27.2 & 6 & $4,7,3$ & \\
\hline 6 & $\begin{array}{c}3.52 \mathrm{dd}(4.0, \\
11.0)\end{array}$ & 72.5 & & $8,12,5,7$ & \\
\hline 7 & & 56.0 & & & \\
\hline 8 & & 211.4 & & & \\
\hline 9 & $5.67 \mathrm{~d}(12.7)$ & 125.6 & 10 & $11,7,10$ & \\
\hline 10 & $5.99 \mathrm{~d}(12.7)$ & 155.3 & 1 & $8,14,15,11,1$ & \\
\hline 11 & & 39.8 & & & \\
\hline 12 & $1.15 \mathrm{~s}$ & 13.5 & & $8,2,7,6$ & \\
\hline 13 & $1.13 \mathrm{~s}$ & 23.7 & & $4,2,3$ & \\
\hline 14 & $1.07 \mathrm{~s}$ & 31.0 & & $15,1,11,10$ & \\
\hline 15 & $1.07 \mathrm{~s}$ & 26.9 & & $14,1,11,10$ & \\
\hline
\end{tabular}

\section{Structure elucidation of compound 3:}

HR-ESI-MS of the minor product 3 was measured at $\mathrm{m} / \mathrm{z} 275.1628[\mathrm{M}+\mathrm{Na}]+$ ion, which indicates compound $\mathbf{3}$ as an isomer of compound 2. 2D NMR data of compound $\mathbf{3}$ (Table S2) revealed the presence of a similar cyclohexane ring as in compound $\mathbf{2}$. The presence of two olefinic protons, $\mathrm{H}-$ $10(\delta \mathrm{H} 5.99 \mathrm{ppm})$ and $\mathrm{H}-9(\delta \mathrm{H} 5.67 \mathrm{ppm})$, with cis-configuration, revealed from coupling constant and presence of gem-dimethyl $\mathrm{C} 14, \mathrm{C} 15$ on quaternary carbon $\mathrm{C} 11$, revealed from HMBC correlations, suggest the presence of enone akin to zerumbone. Further confirmation by the presence of $\mathrm{HMBC}$ correlations for $\mathrm{H}-1$ with $\mathrm{C} 11, \mathrm{C} 15, \mathrm{C} 10$, and $\mathrm{C} 7$; $\mathrm{H}-10$ with $\mathrm{C} 14, \mathrm{C} 1$, and $\mathrm{C} 8$; and $\mathrm{H}-9$ 
with $\mathrm{C} 11$ and $\mathrm{C} 7$ revealed the bicyclic structure of compound $\mathbf{3}$ by transannular cyclization, fused at $\mathrm{C} 7$ and C2. Structurally, compound $\mathbf{2}$ can be considered as derived from compound $\mathbf{3}$ by a rearrangement reaction, hence, bearing the same stereochemistry.

Figure S3. HRMS of compound 3

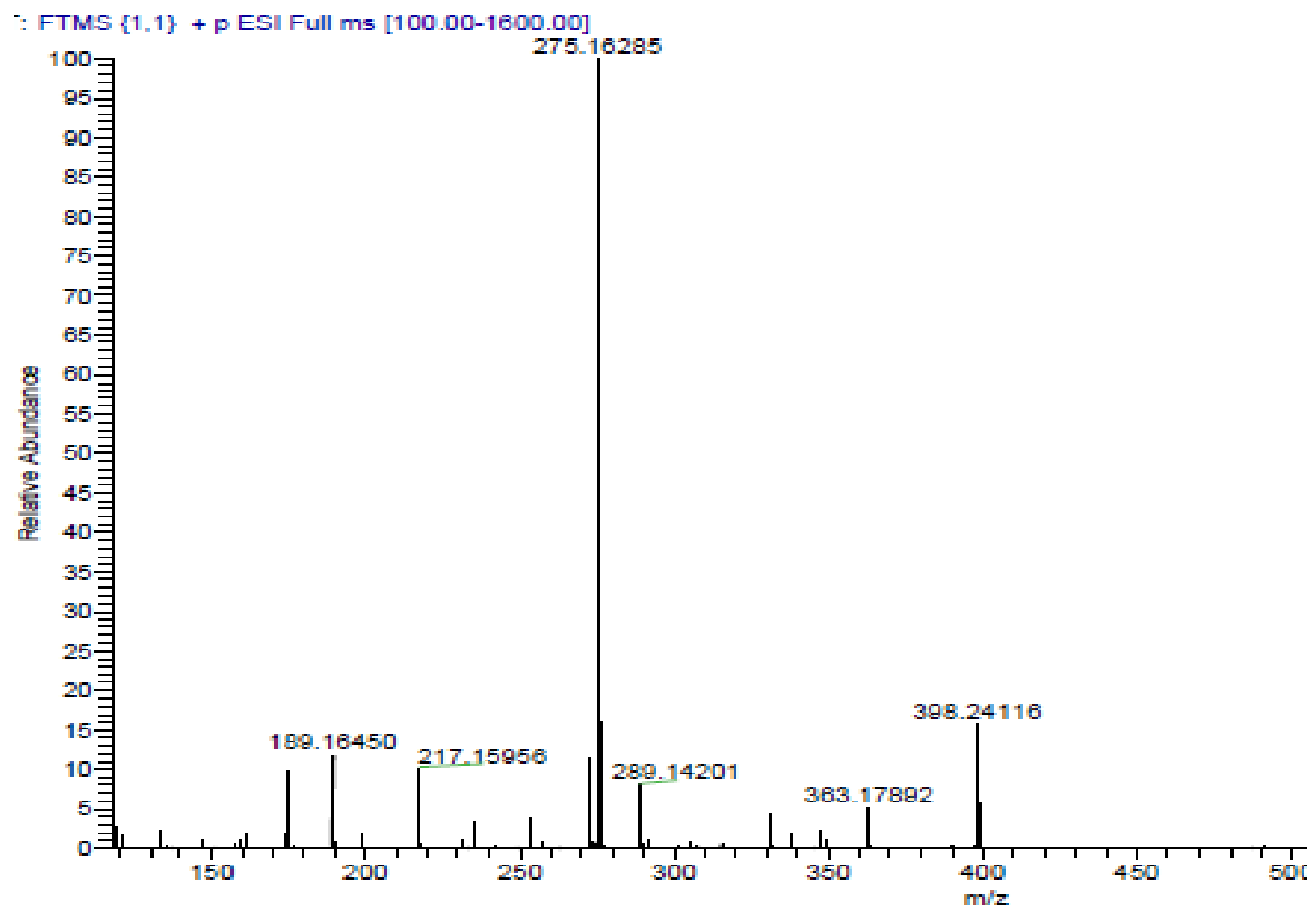


Table S3. ${ }^{1} \mathrm{H}(500 \mathrm{MHz}),{ }^{13} \mathrm{C}(125 \mathrm{MHz}), \mathrm{COSY}$ and $\mathrm{HMBC}$ NMR data of compound 4 in $\mathrm{CDCl}_{3}$

\begin{tabular}{|c|c|c|c|c|}
\hline Position & $\delta_{\mathbf{H}}(\boldsymbol{J})$ & $\delta_{\mathrm{C}}$ & COSY & HMBC \\
\hline 1 & $\begin{array}{c}1.27 \mathrm{~m}, \\
2.13 \mathrm{~d}(13.7)\end{array}$ & 41.8 & & $10,2,11$ \\
\hline 2 & $2.85 \mathrm{dd}(11,2)$ & 63.4 & 1 & 1 \\
\hline 3 & & 60.8 & & \\
\hline 4 & $\begin{array}{c}2.29 \text { brd (13.2), } \\
1.35 \mathrm{~m}\end{array}$ & 38.7 & & $13,5,2,6,3$ \\
\hline 5 & $\begin{array}{l}2.59 \mathrm{~m} \\
2.43 \mathrm{~m}\end{array}$ & 25.3 & 4 & 4 \\
\hline 6 & 6.68 brd (9.5) & 148.6 & 12,5 & \\
\hline 7 & & 139.5 & & \\
\hline 8 & & 200.7 & & \\
\hline 9 & $6.02 \mathrm{~d}(13.3)$ & 127.5 & 10 & 11,10 \\
\hline 10 & $5.77 \mathrm{~d}(13.3)$ & 143.2 & & $14,15,11,8$ \\
\hline 11 & & 37.4 & & \\
\hline 12 & $1.85 \mathrm{~s}$ & 11.1 & & $7,6,8$ \\
\hline 13 & $1.19 \mathrm{~s}$ & 15.9 & & $4,3,2$ \\
\hline 14 & $0.99 \mathrm{~s}$ & 25.3 & & $10,1,11,15$ \\
\hline 15 & $1.09 \mathrm{~s}$ & 33.3 & & $10,1,11,14$ \\
\hline
\end{tabular}


Figure S4. HRMS of compound 4

Г: FTMS $\{1,1\}+p$ ESI Full ms [100.00-1800.00]

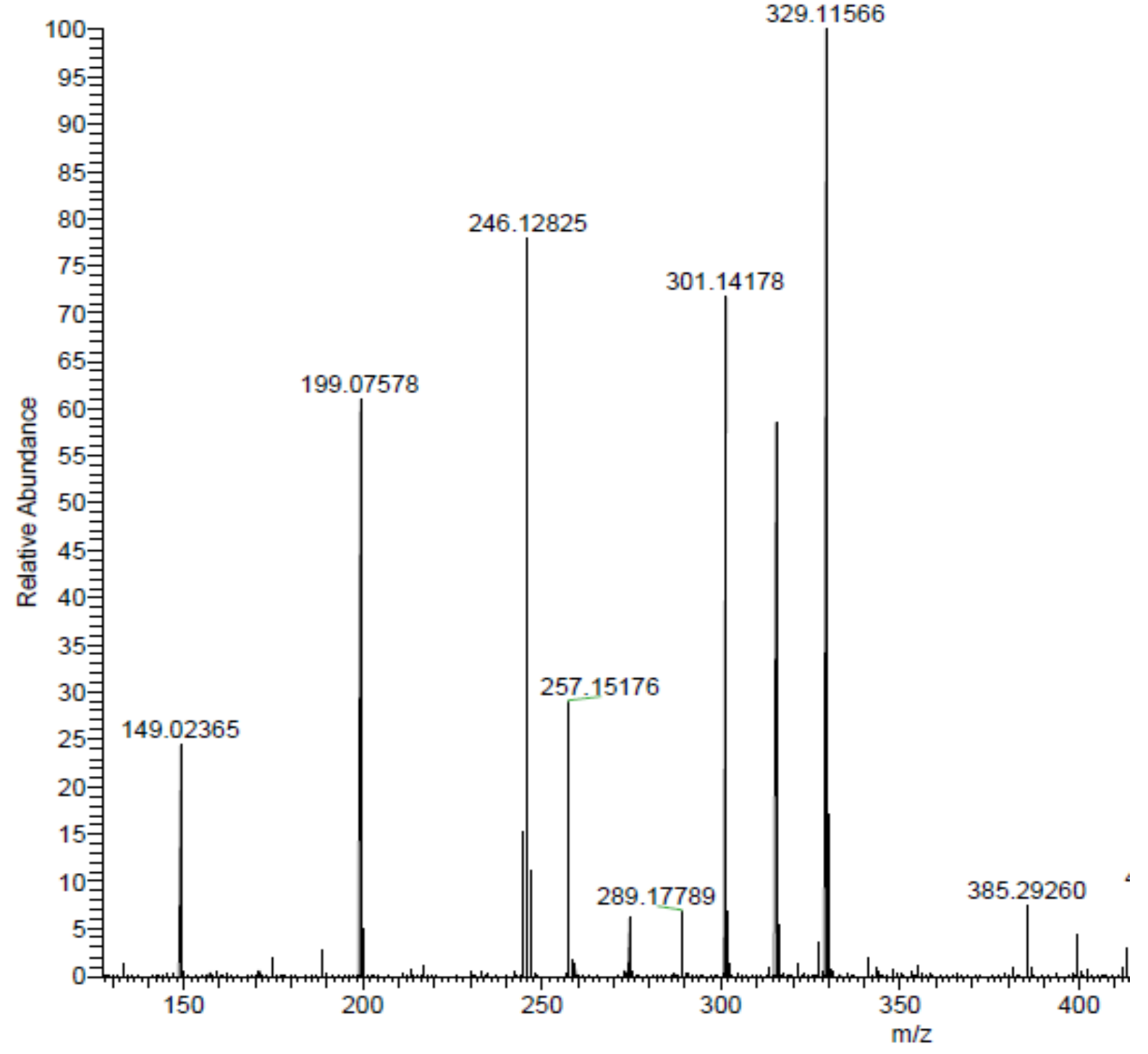


Figure S5. ${ }^{1} \mathrm{H}$ NMR spectra of different geometrical isomers of zerumbone-2,3-epoxide.
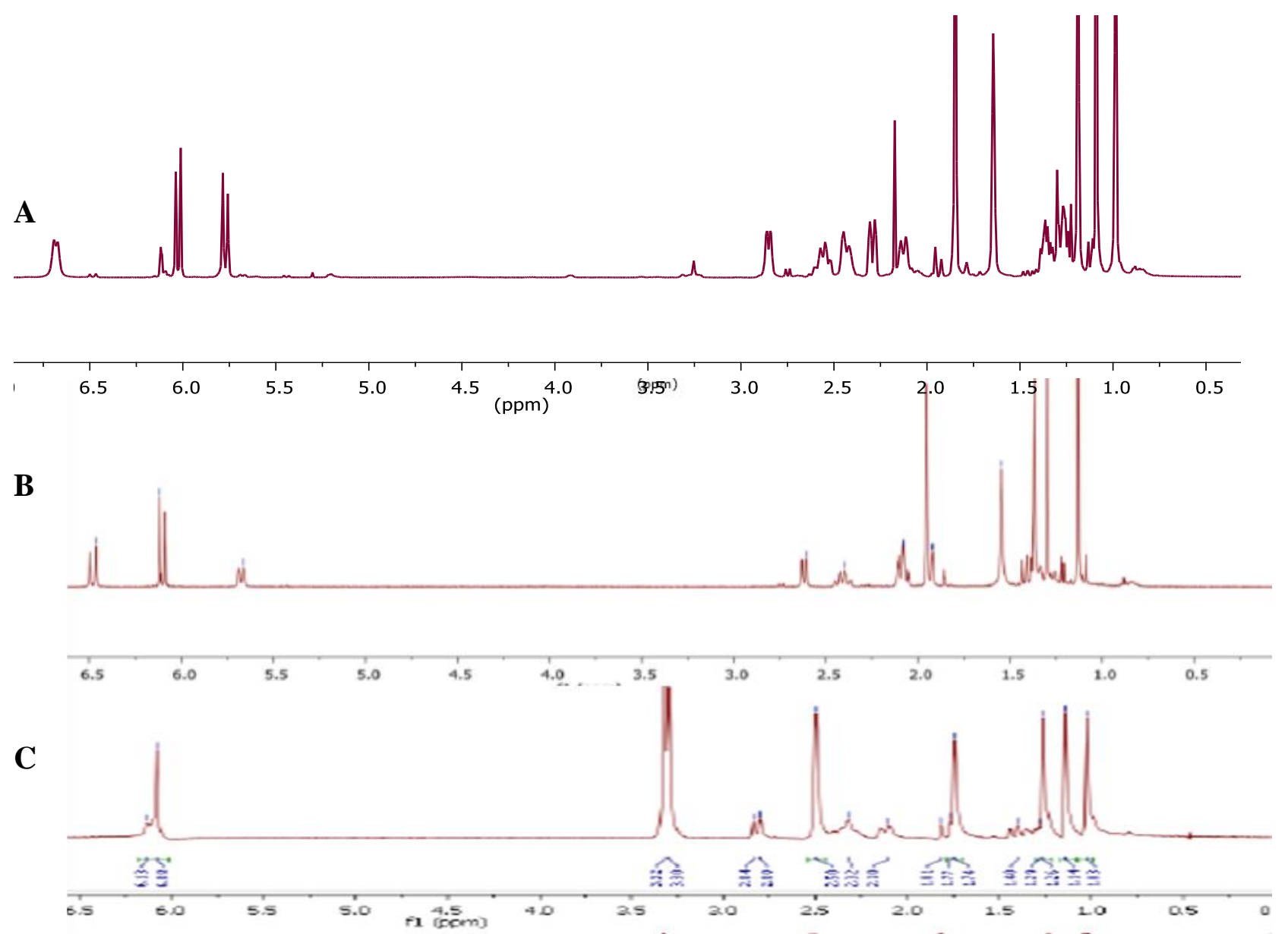

D

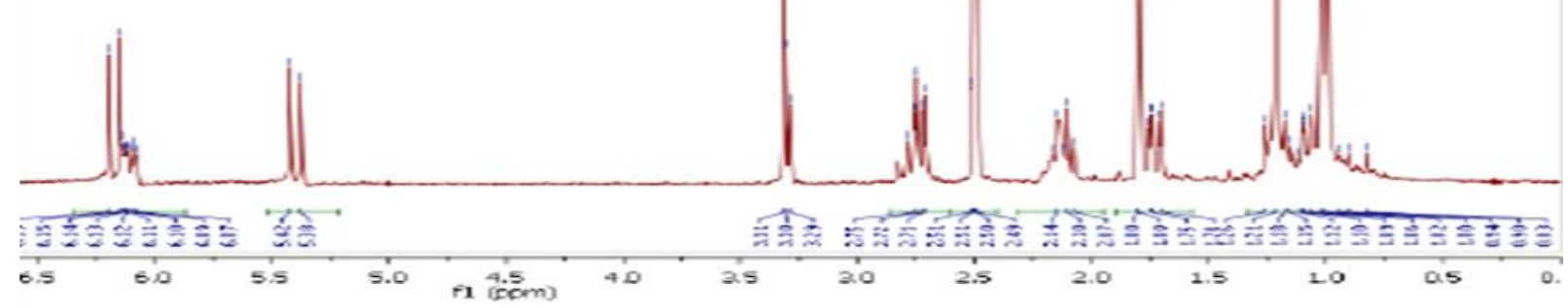

$\mathbf{A}=$ Present work, $(6 Z, 9 Z)$-zerumbone-2,3-epoxide

$\mathbf{B}=$ Craig et al., $J$. Nat. Prod. 2020; 83, 1473-1479, (6Z,9E)-zerumbone-2,3-epoxide

$\mathbf{C}=$ Appendino et al., Eur. J. Org. Chem. 2015; 3721-3726, (6E,9E)-zerumbone-2,3-epoxide

D = Appendino et al., Eur. J. Org. Chem. 2015; 3721-3726, (6E,9Z)-zerumbone-2,3-epoxide 
Table S4. ${ }^{1} \mathrm{H}(500 \mathrm{MHz}),{ }^{13} \mathrm{C}(125 \mathrm{MHz}), \mathrm{COSY}$ and $\mathrm{HMBC}$ NMR data of compound $\mathbf{5 a}$ in $\mathrm{CDCl}_{3}$

\begin{tabular}{|c|c|c|c|c|c|}
\hline Position & $\delta_{\mathbf{H}}(J)$ & $\delta_{\mathrm{C}}$ & COSY & HMBC & \\
\hline 1 & $\begin{array}{l}2.49 \mathrm{~m}, \\
1.11 \mathrm{~m}\end{array}$ & 41.7 & & $3,11,2,15$ & \\
\hline 2 & $3.97 \mathrm{~d}(9.1)$ & 73.9 & 1 & 13 & \\
\hline 3 & & 84.5 & & & \\
\hline 4 & $\begin{array}{l}2.07 \mathrm{~m} \\
1.72 \mathrm{~m}\end{array}$ & 42.2 & & $13,5,2$ & \\
\hline 5 & $\begin{array}{l}2.49 \mathrm{~m} \\
2.07 \mathrm{~m}\end{array}$ & 23.9 & 4 & & ${ }_{\mathrm{OH}}{ }_{13}$ \\
\hline 6 & $5.80 \mathrm{~d}(6.7)$ & 133.3 & 12,5 & & \\
\hline 7 & & 132.1 & & & \\
\hline 8 & & 154.0 & & & \\
\hline 9 & $5.57 \mathrm{~d}(7.5)$ & 118.8 & 10 & $10,7,8,11$ & \\
\hline 10 & $3.62 \mathrm{dd}(8.0,2.0)$ & 76.0 & 1 & $9,1,8$ & \\
\hline 11 & & 38.7 & & & \\
\hline 12 & $1.76 \mathrm{~s}$ & 21.1 & & 7,8 & \\
\hline 13 & $1.31 \mathrm{~s}$ & 18.5 & & $4,2,3$ & \\
\hline 14 & $1.09 \mathrm{~s}$ & 30.8 & 15 & $15,11,10,1$ & \\
\hline 15 & $0.98 \mathrm{~s}$ & 23.5 & 14 & $14,11,10,1$ & \\
\hline OH-C10 & $4.05 \mathrm{~d}(10.7)$ & & 10 & 10,9 & \\
\hline
\end{tabular}

\section{Structure elucidation of compound 5a:}

Compound 5a measured at $\mathrm{m} / \mathrm{z} 275.1618$ by HR-ESI-MS indicates $[\mathrm{M}+\mathrm{Na}]^{+}$ion, suggesting another transannular cyclized product with molecular formula $\mathrm{C}_{15} \mathrm{H}_{24} \mathrm{O}_{3}$. The presence of four $\mathrm{sp}^{2}$ hybridized carbons comprising two quaternary $(\mathrm{C} 7, \mathrm{C} 8)$, two methines (C6, C9), and the absence of a ketone carbonyl suggests compound 5a corresponds to a different core structure (Table S4). The presence of ${ }^{3} J$ COSY correlation of $\mathrm{H}-9\left(\delta_{\mathrm{H}} 5.57 \mathrm{ppm}\right)$ with $\mathrm{H}-10\left(\delta_{\mathrm{H}} 3.62 \mathrm{ppm}\right)$ indicates $\mathrm{C} 10$ as an 
oxygenated methine. The presence of HMBC correlations of $\mathrm{H}-10$ with $\mathrm{C} 1, \mathrm{C} 8, \mathrm{C} 9$, and $\mathrm{H}-9$ with $\mathrm{C} 7, \mathrm{C} 8, \mathrm{C} 10$, reveal the presence of allylic alcohol $\mathrm{C} 10(\mathrm{OH})-\mathrm{C} 9-\mathrm{C} 8$, and $\mathrm{C} 8$ as an oxygenated quaternary carbon due to its most downfield chemical shift of $\delta_{\mathrm{C}} 154.0 \mathrm{ppm}$. The presence of hydroxyl group on $\mathrm{C} 10$ was further evident by the presence of ${ }^{2} J$ COSY and HMBC correlations of hydroxyl proton (Table S4). The presence of HMBC correlations of methyl H-12 with C7, C8, and ${ }^{4} J$ COSY correlation with H-6 $\left(\delta_{\mathrm{H}} 5.80 \mathrm{ppm}\right)$ indicate the partial structure with conjugated double bonds. The presence of ${ }^{3} J$ COSY correlations of H-6 with H-5, H-5 with H-4; and HMBC correlations of $\mathrm{H}-4$ with $\mathrm{C} 2, \mathrm{C} 5, \mathrm{C} 13$, and $\mathrm{H}-13$ with $\mathrm{C} 2, \mathrm{C} 3, \mathrm{C} 4$ indicate the presence of methyl $\mathrm{C} 13$ on oxygenated quaternary carbon $\mathrm{C} 3\left(\delta_{\mathrm{C}} 84.5 \mathrm{ppm}\right)$, which in turn is flanked by methylene $\mathrm{C} 4$ and oxygenated methine $\mathrm{C} 2\left(\delta_{\mathrm{C}} 73.9 \mathrm{ppm}\right)$. Based on the molecular formula, four oxygenated carbons $\mathrm{C} 10, \mathrm{C} 8, \mathrm{C} 3, \mathrm{C} 2$, indicate the presence of two hydroxy groups and one ether linkage. The presence of HMBC correlations of gem-dimethyl $(\mathrm{C} 14, \mathrm{C} 15)$ with methylene $\mathrm{C} 1, \mathrm{C} 10, \mathrm{C} 11\left(4^{\circ}\right)$ indicates the presence of gem-dimethyl groups on $\mathrm{C} 11$ flanked by $\mathrm{C} 1$ and $\mathrm{C} 10$. Finally, the presence of ${ }^{3} J$ COSY correlation of H-2 with H-1 completes the loop of the carbon skeleton. 
Figure S6. HRMS of compound 5a

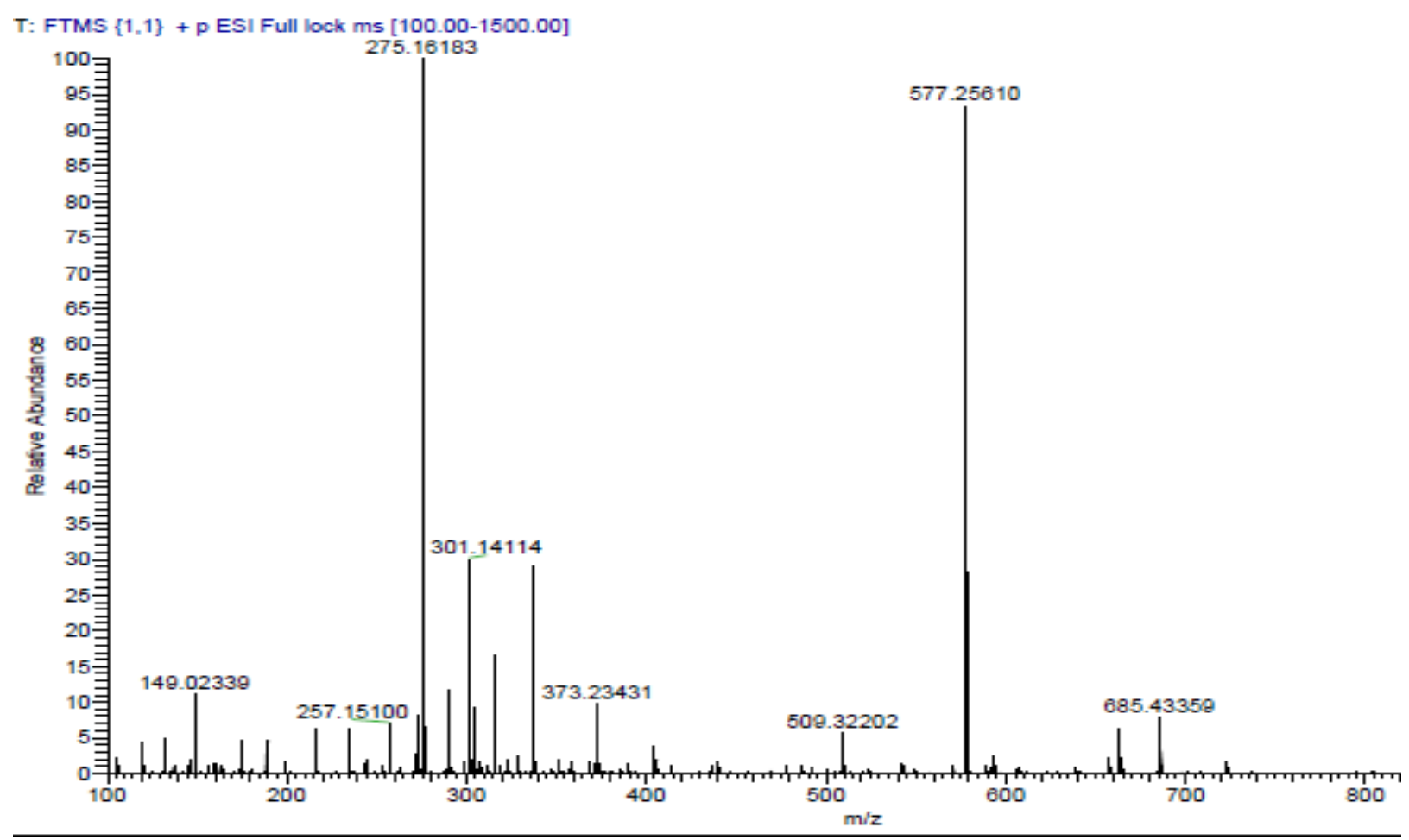

Figure S7. 1D NOE spectrum of compound 5a

14,15

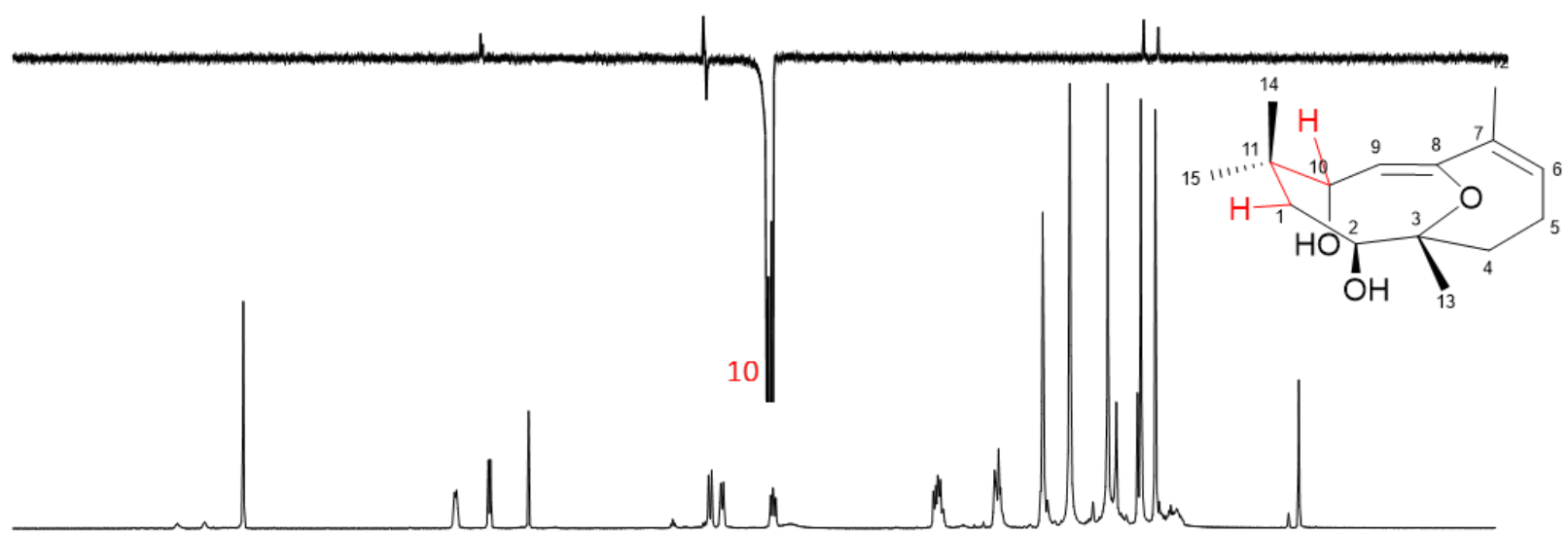

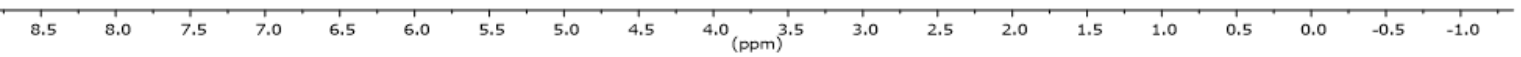


Table S5. ${ }^{1} \mathrm{H}(500 \mathrm{MHz}),{ }^{13} \mathrm{C}(125 \mathrm{MHz}), \mathrm{COSY}$ and $\mathrm{HMBC}$ NMR data of compound $\mathbf{5 b}$ in $\mathrm{CDCl}_{3}$

\begin{tabular}{|c|c|c|c|c|c|}
\hline Position & $\delta_{\mathbf{H}}(J)$ & $\delta \mathrm{C}$ & COSY & HMBC & \\
\hline 1 & $\begin{array}{c}2.20 \mathrm{~m}, \\
1.31 \mathrm{dd}(19.0, \\
4.1)\end{array}$ & 47.2 & 1 & $\begin{array}{c}15,14,2,3,11 \\
10\end{array}$ & 15 \\
\hline 2 & $3.90 \mathrm{~d}(8.7)$ & 73.5 & 1 & $13,11,1,3,4$ & \\
\hline 3 & & 82.4 & & & \\
\hline 4 & $\begin{array}{l}2.05 \mathrm{~m} \\
1.71 \mathrm{~m}\end{array}$ & 42.2 & 4 & $13,5,2,3$ & \\
\hline 5 & $\begin{array}{l}2.50 \mathrm{t}(15.0) \\
2.02(\mathrm{~m})\end{array}$ & 24.0 & 4 & & \\
\hline 6 & 5.79 brd (6.6) & 132.9 & 12,5 & $5,4,8,12$ & \\
\hline 7 & & 132.2 & & & \\
\hline 8 & & 150.8 & & & \\
\hline 9 & $5.11 \mathrm{~d}(5.4)$ & 121.4 & 10 & $10,7,8,11$ & \\
\hline 10 & $4.30 \mathrm{~d}(5.4)$ & 75.7 & & $\begin{array}{c}15,14,11,1, \\
9,8\end{array}$ & \\
\hline 11 & & 38.3 & & & \\
\hline 12 & $1.78 \mathrm{~s}$ & 21.0 & & $6,7,8$ & \\
\hline 13 & $1.25 \mathrm{~s}$ & 18.1 & & $4,2,3$ & \\
\hline 14 & $1.08 \mathrm{~s}$ & 29.1 & & $15,11,10,1$ & \\
\hline 15 & $0.98 \mathrm{~s}$ & 18.2 & & $14,11,10,1$ & \\
\hline
\end{tabular}


Figure S8. HRMS of compound $\mathbf{5 b}$

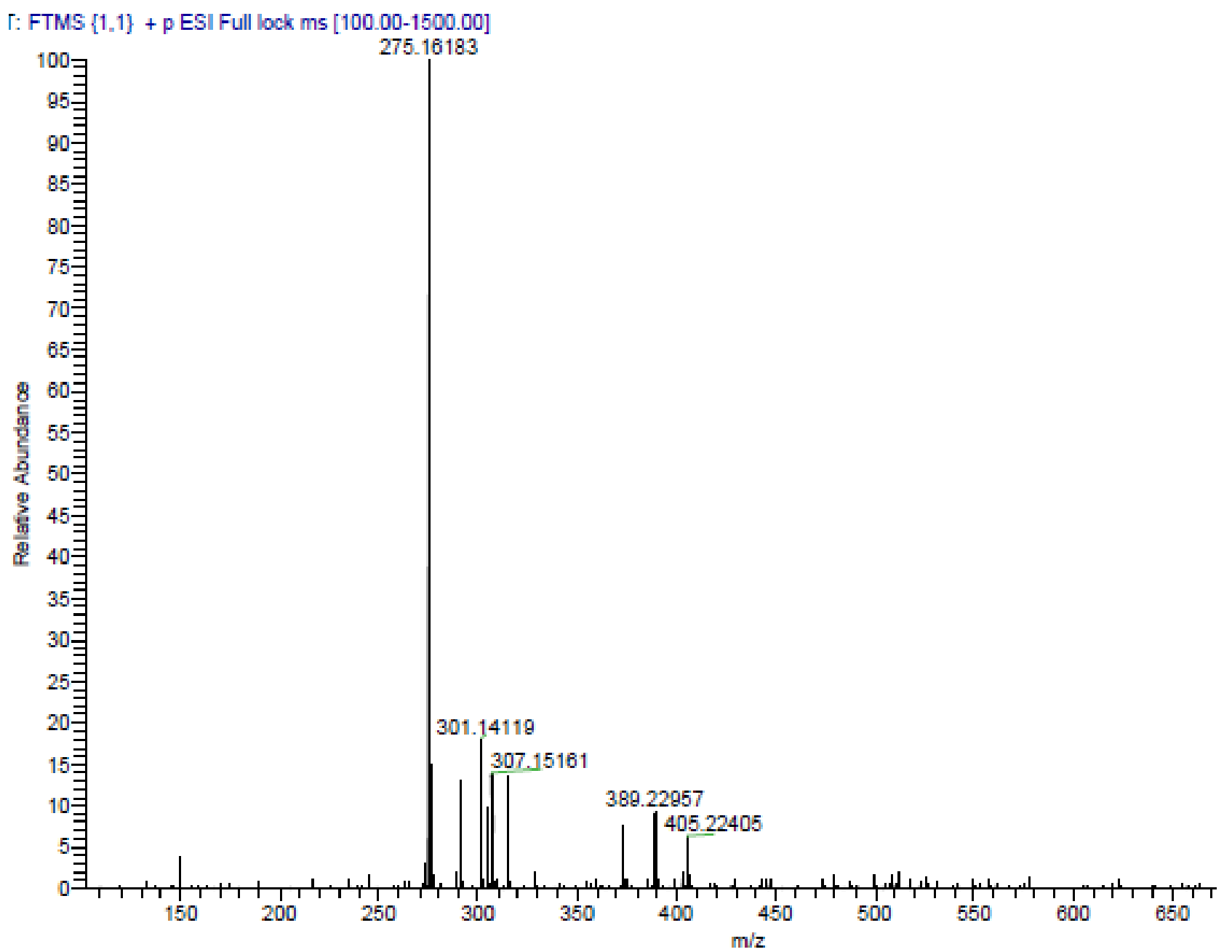


Table S6. ${ }^{1} \mathrm{H}(500 \mathrm{MHz}),{ }^{13} \mathrm{C}(125 \mathrm{MHz}), \mathrm{COSY}$ and $\mathrm{HMBC}$ NMR data of compound $\mathbf{5 c}$ in $\mathrm{CDCl}_{3}$

\begin{tabular}{|c|c|c|c|c|c|}
\hline Position & $\delta_{\mathrm{H}}(\boldsymbol{J})$ & $\delta_{\mathrm{C}}$ & COSY & HMBC & \multirow{16}{*}{ | } \\
\hline 1 & $\begin{array}{l}2.91 \mathrm{~d}(12.3) \\
2.69 \mathrm{~d}(12.4)\end{array}$ & 47.8 & 1 & $14,15,2,10$ & \\
\hline 2 & & 212.8 & & & \\
\hline 3 & & 87.8 & & & \\
\hline 4 & $\begin{array}{l}2.07 \mathrm{~m} \\
1.74 \mathrm{~m}\end{array}$ & 33.8 & 4 & $13,3,5$ & \\
\hline 5 & $\begin{array}{l}2.44 \mathrm{~m} \\
2.18 \mathrm{~m}\end{array}$ & 24.0 & 5,4 & 3,7 & \\
\hline 6 & $5.82 \mathrm{~d}(8.0)$ & 134.7 & 12,5 & 8 & \\
\hline 7 & & 132.0 & & & \\
\hline 8 & & 157.0 & & & \\
\hline 9 & $5.56 \mathrm{~s}$ & 116.6 & & 7,8 & \\
\hline 10 & & 208.4 & & & \\
\hline 11 & & 47.8 & & & \\
\hline 12 & $1.78 \mathrm{~s}$ & 22.2 & & $6,7,8$ & \\
\hline 13 & $1.35 \mathrm{~s}$ & 22.5 & & $4,3,2$ & \\
\hline 14 & $1.12 \mathrm{~s}$ & 27.8 & & $15,1,10$ & \\
\hline 15 & $1.20 \mathrm{~s}$ & 25.4 & & $14,1,10$ & \\
\hline
\end{tabular}


Figure S9. HRMS of compound 5c

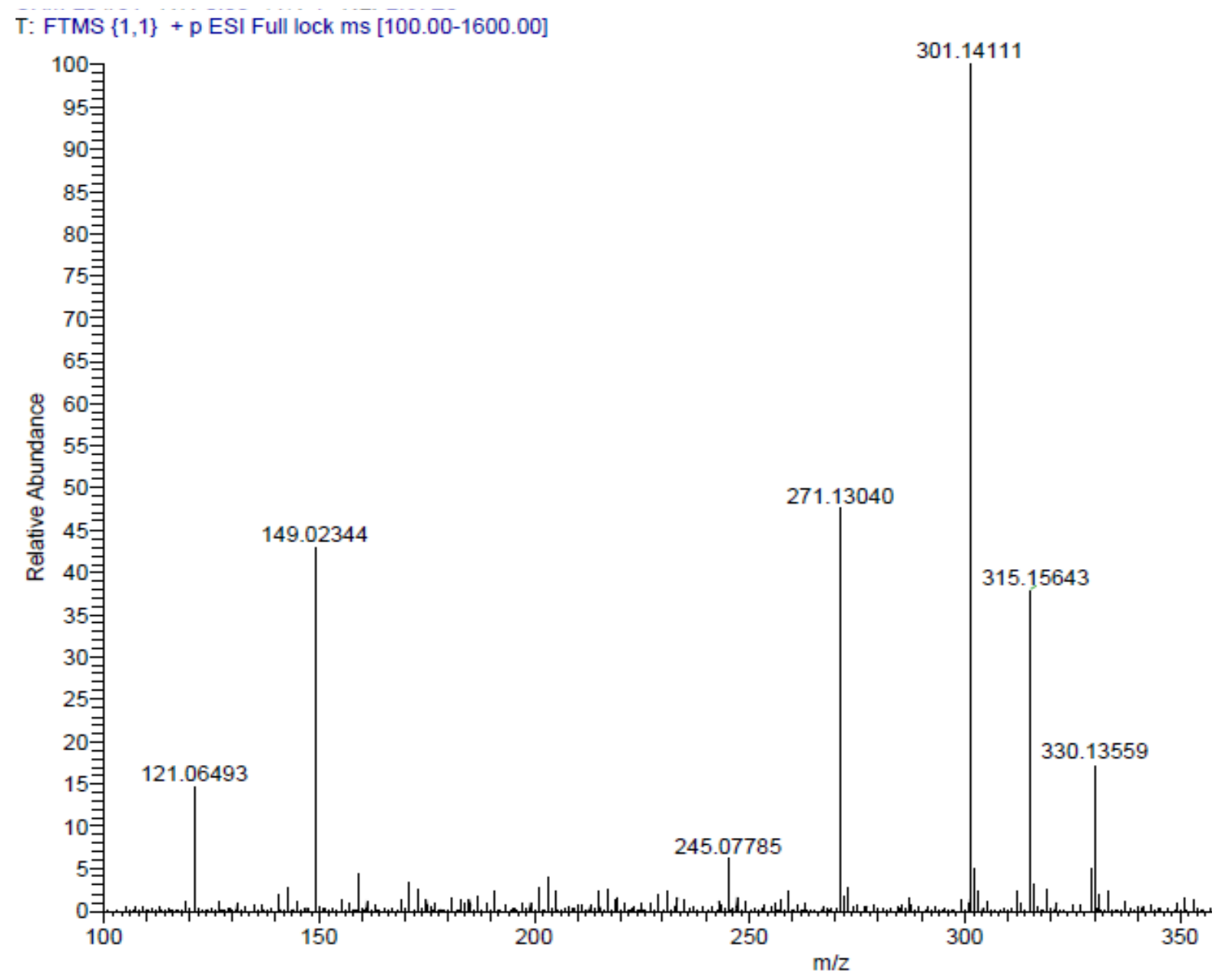


Table S7. ${ }^{1} \mathrm{H}(500 \mathrm{MHz}),{ }^{13} \mathrm{C}(125 \mathrm{MHz}), \mathrm{COSY}$ and $\mathrm{HMBC}$ NMR data of compound 6a in $\mathrm{CDCl}_{3}$

\begin{tabular}{|c|c|c|c|c|c|}
\hline Position & $\delta_{\mathrm{H}}(J)$ & $\delta \mathrm{C}$ & COSY & HMBC & \\
\hline 1 & $2.19 \mathrm{~m}, 1.29 \mathrm{~m}$ & 47.8 & 1 & $2,11,3,14$ & \\
\hline 2 & $3.92 \mathrm{~d}(8.5)$ & 73.5 & 1 & $13,11,4,3$ & \\
\hline 3 & & 82.5 & & & \\
\hline 4 & $2.07 \mathrm{~m}, 1.72 \mathrm{~m}$ & 42.3 & 4 & 13, 2,3, & \\
\hline 5 & $\begin{array}{c}2.51 \mathrm{t}(14.5) \\
2.03 \mathrm{~m}\end{array}$ & 24.0 & 4 & & \\
\hline 6 & 5.79 brd (6.6) & 132.7 & 12,5 & 8,4 & \\
\hline 7 & & 132.4 & & & \\
\hline 8 & & 152.5 & & & \\
\hline 9 & $5.12 \mathrm{~d}(5.5)$ & 121.2 & 10 & $7,8,10$ & \\
\hline 10 & $3.72 \mathrm{~d}(5.5)$ & 85.0 & & $\begin{array}{c}14,11,16 \\
8,9\end{array}$ & \\
\hline 11 & & 38.2 & & & \\
\hline 12 & $1.79 \mathrm{~s}$ & 21.1 & & 7,8 & \\
\hline 13 & $1.26 \mathrm{~s}$ & 18.2 & & $4,2,3$ & \\
\hline 14 & $0.96 \mathrm{~s}$ & 19.1 & & $\begin{array}{c}10,1,11 \\
15\end{array}$ & \\
\hline 15 & $1.06 \mathrm{~s}$ & 29.2 & & $\begin{array}{c}10,1,11 \\
14\end{array}$ & \\
\hline 16 & $3.27 \mathrm{~s}$ & 57.4 & & 10 & \\
\hline
\end{tabular}


Figure S10. HRMS of compound $6 \mathbf{a}$

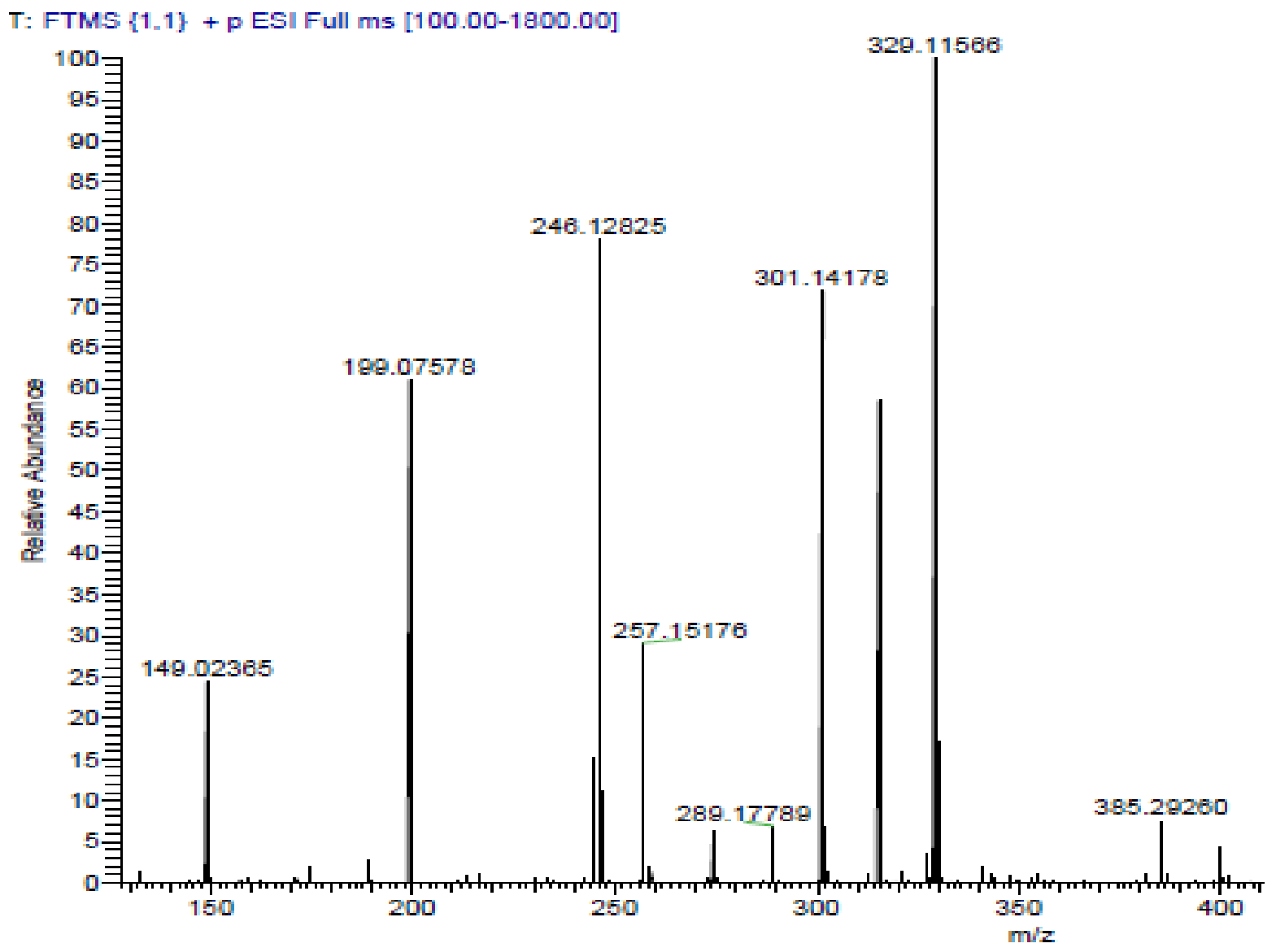


Table S8. ${ }^{1} \mathrm{H}(500 \mathrm{MHz}),{ }^{13} \mathrm{C}(125 \mathrm{MHz}), \mathrm{COSY}$ and $\mathrm{HMBC}$ NMR data of compound $\mathbf{6 b}$ in $\mathrm{CDCl}_{3}$

\begin{tabular}{|c|c|c|c|c|}
\hline Position & $\boldsymbol{\delta} \mathbf{H}(\boldsymbol{J})$ & $\boldsymbol{\delta} \mathbf{C}$ & $\mathbf{C O S Y}$ & HMBC \\
\hline $\mathbf{1}$ & $\begin{array}{c}2.12 \mathrm{dd}(14.9, \\
8.8) \\
1.22 \mathrm{~m}\end{array}$ & 47.8 & 1 & $11,2,3,15$, \\
\hline $\mathbf{2}$ & $3.85 \mathrm{~d}(8.6)$ & 73.5 & 1 & $13,11,1$, \\
\hline $\mathbf{3}$ & & 82.4 & & \\
\hline $\mathbf{4}$ & $1.97 \mathrm{~m}, 1.64 \mathrm{~m}$ & 42.3 & 4 & $3,5,2,13$ \\
\hline $\mathbf{5}$ & $2.43 \mathrm{~m}, 1.99 \mathrm{~m}$ & 24.0 & 4,5 & \\
\hline $\mathbf{6}$ & $5.70 \mathrm{~d}(7.4)$ & 132.4 & 5,12 & \\
\hline $\mathbf{7}$ & & 128.9 & & \\
\hline $\mathbf{8}$ & & 151.8 & & \\
\hline $\mathbf{9}$ & $5.07 \mathrm{~d}(5.3)$ & 122.0 & 10 & 10,8 \\
\hline $\mathbf{1 0}$ & $3.74 \mathrm{~d}(5.0)$ & 82.9 & & $8,9,16,1$, \\
\hline $\mathbf{1 1}$ & & 38.2 & & 11,15 \\
\hline $\mathbf{1 2}$ & $1.71 \mathrm{~s}$ & 21.1 & & 8,6, \\
\hline $\mathbf{1 3}$ & $1.18 \mathrm{~s}$ & 18.2 & & $4,2,3$ \\
\hline $\mathbf{1 4}$ & $0.99 \mathrm{~s}$ & 29.3 & & $14,11,1,10$ \\
\hline $\mathbf{1 5}$ & $0.89 \mathrm{~s}$ & 19.2 & & $15,11,1,10$ \\
\hline $\mathbf{1 6}$ & $3.49 \mathrm{~m}, 3.22 \mathrm{~m}$ & 64.9 & 16,17 & 10 \\
\hline $\mathbf{1 7}$ & $1.07 \mathrm{t}(7.6)$ & 15.3 & & 16 \\
\hline & & & & \\
\hline & & & \\
\hline
\end{tabular}


Figure S11. HRMS of compound $\mathbf{6 b}$

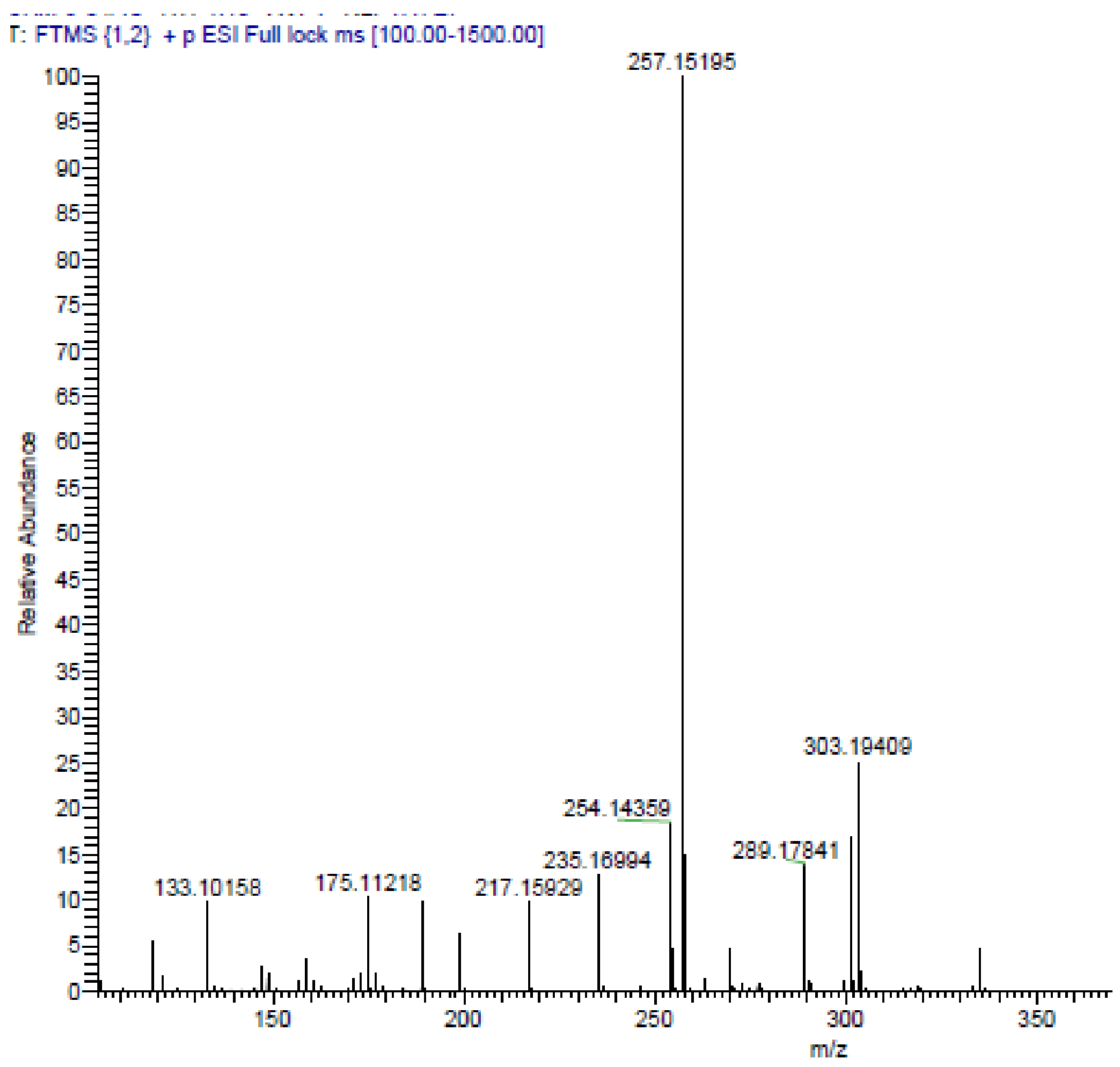


Figure S12. NMR spectra of compound 2

$\mathrm{CD} 3 \operatorname{COCD} 3,500 \mathrm{MHz}$

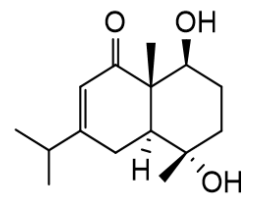

2
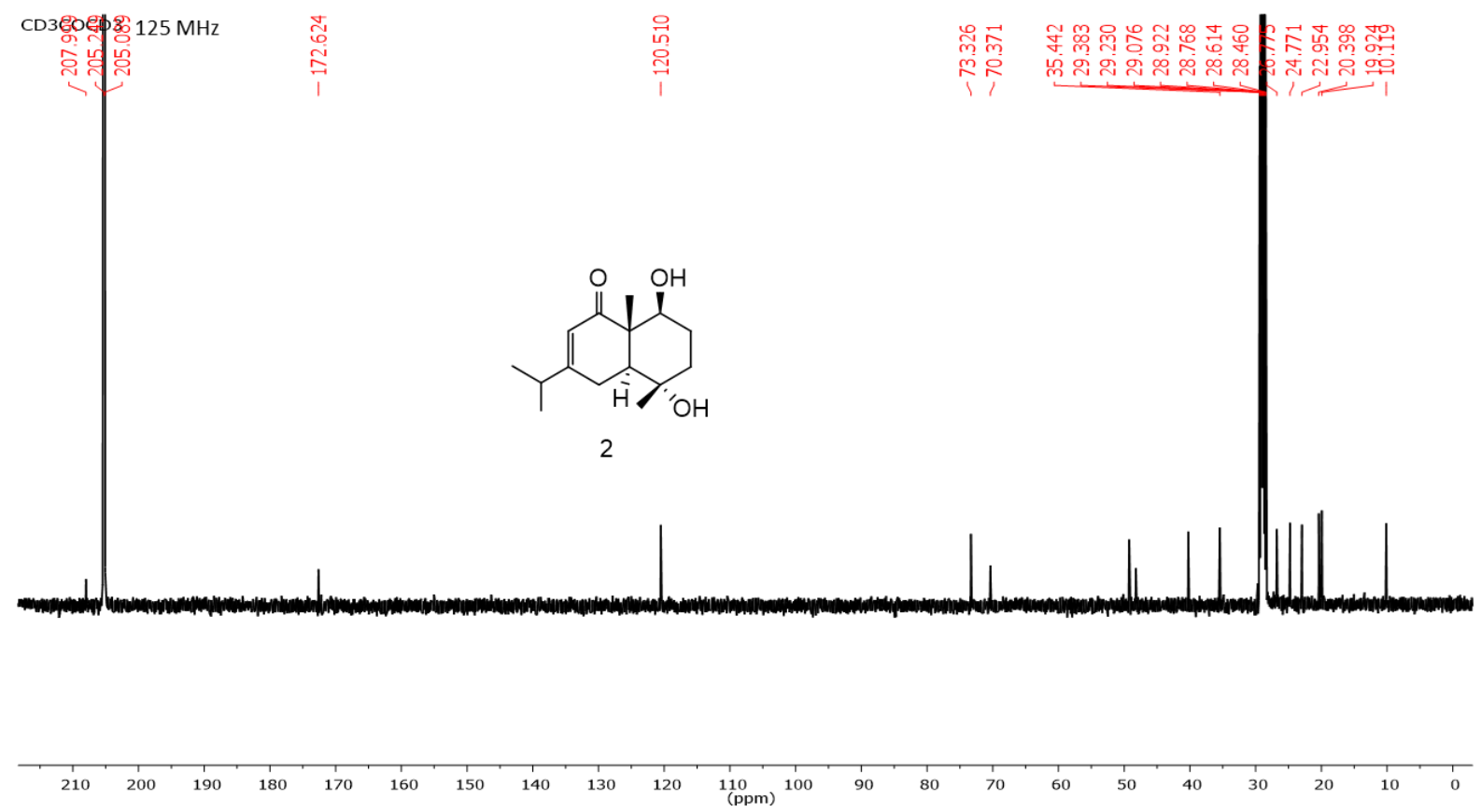


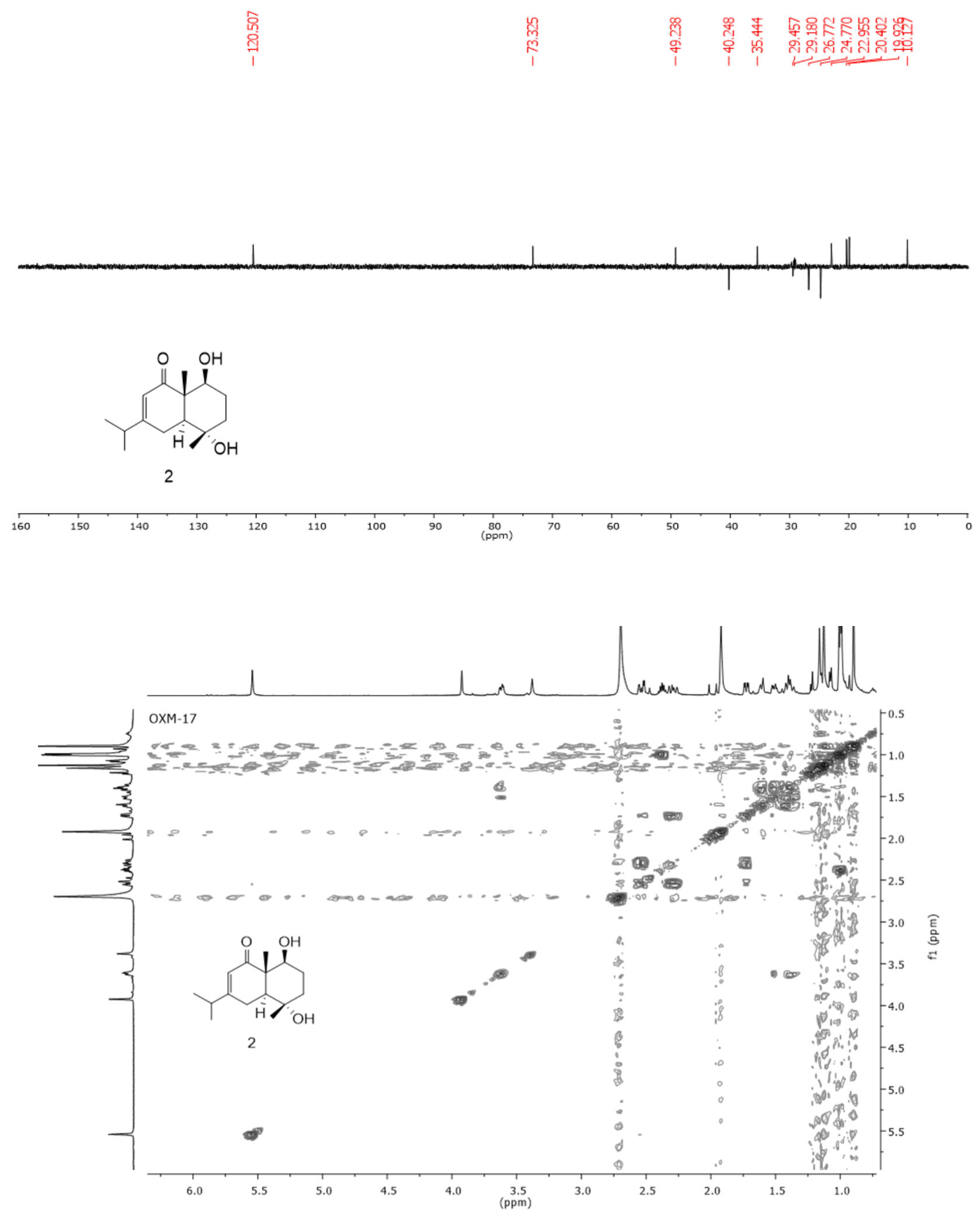



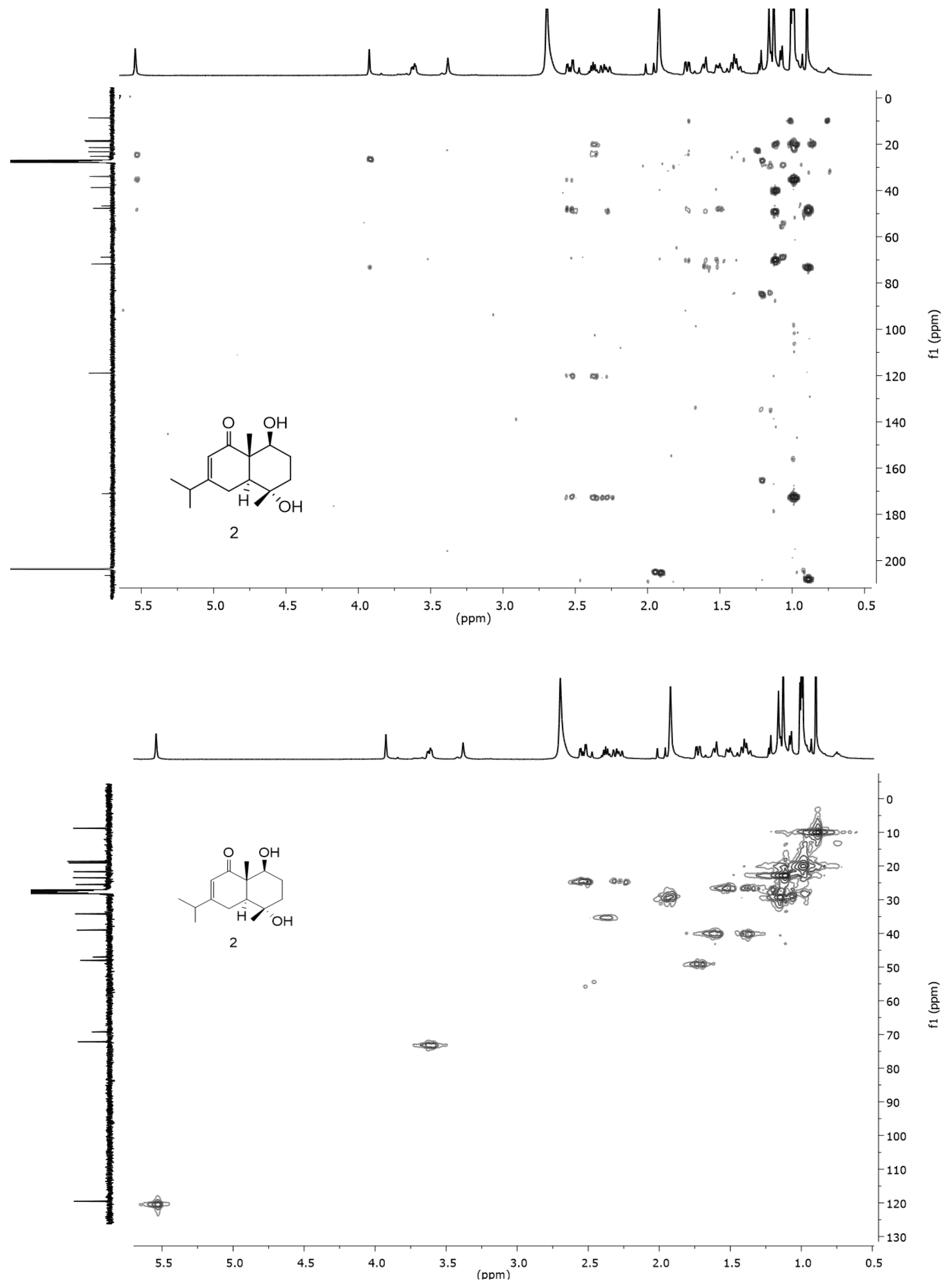
Figure S13. NMR spectra of compound 3
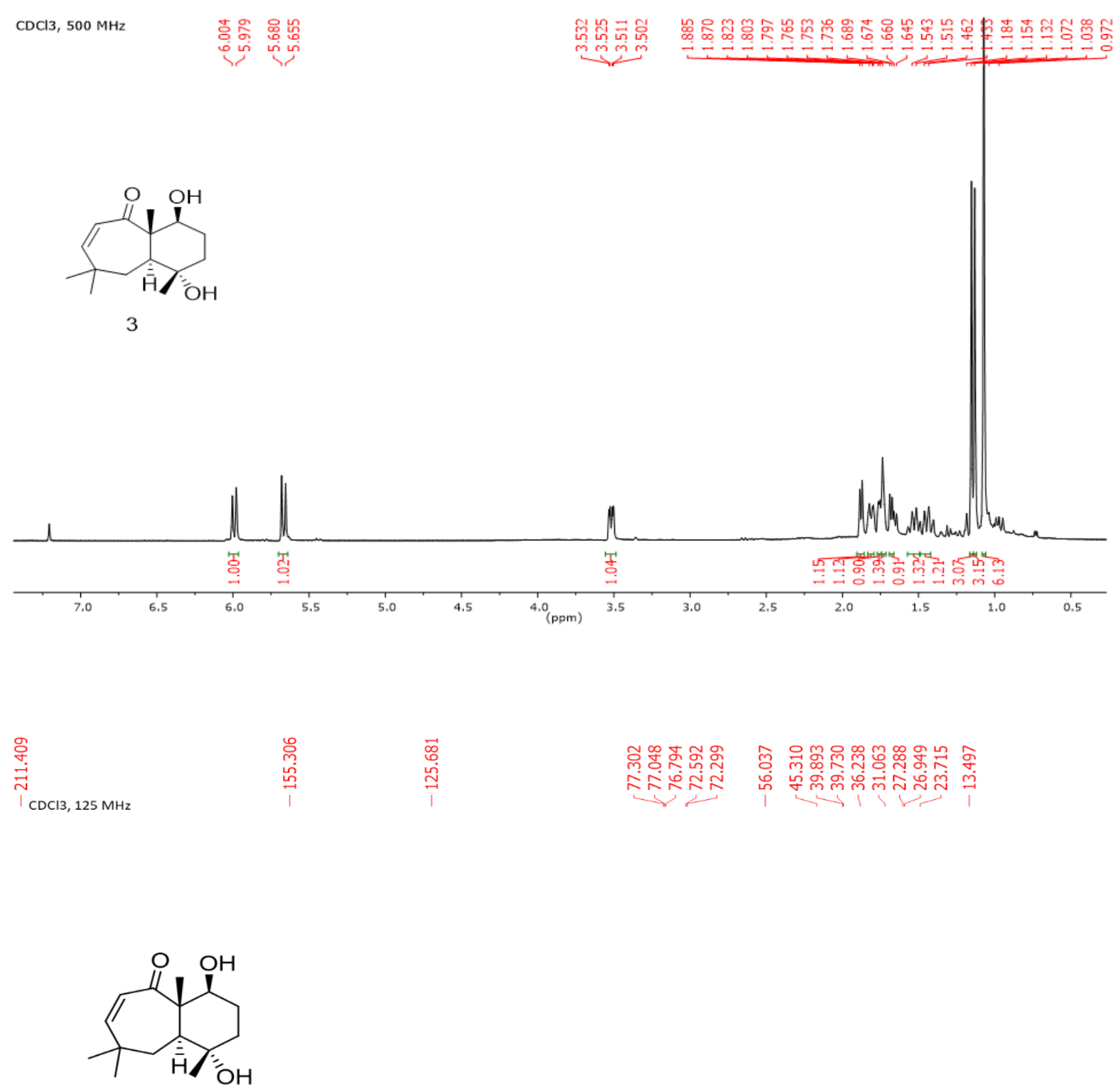

3

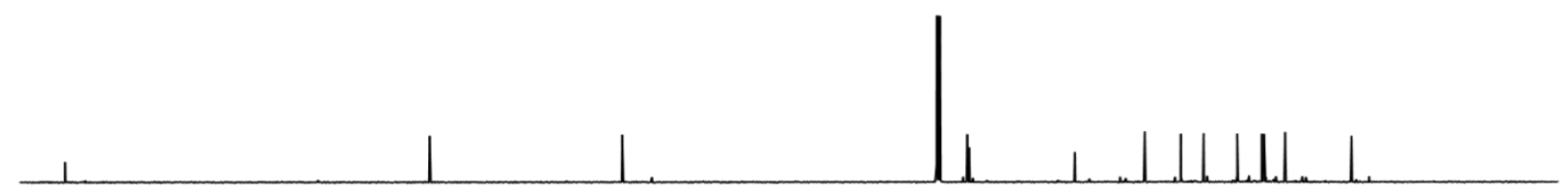

$\begin{array}{llllllllllllllllllllllllllll} & 210 & 200 & 190 & 180 & 170 & 160 & 150 & 140 & 130 & 120 & 110 & 100 & 90 & 80 & 70 & 60 & 50 & 40 & 30 & 20 & 10 & 0 & -10\end{array}$ 


蒏
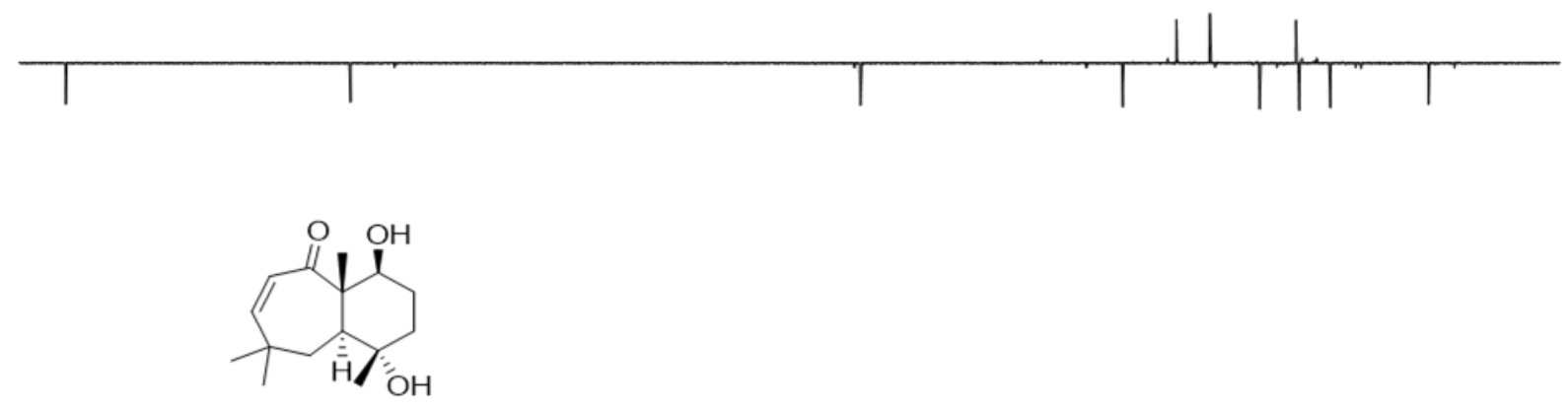

3

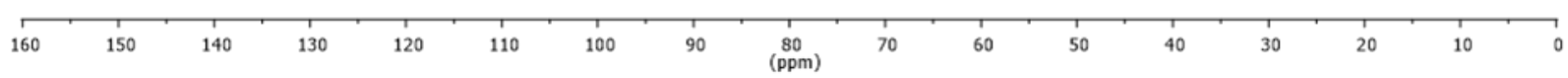

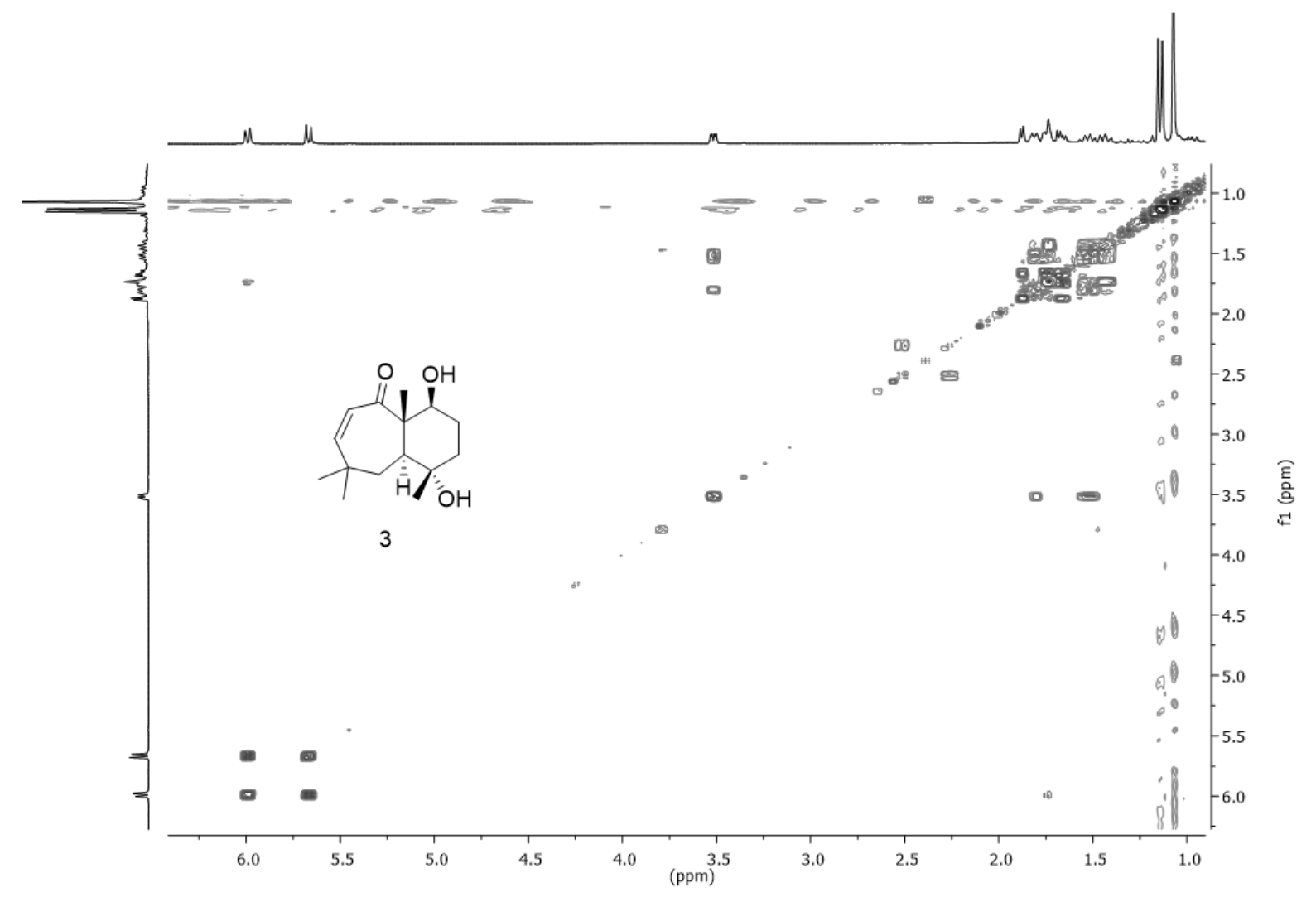



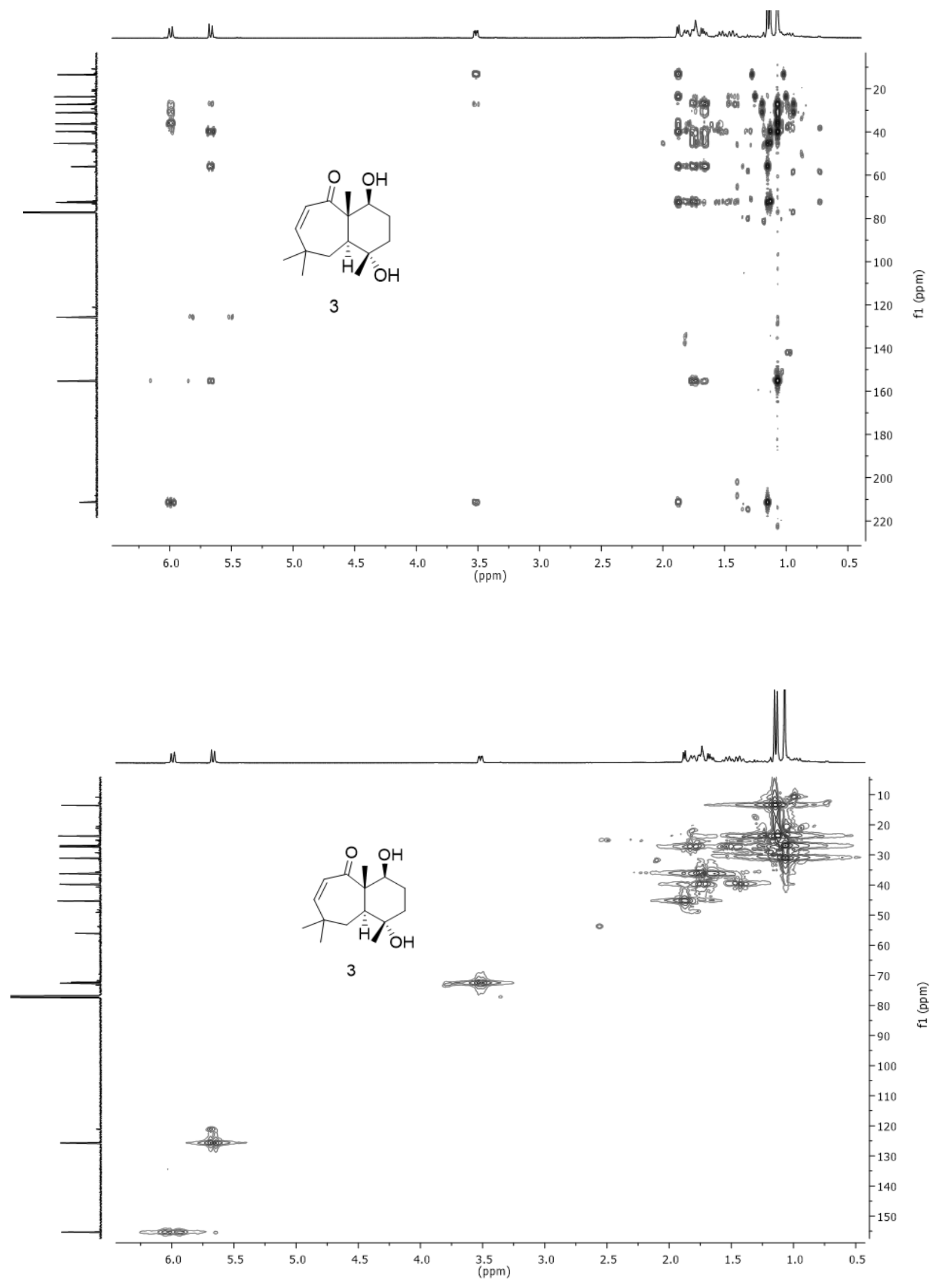
Figure S14. NMR spectra of compound 4
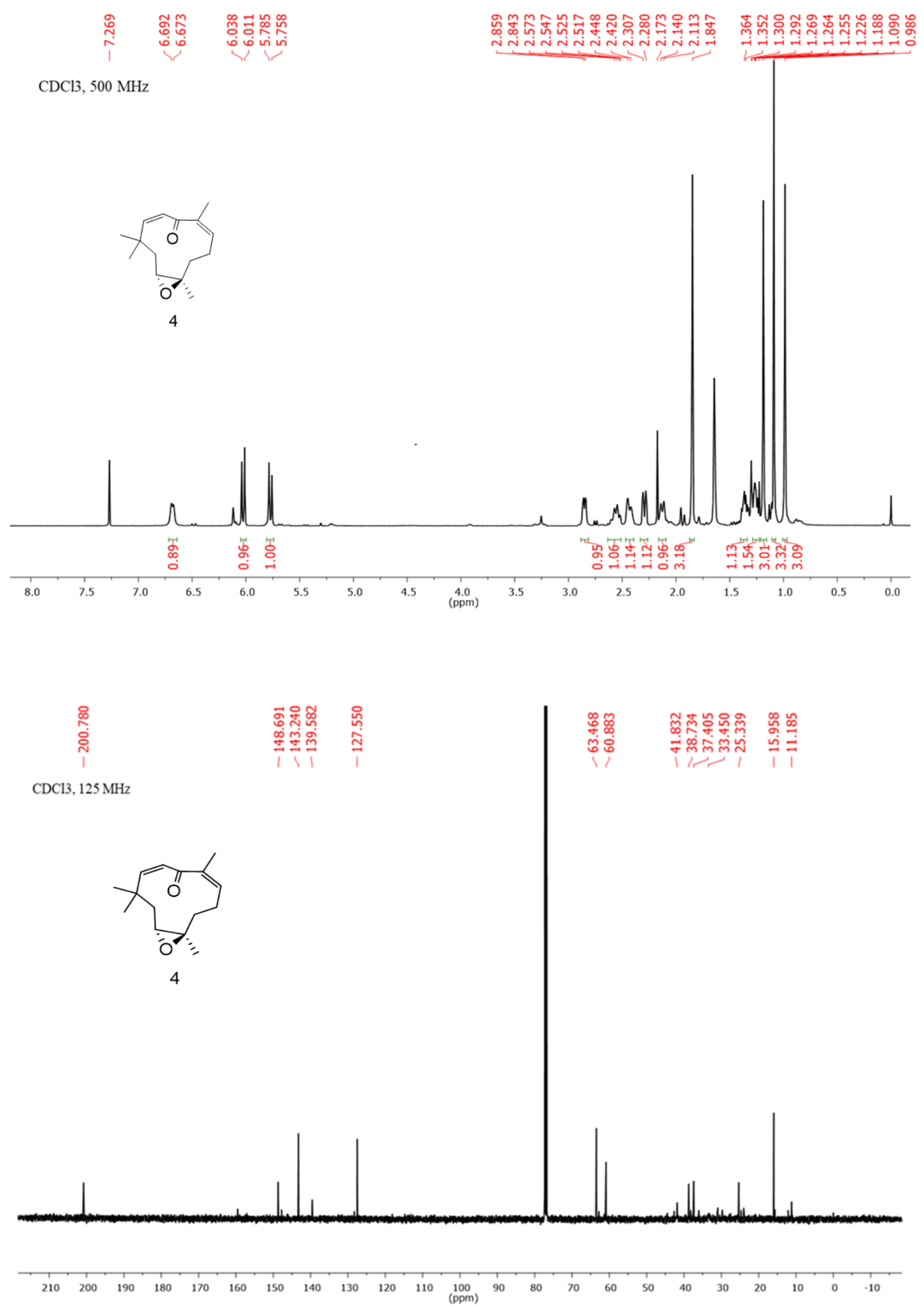


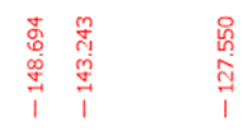
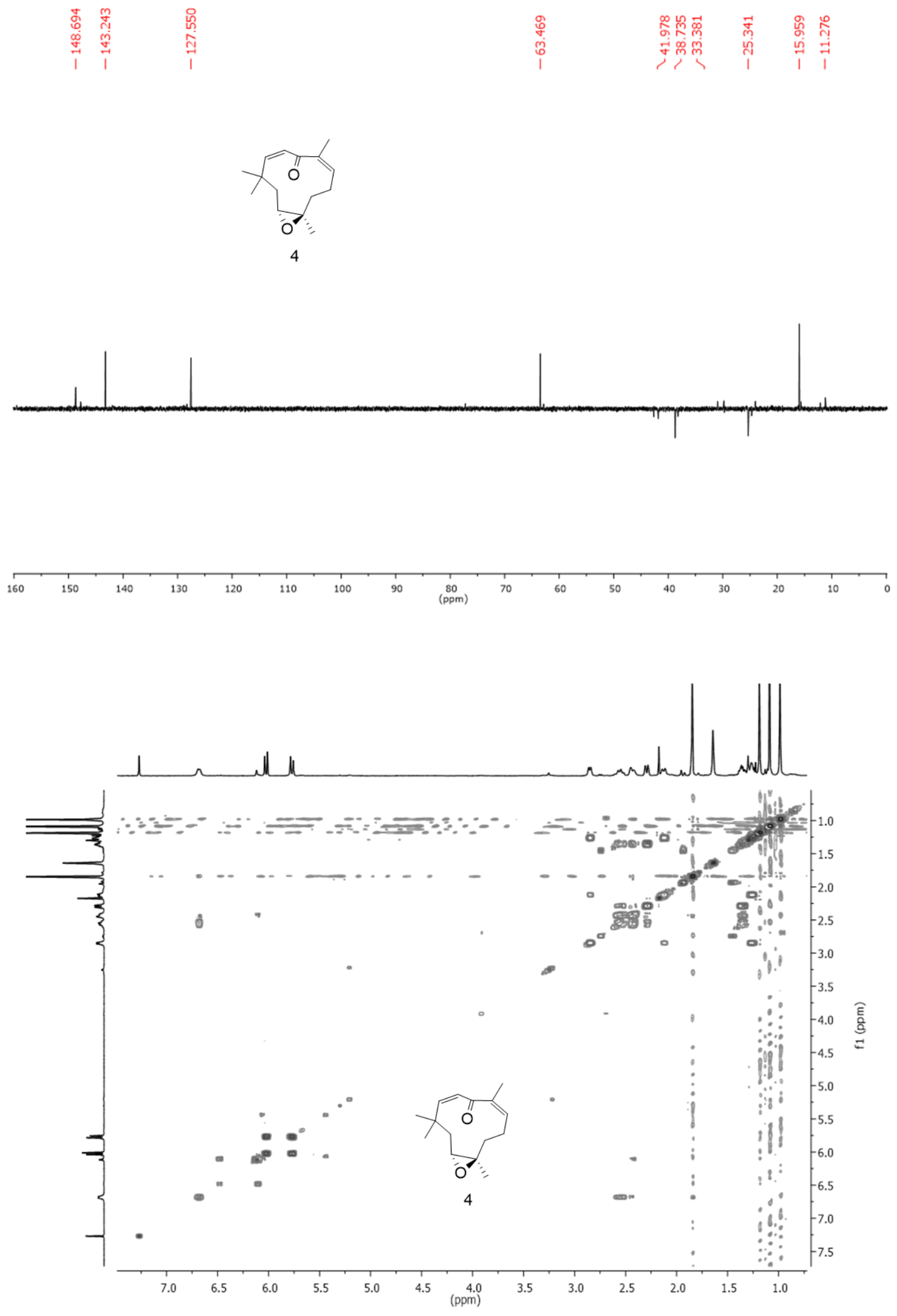

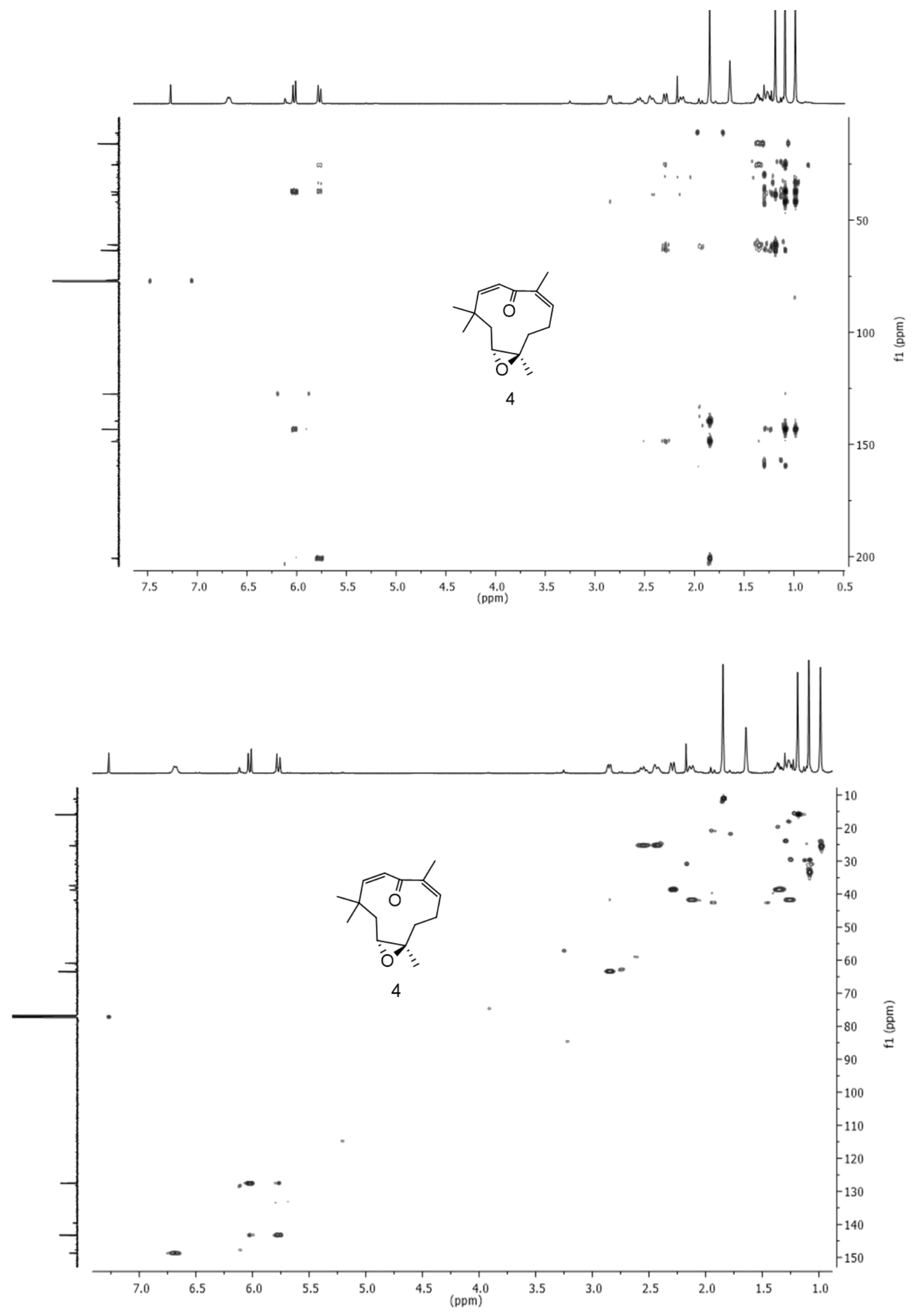
Figure S15. NMR spectra of compound 5a
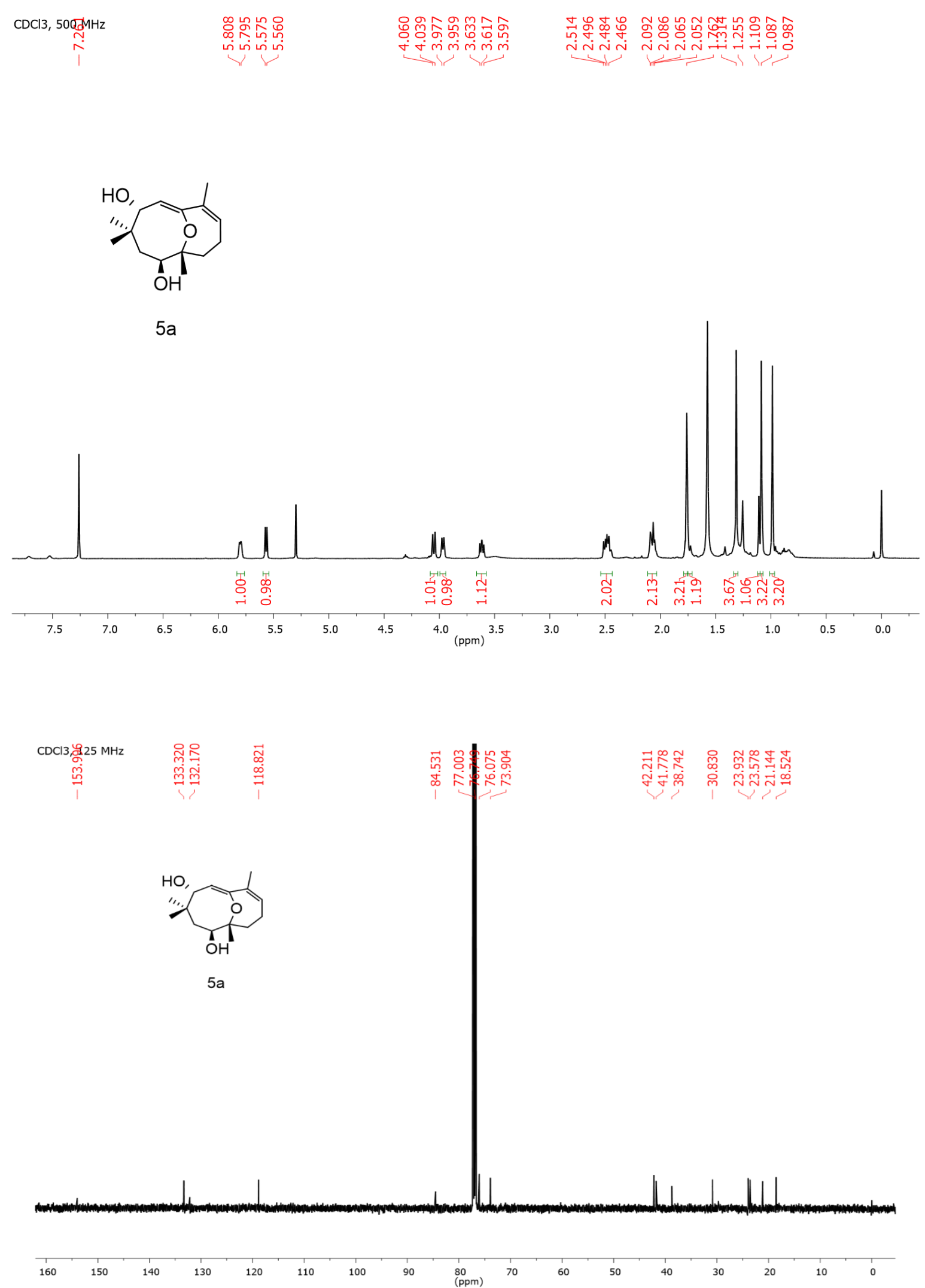


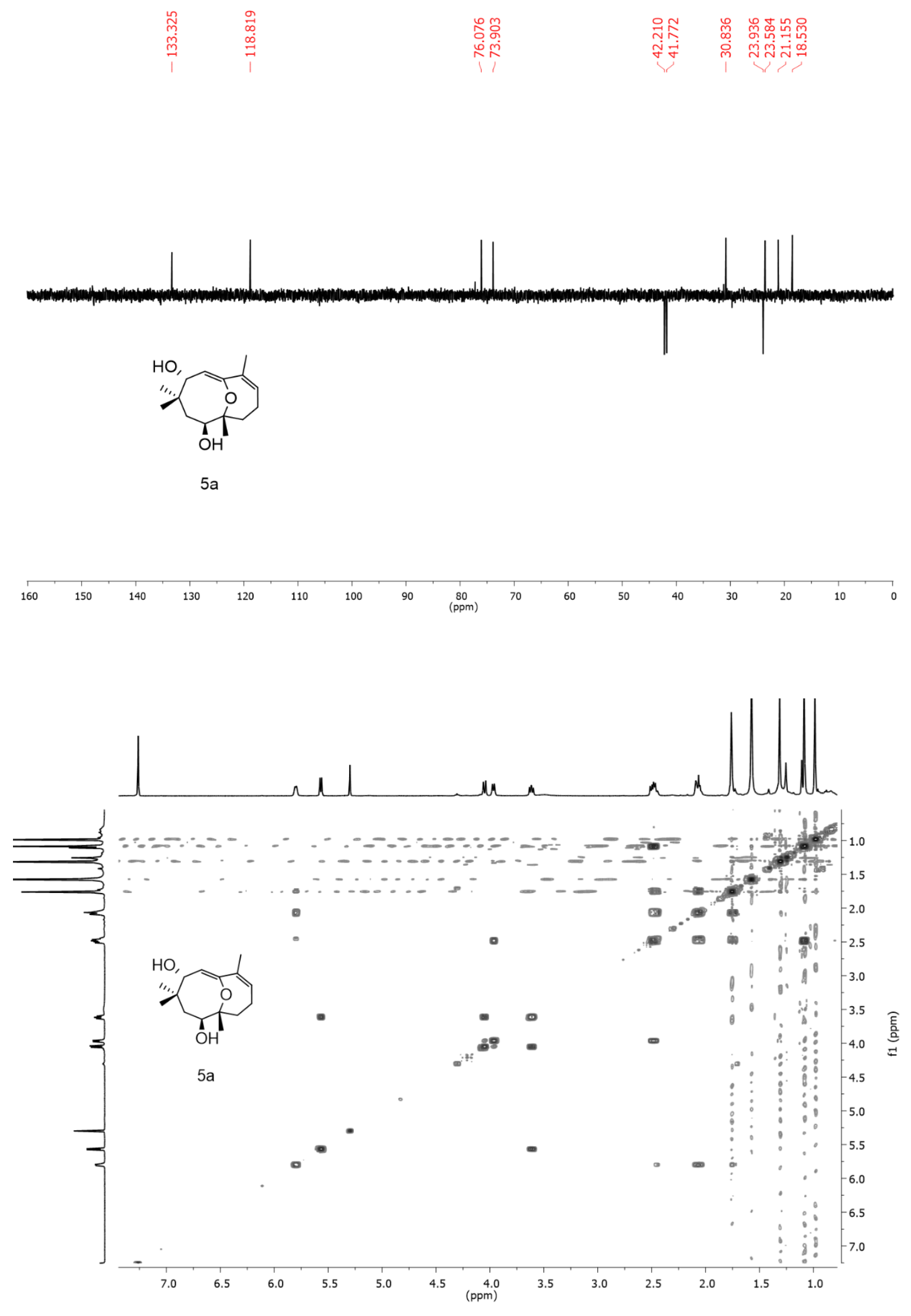



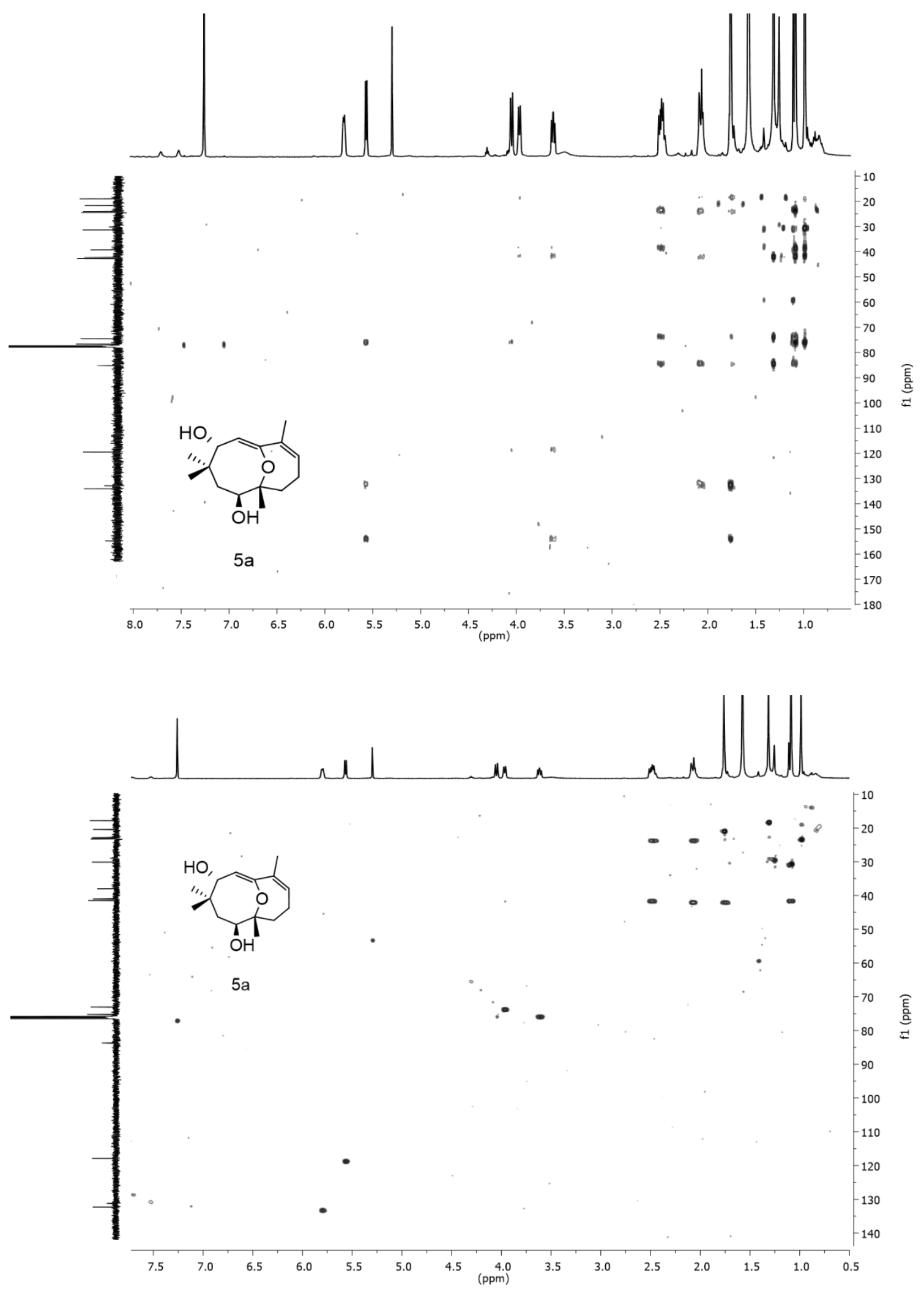
Figure S16. NMR spectra of compound $\mathbf{5 b}$
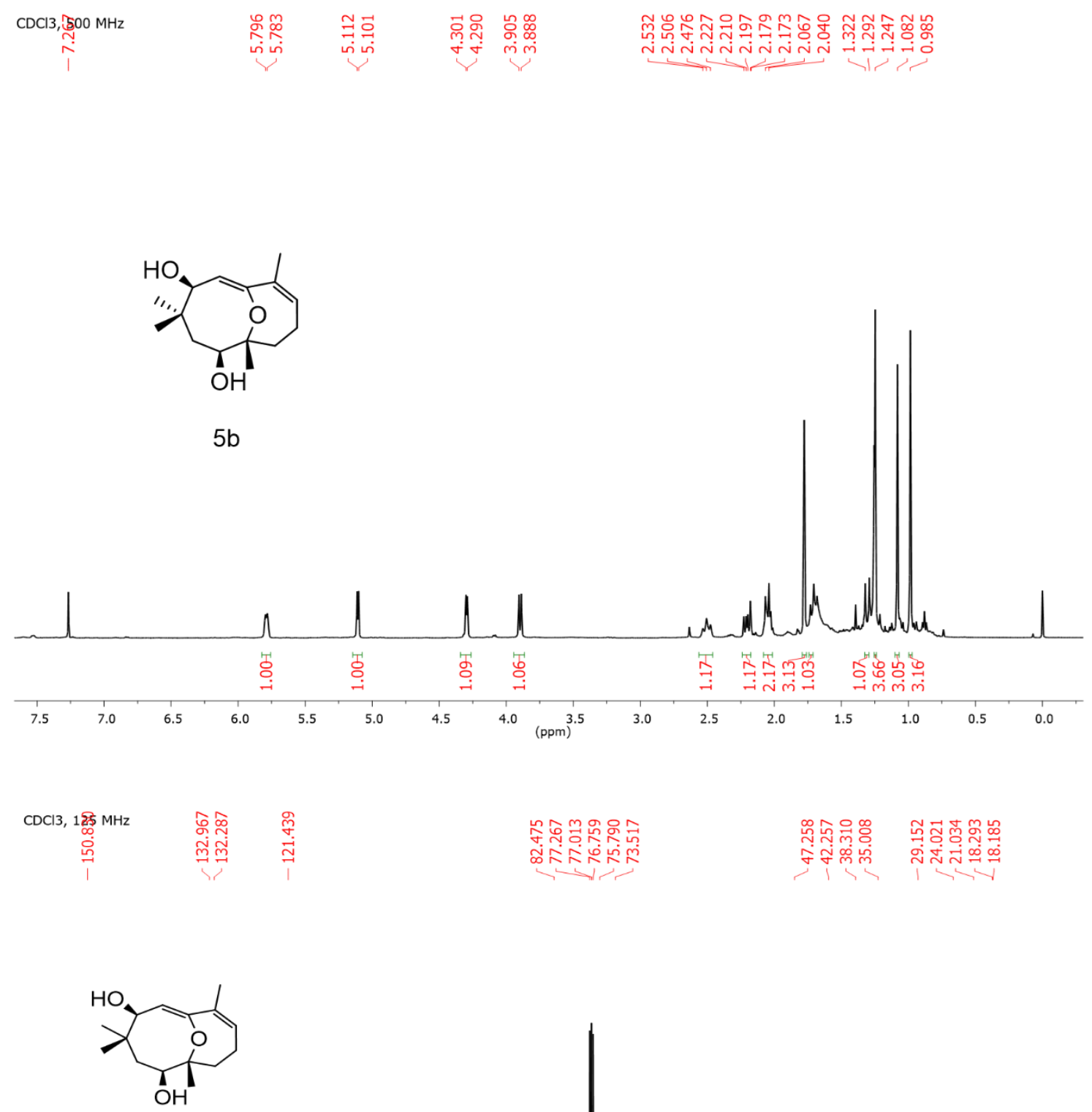

$5 b$
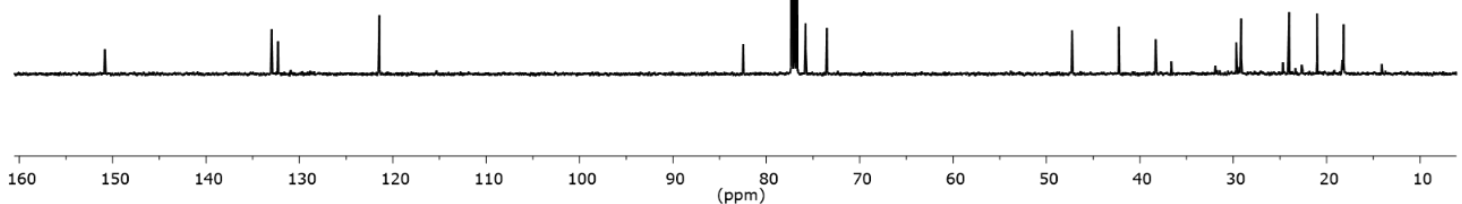


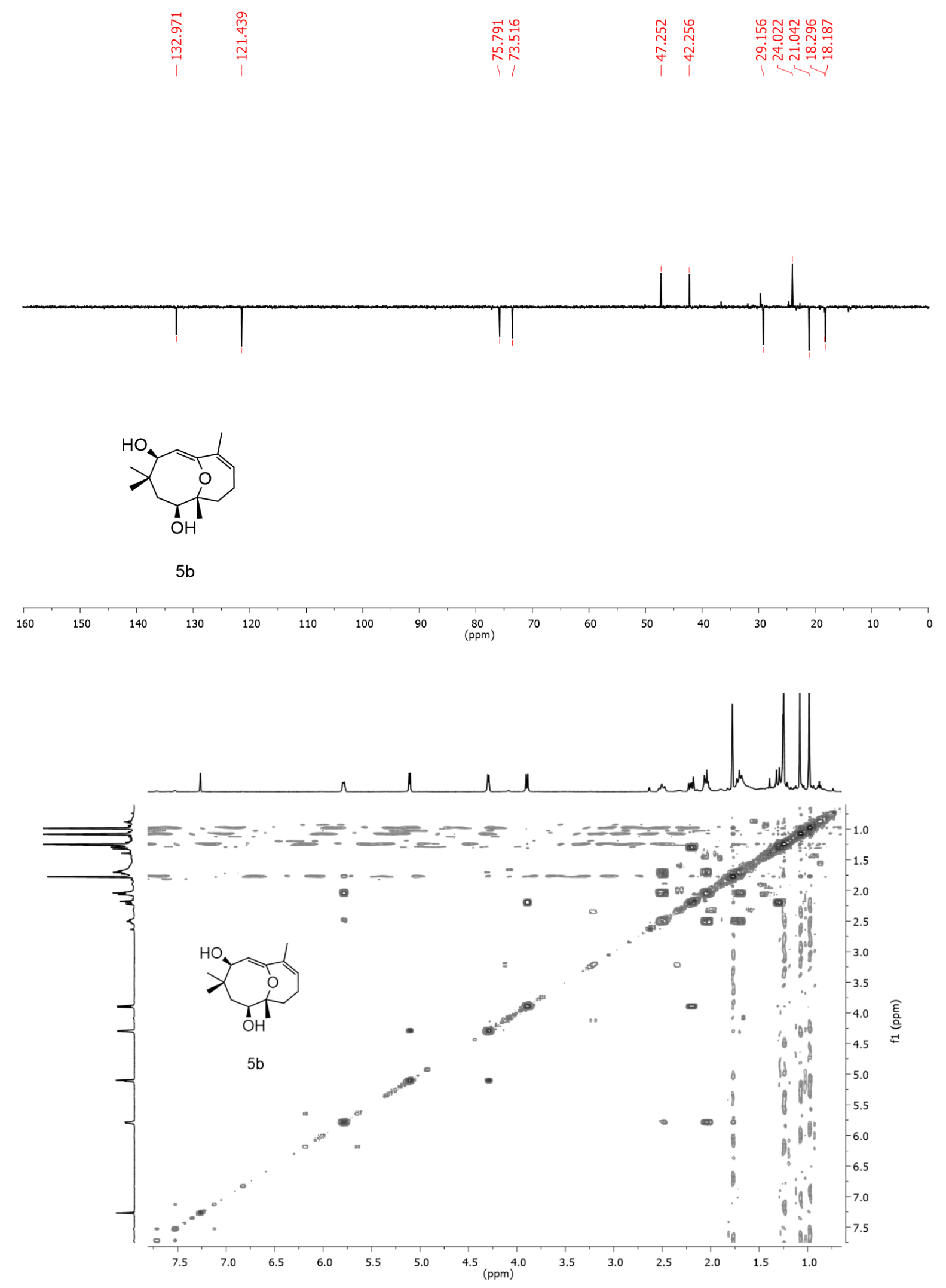



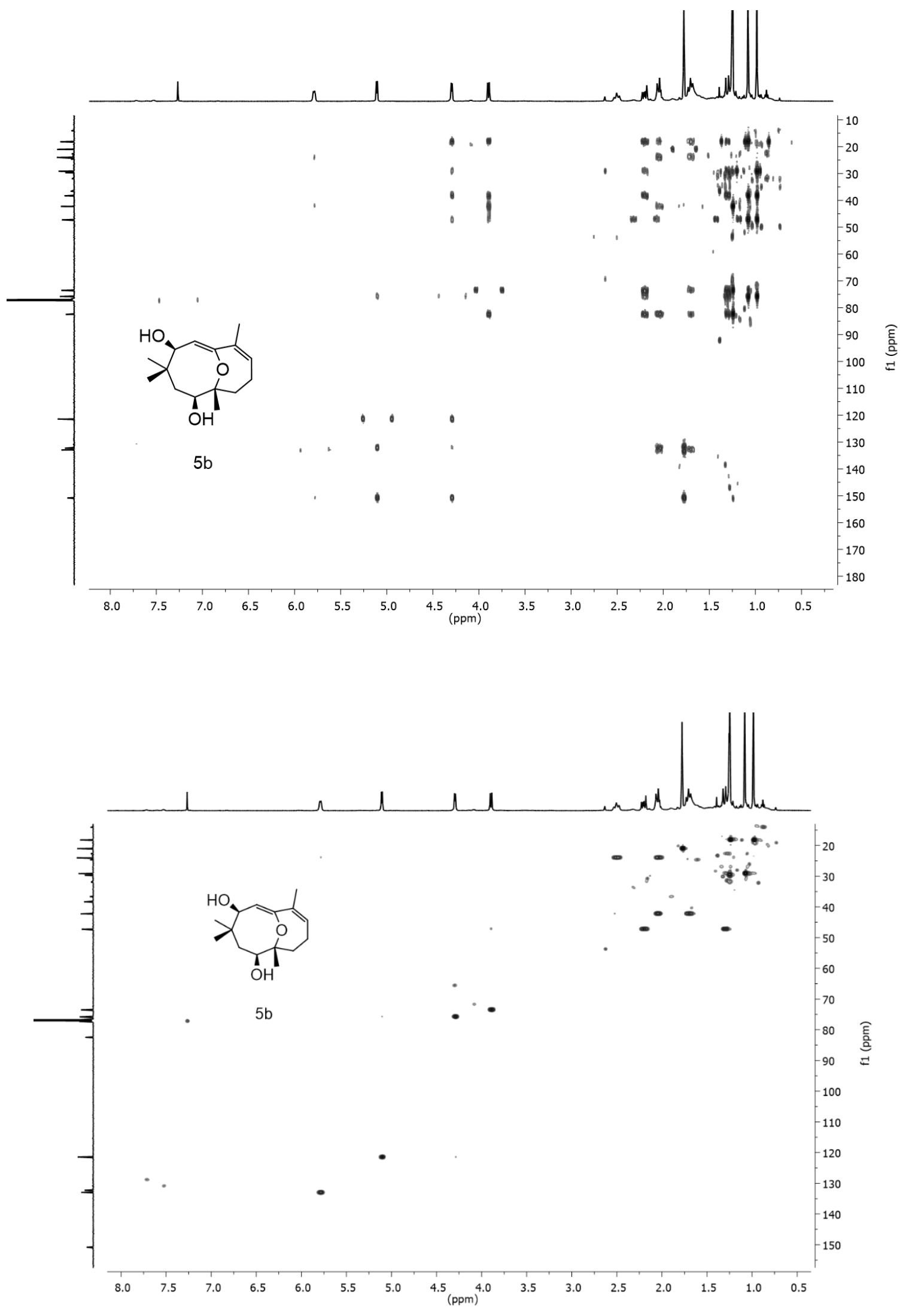
Figure S17. NMR spectra of compound 5c
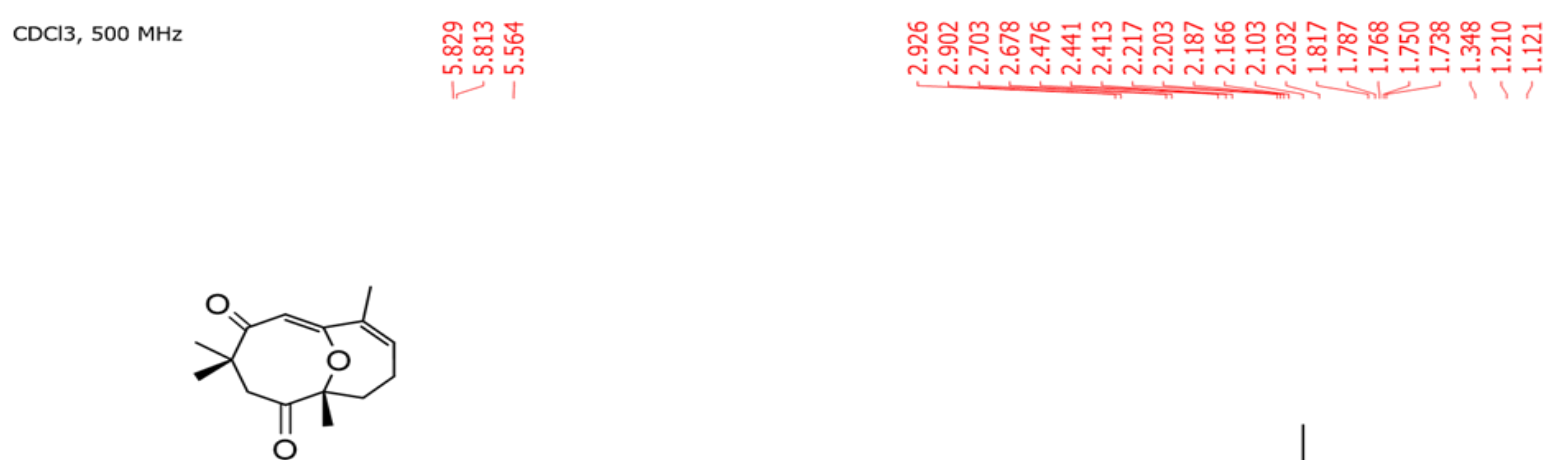

$5 c$

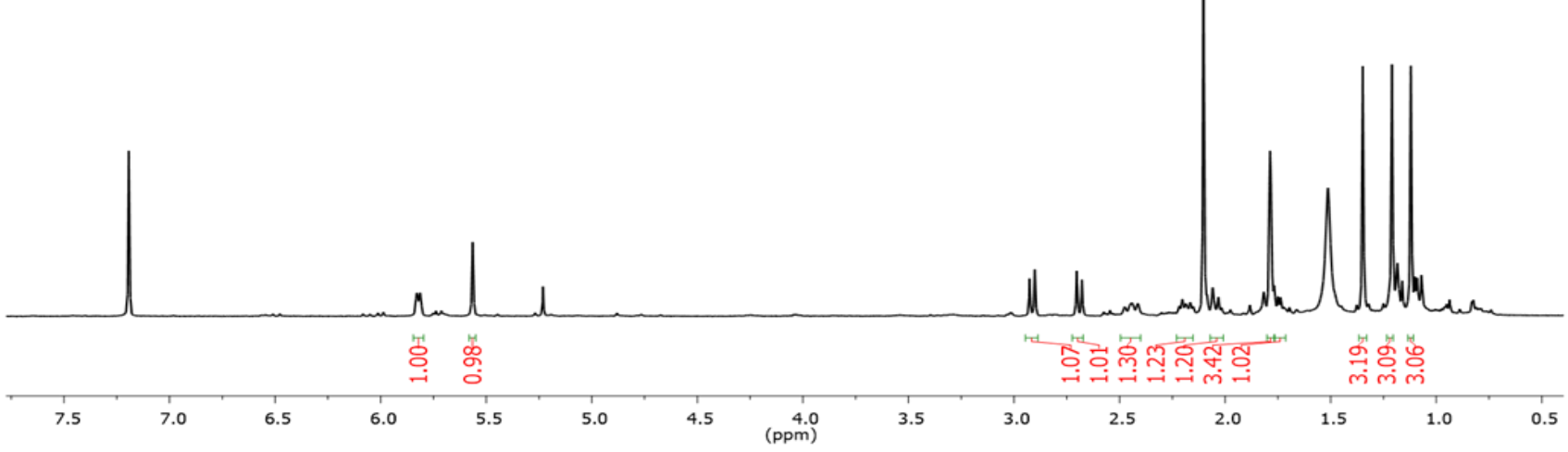

\begin{tabular}{|c|c|c|c|c|c|c|}
\hline 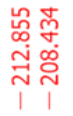 & 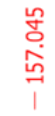 & 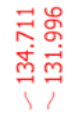 & $\begin{array}{l}\stackrel{m}{\forall} \\
\underset{\sigma}{\sigma} \\
\underset{1}{1}\end{array}$ & $\underset{\substack{\infty \\
\infty}}{\infty}$ & $\begin{array}{c}\text { ָू } \\
\stackrel{\infty}{\tilde{f}} \\
\text { । }\end{array}$ & 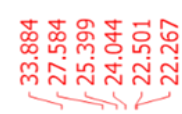 \\
\hline
\end{tabular}

$\mathrm{CDC1}, 125 \mathrm{MHz}$

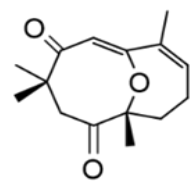

$5 c$

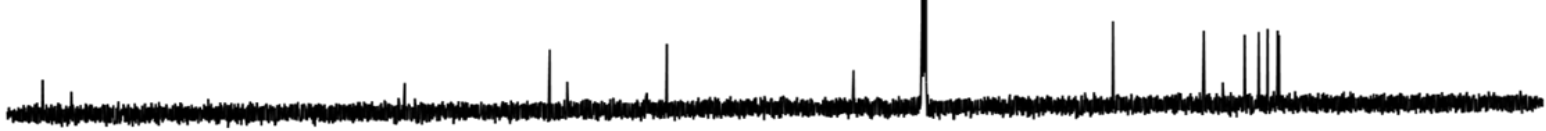

$\begin{array}{llllllllllllllllllllllll}210 & 200 & 190 & 180 & 170 & 160 & 150 & 140 & 130 & 120 & 110 & 100 & 90 & 80 & 70 & 60 & 50 & 40 & 30 & 20 & 10 & 0 & -10\end{array}$ 

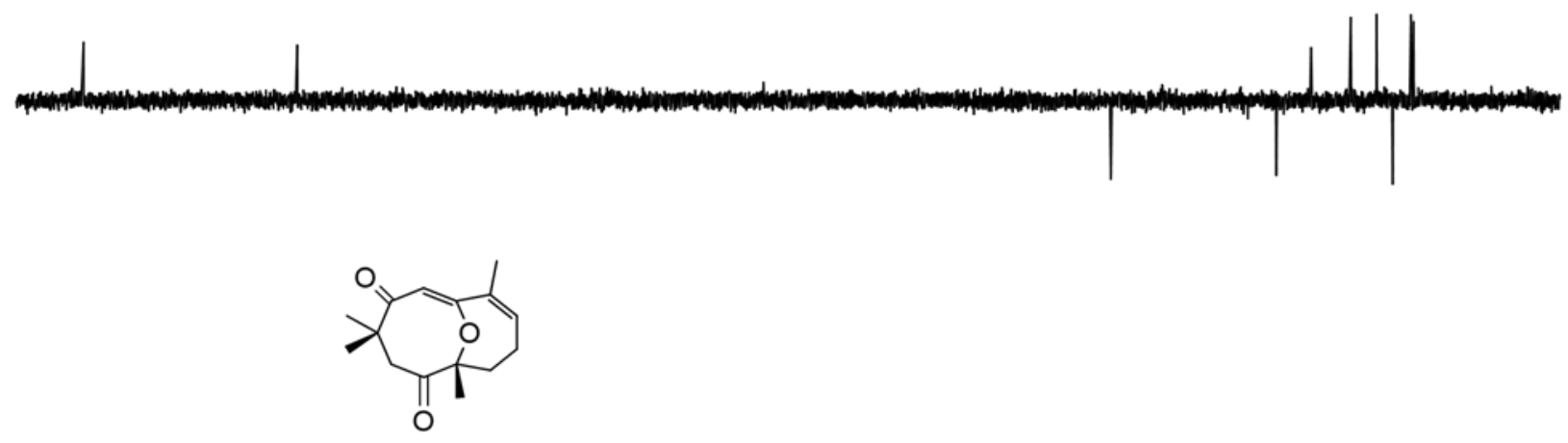

$5 c$
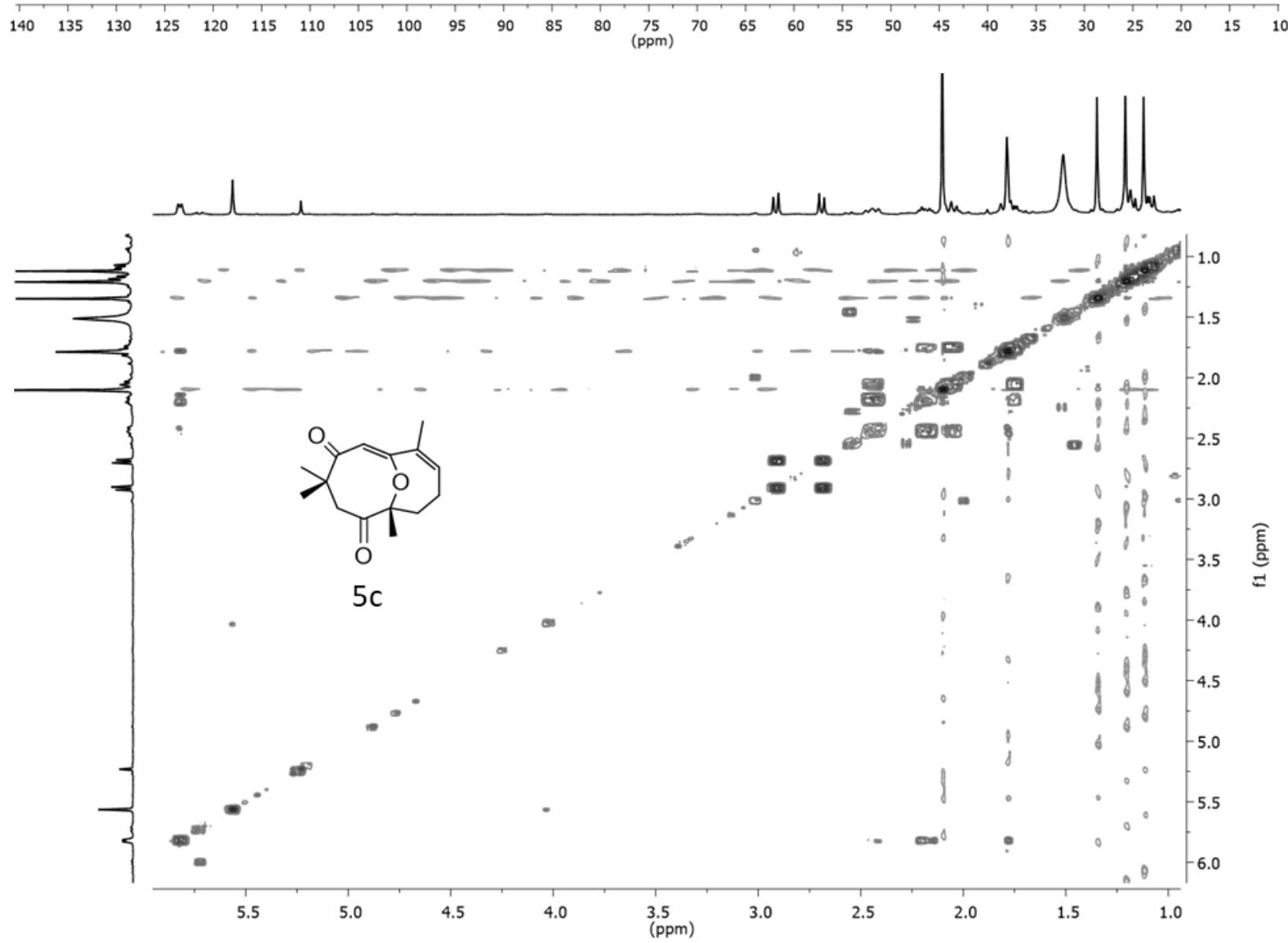

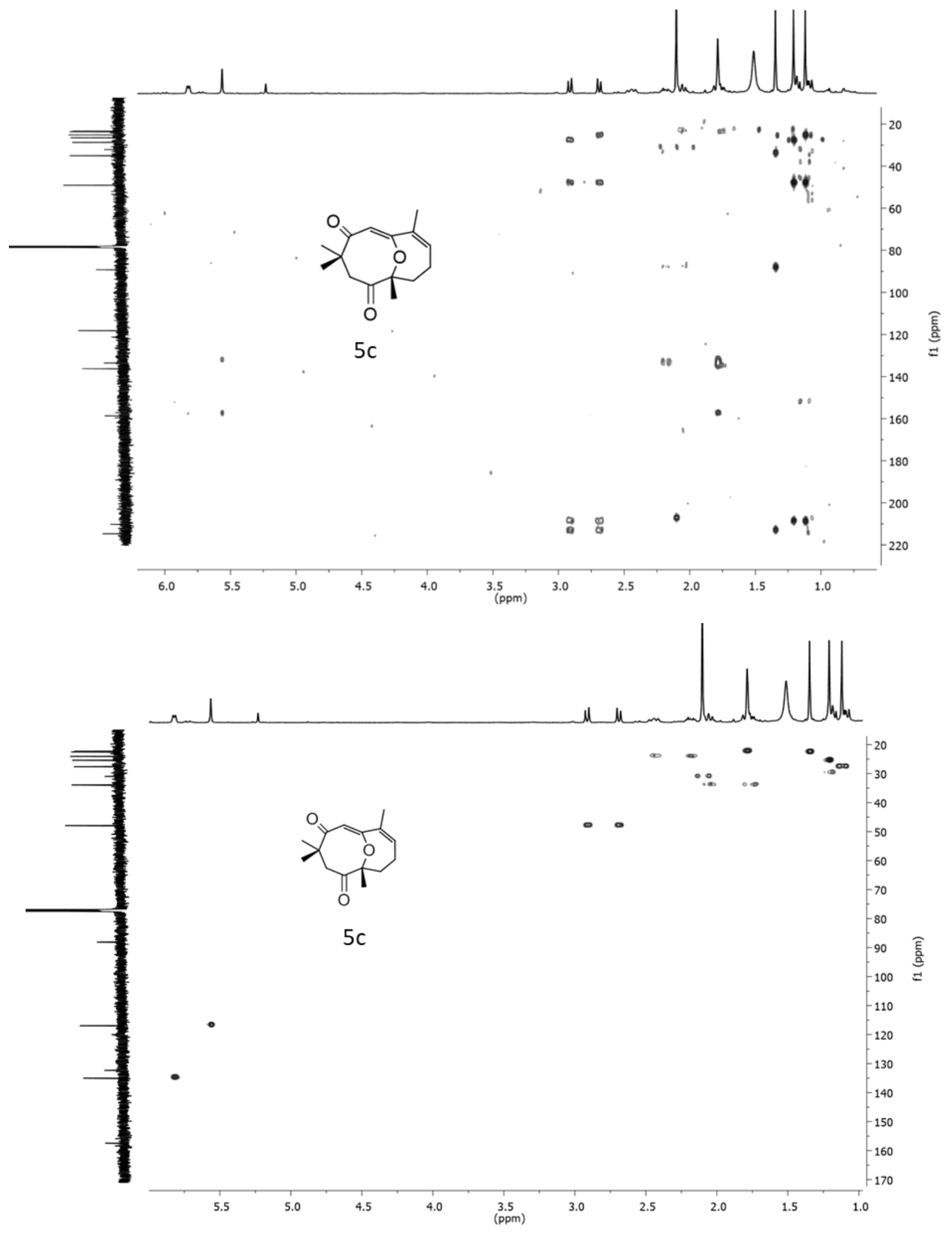
Figure S18. NMR spectra of compound $6 \mathbf{a}$
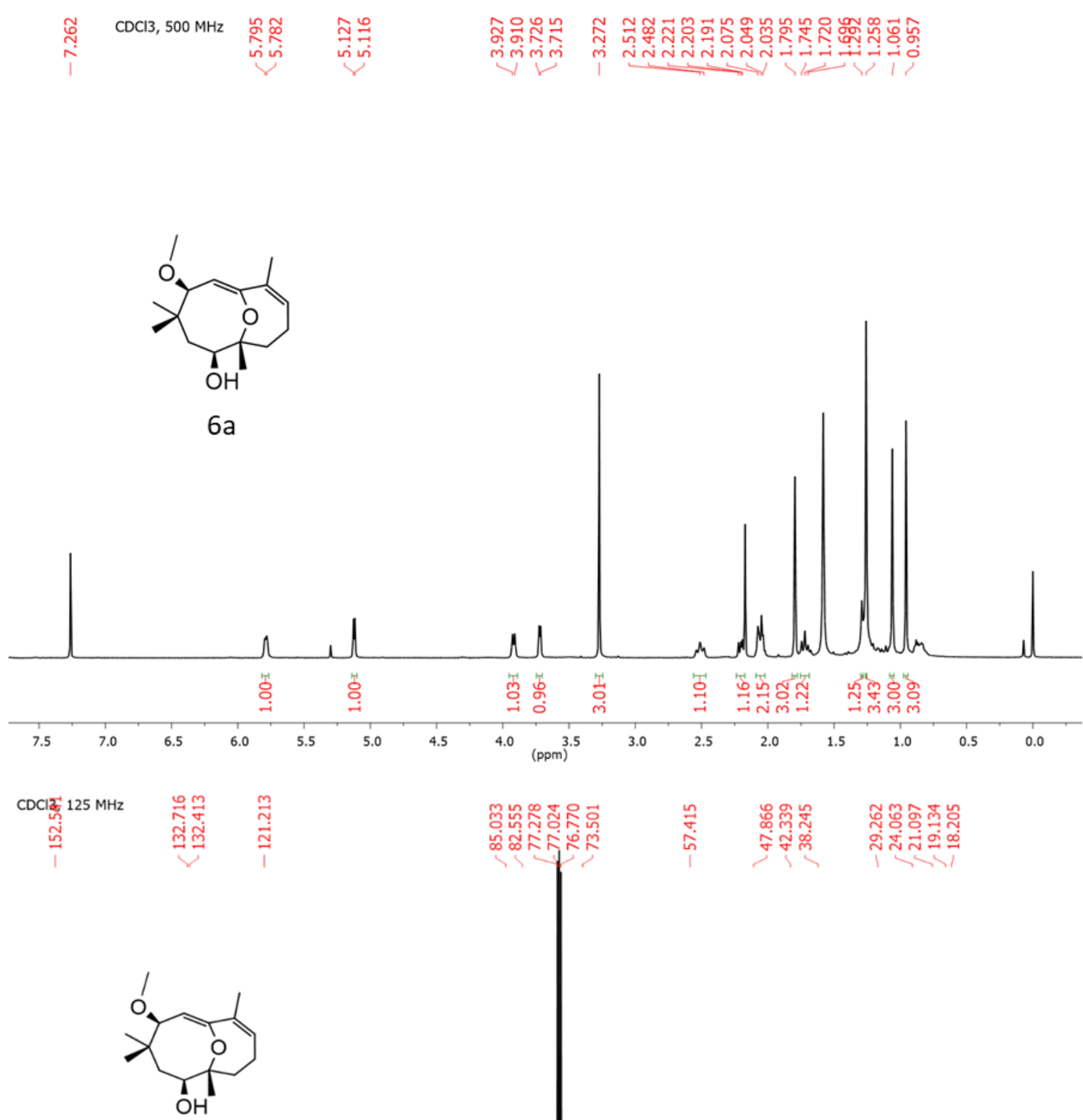

$6 a$
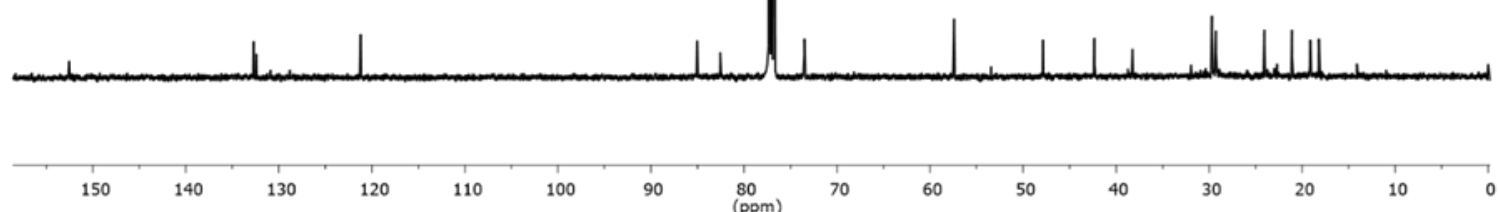


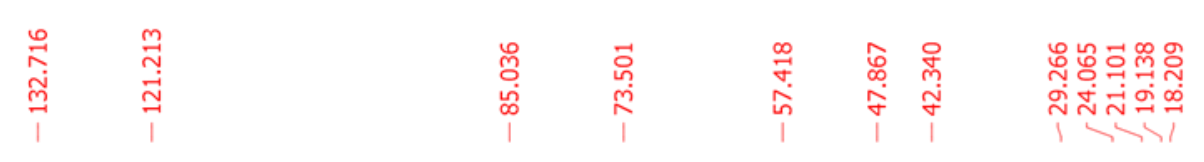
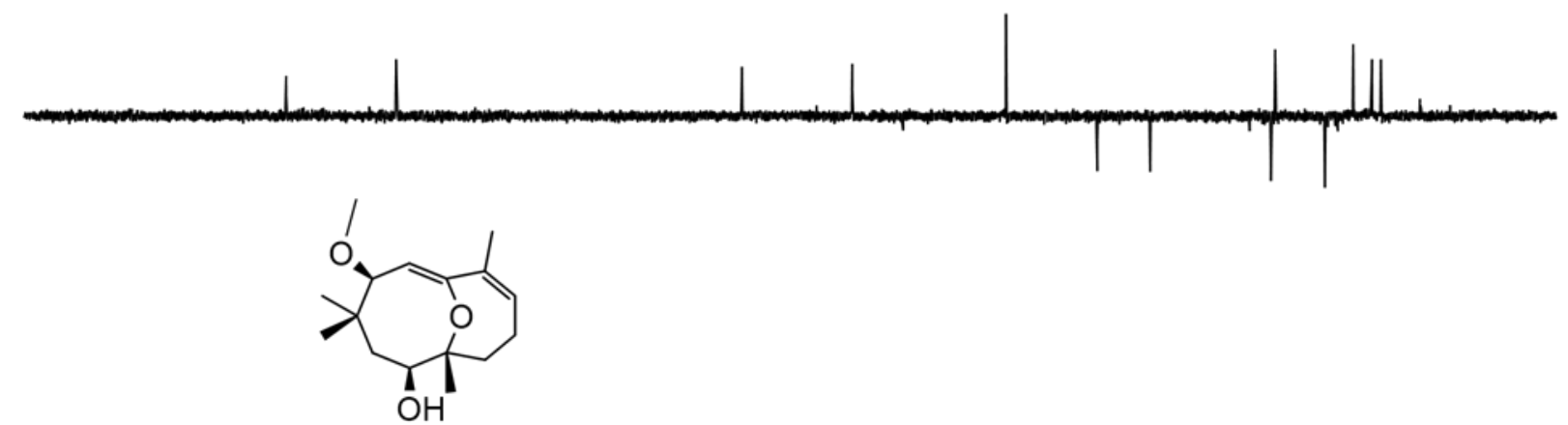

$6 a$
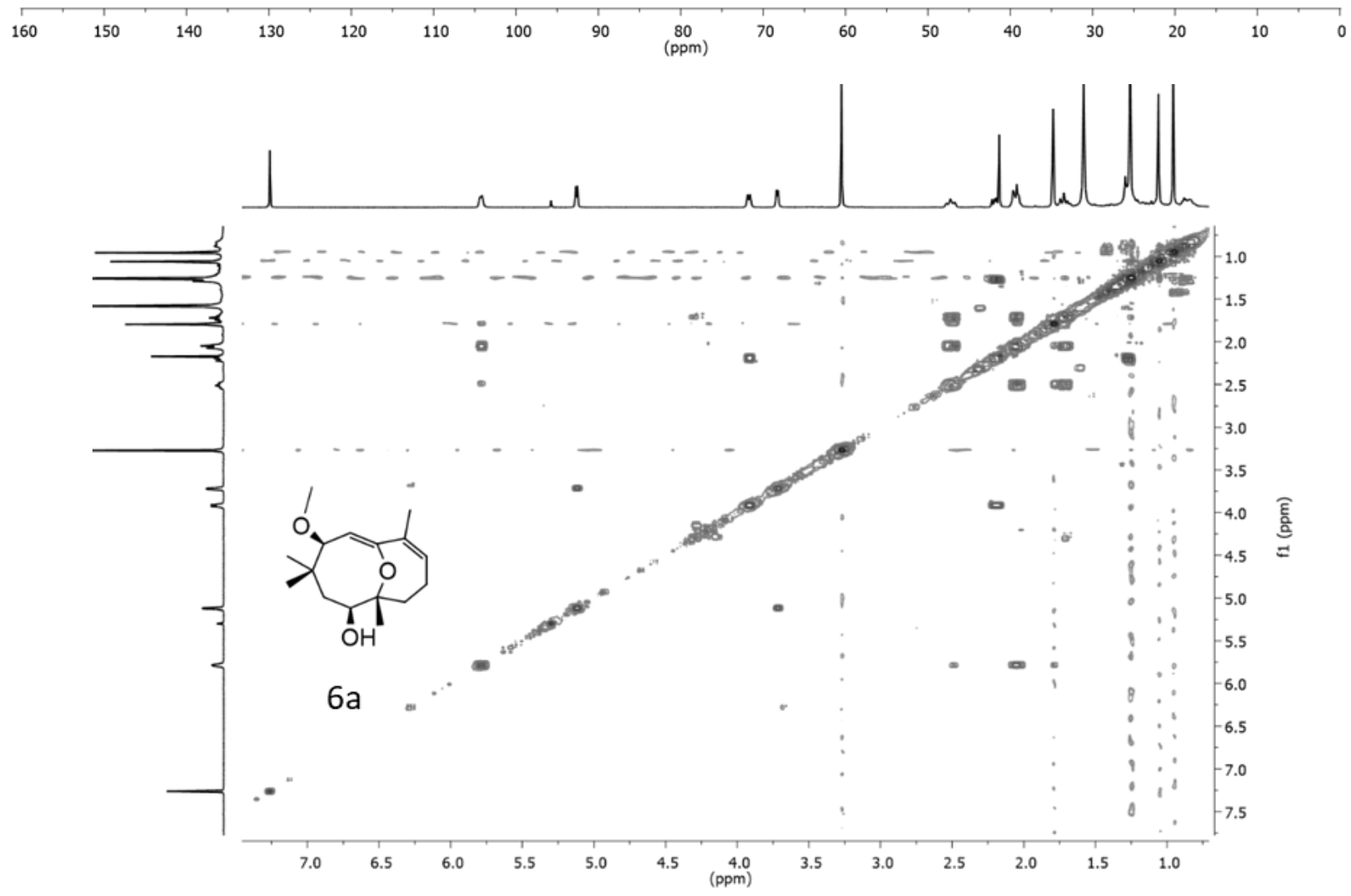


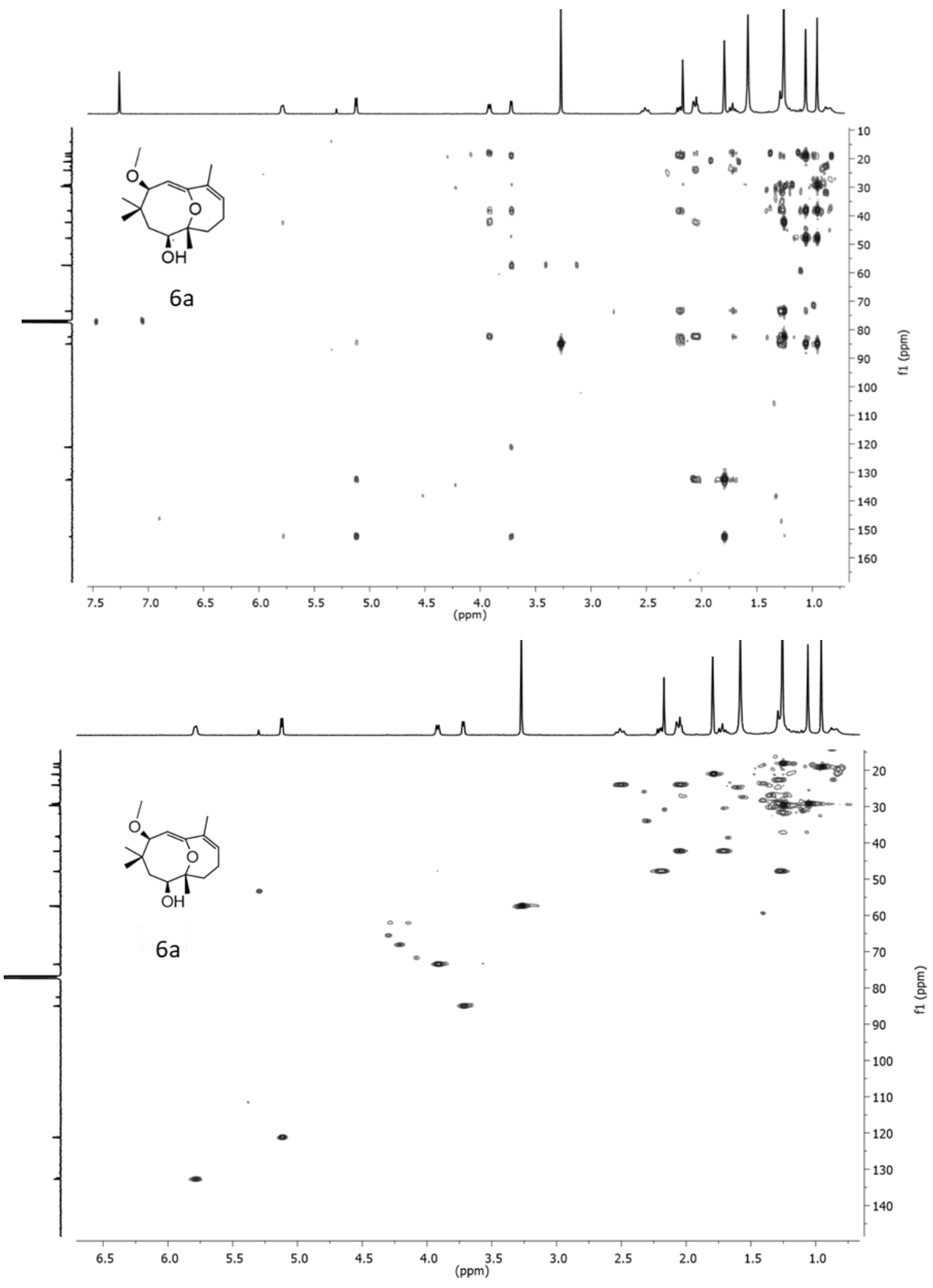


Figure S19. NMR spectra of compound $\mathbf{6 b}$
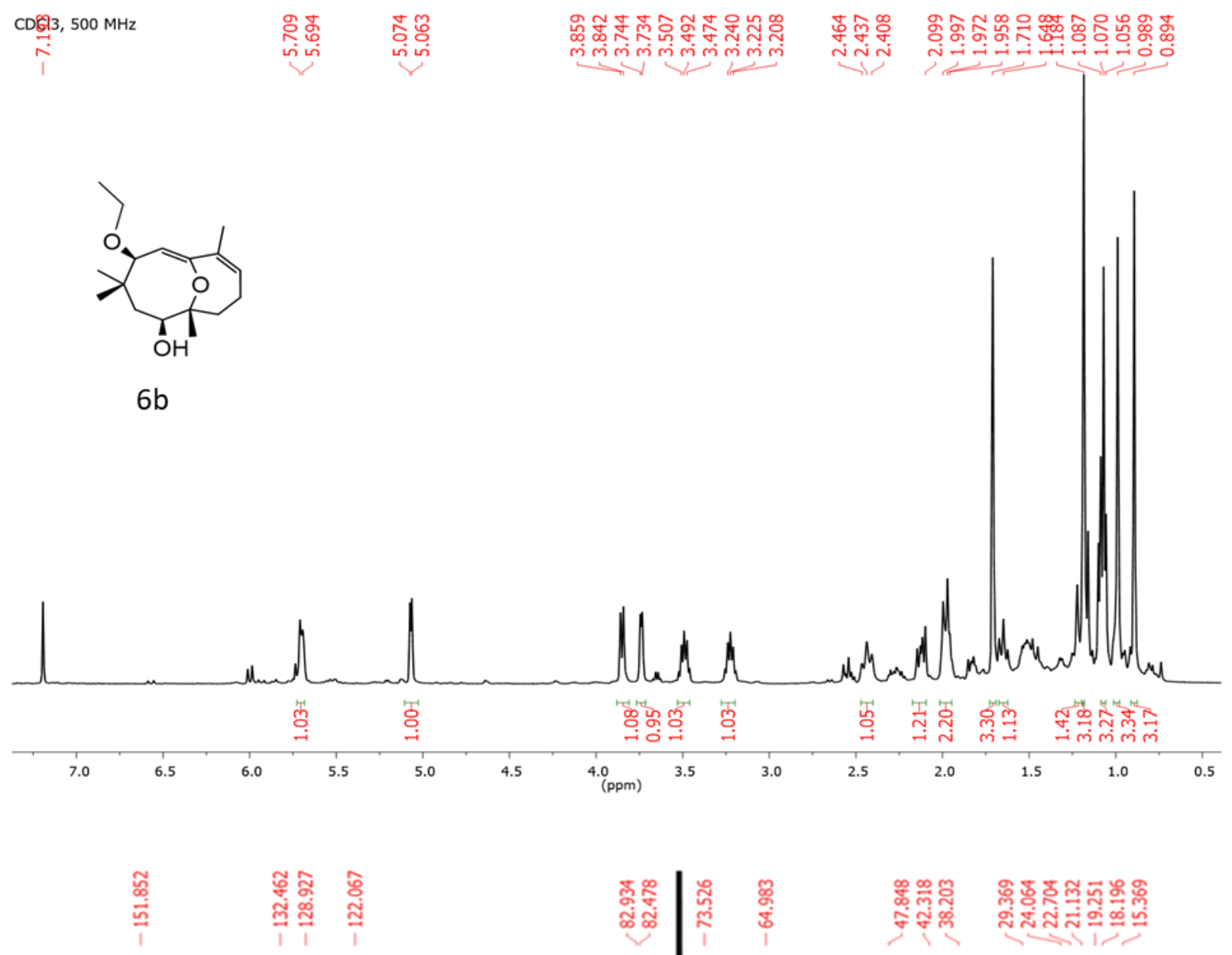

$\mathrm{CDC13}, 125 \mathrm{MHz}$

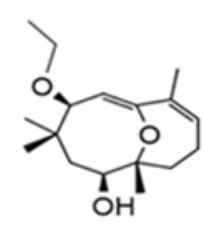

$6 b$
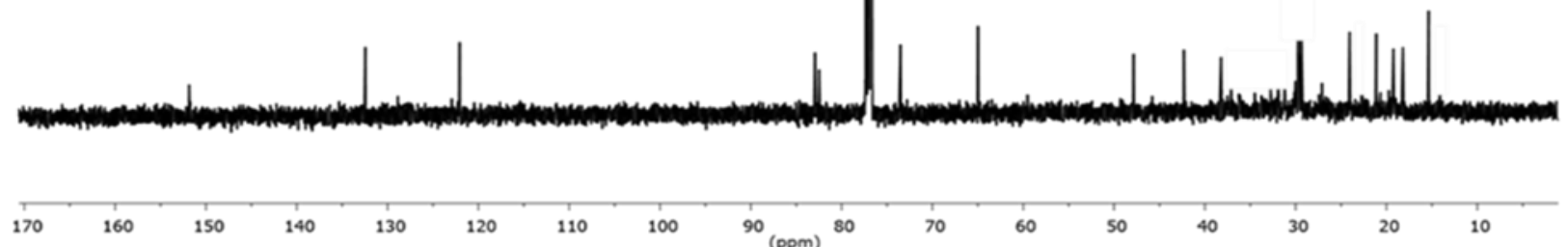


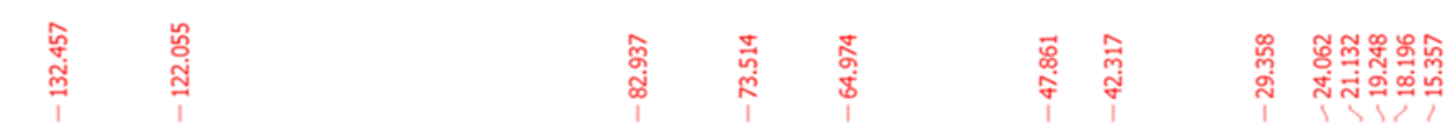
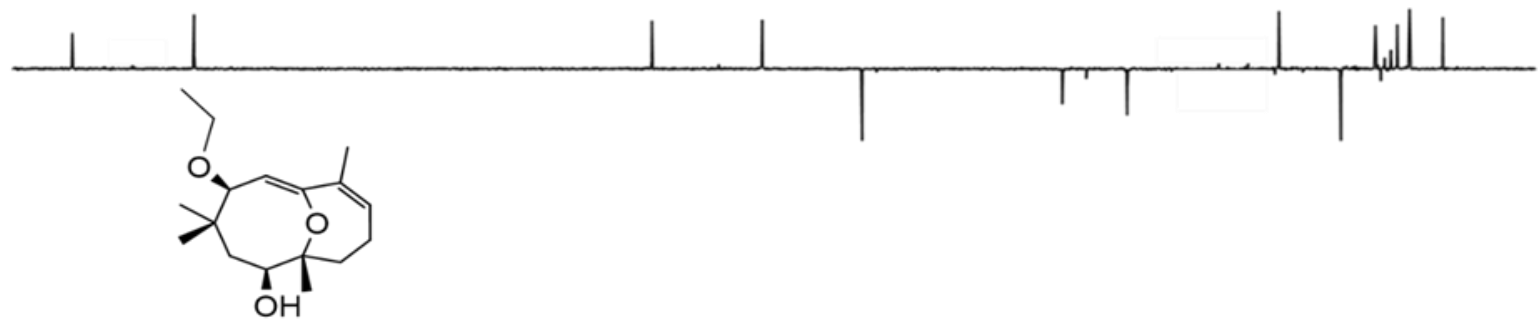

$6 b$

$\begin{array}{llllllllllllllllllllllllllllllll}135 & 130 & 125 & 120 & 115 & 110 & 105 & 100 & 95 & 90 & 85 & 80 & 75 & 70 & 65 & 60 & 55 & 50 & 45 & 40 & 35 & 30 & 25 & 20 & 15 & 10\end{array}$

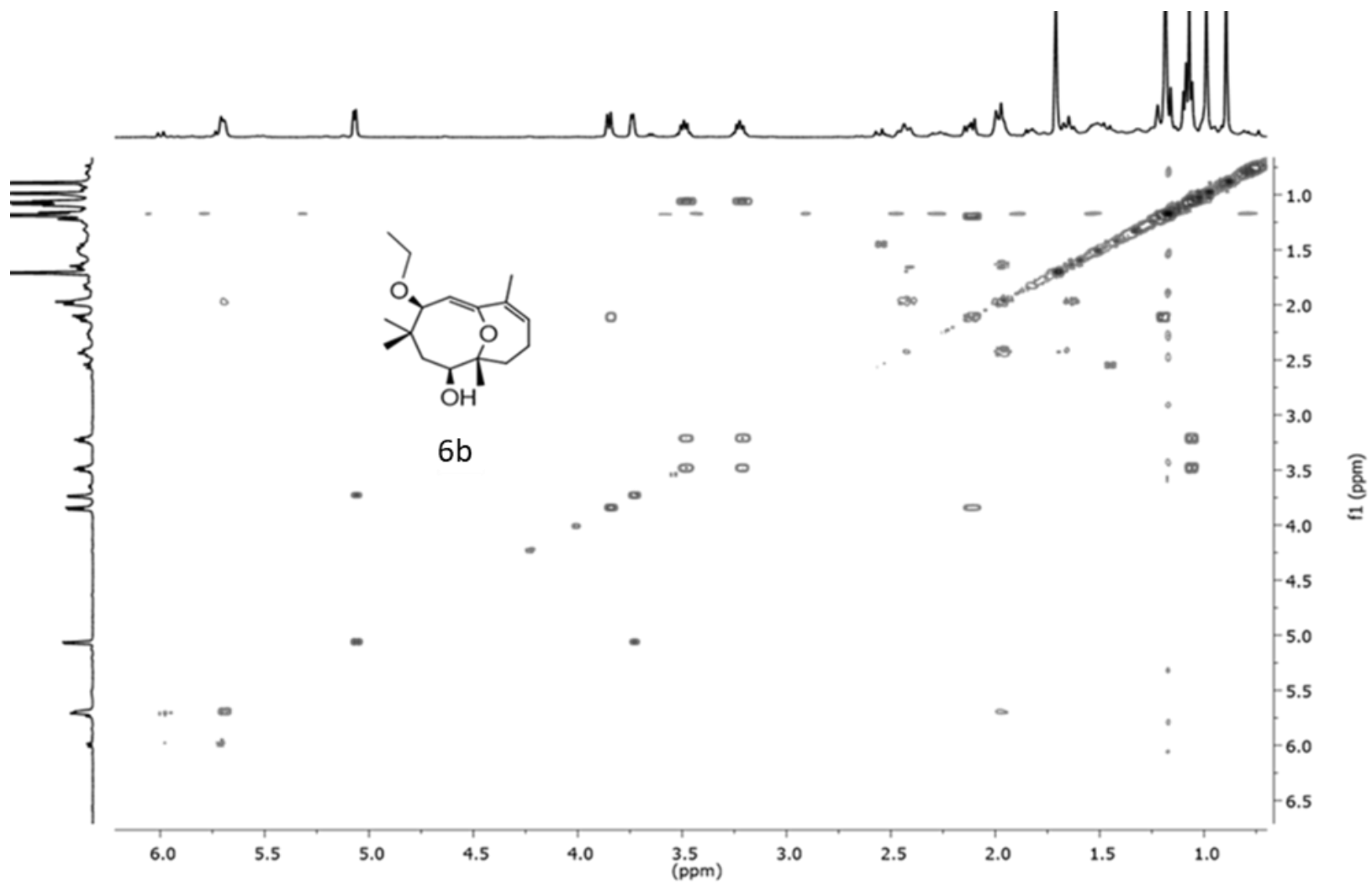



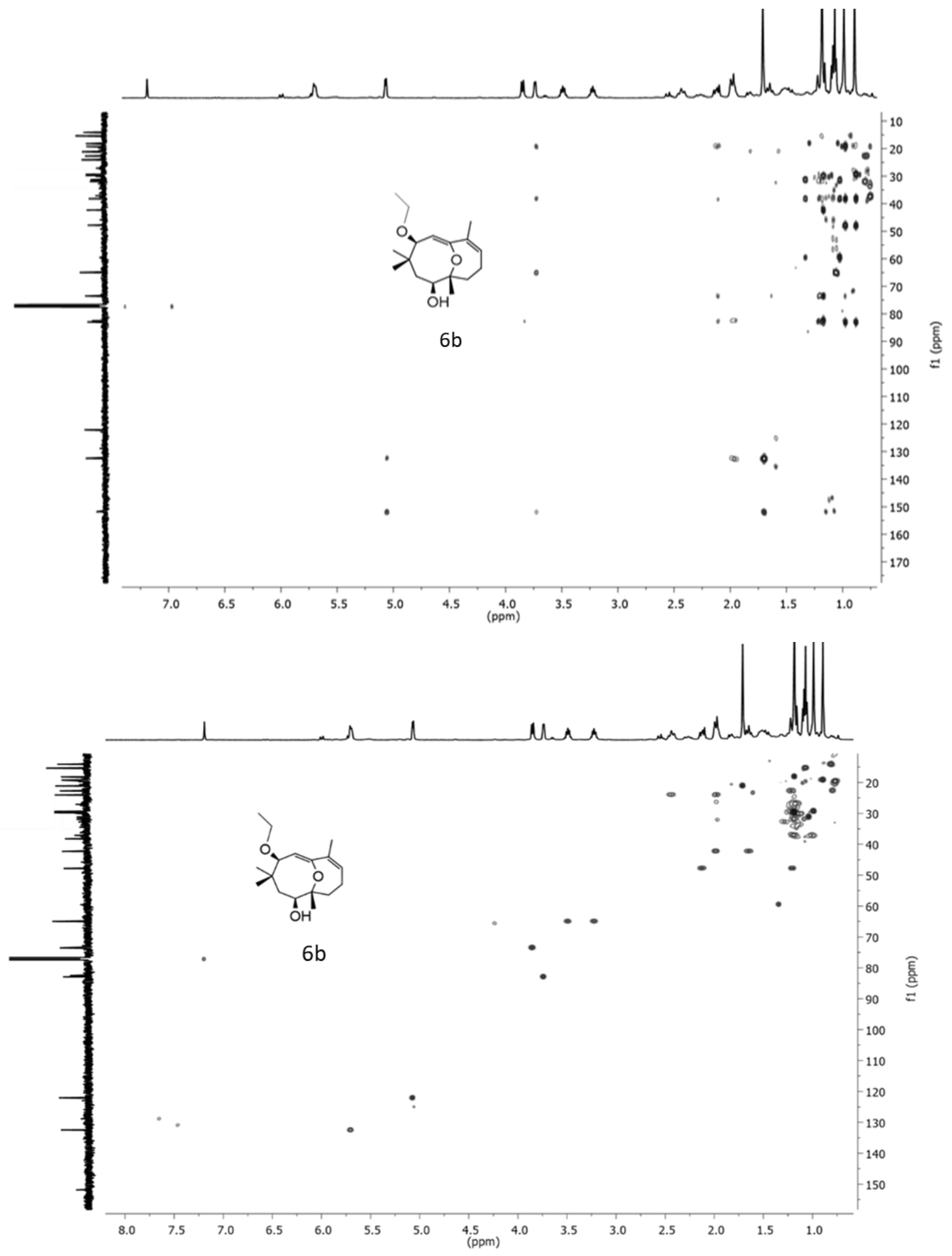